\title{
Modelos flexíveis para dados de tempo de vida em um cenário de riscos competitivos e mecanismos de ativacão latentes
}

\author{
José Julio Flores Delgado \\ Orientador: Prof. Dr. Vicente Garibay Cancho
}

Tese apresentada ao Instituto de Ciências Matemáticas e de Computação - ICMC-USP, como parte dos requisitos para obtenção do título de Doutor em Ciências - Ciências de Computação e Matemática Computacional. VERSÃO REVISADA 
Ficha catalográfica elaborada pela Biblioteca Prof. Achille Bassi e Seção Técnica de Informática, ICMC/USP, com os dados fornecidos pelo(a) autor(a)

\begin{tabular}{|c|c|}
\hline \multirow[t]{3}{*}{$\begin{array}{l}\mathrm{FF} 1063 \\
4 \mathrm{~m}\end{array}$} & $\begin{array}{l}\text { Flores Delgado, José } \\
\quad \text { Modelos flexíveis para dados de tempos de vida em } \\
\text { um cenário de riscos competitivos e mecanismos de } \\
\text { ativaço latentes / José Flores Delgado; orientador } \\
\text { Vicente Garibay Cancho. -- São Carlos, } 2014 \text {. } \\
\quad 82 \text { p. }\end{array}$ \\
\hline & $\begin{array}{l}\text { Tese (Doutorado - Programa de Pós-Graduação em } \\
\text { Ciências de Computação e Matemática Computacional) -- } \\
\text { Instituto de Ciências Matemáticas e de Computação, } \\
\text { Universidade de São Paulo, } 2014 \text {. }\end{array}$ \\
\hline & $\begin{array}{l}\text { 1. Modelo de longa duração. 2. Fatores de risco } \\
\text { latentes. 3. Esquemas de ativação. I. Garibay } \\
\text { Cancho, Vicente, orient. II. Título. }\end{array}$ \\
\hline
\end{tabular}




\section{Agradecimentos}

Agradeço à Pontificia Universidad Católica del Perú, PUCP, por el apoio para o desenvolvimento do curso de doutorado e do trabalho de pesquisa.

Agradeço ao Instituto de Ciências Matemáticas e de Computação, ICMC, por ter usufruído da infraestrutura durante tudo o doutorado. A tudo o pessoal administrativo vai meu especial agradecimento pela atenção recebida.

Agradeço ao professor Vicente Garibay Cancho, pela confiança en mim para iniciar o doutorado e pela dedicada orientação durante tudo o período de estudos.

Agradeço ao professor Mário de Castro, pelas inúmeras consultas recebidas de diferentes temas de Estatística que contribuíram fortemente na conclusão deste doutorado.

Aos professores Marinho Gomes de Andrade e Ricardo Ehlers, por seus magníficos ensinamentos de Docência e Pesquisa.

Ao professor Josemar Rodrigues, quem motivou-me para trabalhar nesta área.

Ao professor Francisco Louzada Neto, pelas sugestões durante o trabalho de pesquisa.

Ao pessoal da biblioteca e da pos-graduação.

Aos colegas alunos da estatística, pelo companheirismo e amizade recebidas deles.

Ao professor Heleno Bolfarine por sempre ter-me motivado a continuar com minha labor de pesquisa.

Ao meu professor do bacharelado e colega Ramón García-Cobián, por ter contribuído fortemente na minha formação acadêmica e moral.

Ao meu colega Arturo Calderón, pelos ensinamentos e amizade recebidos, desde a graduação, que com certeza têm sido úteis também durante tudo o doutorado.

A minha família, pães, irmãos e sogra, pelo apoio recebido durante os estudos.

A realização deste doutorado tem requerido de muita dedicação e esforço pessoal, tarefa que não tivesse conseguido sem a compressão apoio e estimulo da minha querida esposa Silvia. 


\section{Resumo}

Na literatura da área da análise de sobrevivência existem os modelos tradicionais, ou sem fração de cura, e os modelos de longa duração, ou com fração de cura. Recentemente tem sido proposto um modelo mais geral, conhecido como o modelo com fatores de risco latentes com esquemas de ativação. Nesta tese são deduzidas novas propriedades que possuem a função de sobrevivência, a função de taxa de risco e o valor esperado, quando é considerado o modelo com fatores de risco latentes. Estas propriedades são importantes, já que muitos outros modelos que tem aparecido na literatura recentemente podem ser considerados como casos particulares do modelo com fatores de risco latentes. Além disto, são propostos novos modelos de sobrevivência e estes são aplicados a conjuntos de dados reais. Também é realizado um estudo de simulação e uma análise de sensibilidade, para mostrar a qualidade destes modelos. 


\begin{abstract}
In the survival literature we can find traditional models without cure fraction and longterm models with cure fraction. A more general risk factor model with latent activation scheme has been recently proposed. In this thesis we deduce new properties for the survival function, hazard function and expected value for this model. Since many recent survival models can be regarded as particular cases of the risk factor model with latent activation scheme these properties are of great relevance. In addition we propose new survival models that are applied to real data examples. A simulation and sensibility analysis are also performed to asses the goodness of fit of these models.
\end{abstract}




\section{Sumário}

1 Introdução $\quad 15$

1.1 Modelos de sobrevivência com e sem fração de cura . . . . . . . . . . 15

1.2 Organização da tese . . . . . . . . . . . . . . . . . . . . . . . . 18

2 O modelo com fatores latentes $\quad 20$

2.1 Algumas propriedades do modelo . . . . . . . . . . . . . . . 24

2.2 Mecanismo da primeira ativação . . . . . . . . . . . . . . . . . . . 28

2.2.1 Algumas propriedades . . . . . . . . . . . . . . . . . . . . 29

2.2.2 Alguns casos particulares . . . . . . . . . . . . . . . . 31

2.3 Mecanismo da última ativação . . . . . . . . . . . . . . . . . . . . . . 34

2.3.1 Algumas propriedades . . . . . . . . . . . . . . 35

2.3.2 Alguns casos particulares . . . . . . . . . . . . . . . . . 38

2.4 Mecanismo aleatório com distribuição uniforme . . . . . . . . . . . . . 40

3 Uma família de distribuições exponencial-série de potências 42

3.1 Introdução . . . . . . . . . . . . . . . . . . . . . . . . . . 42

3.2 Uma dedução do modelo . . . . . . . . . . . . . . . . . . . . 43

3.3 Algumas propriedades do modelo . . . . . . . . . . . . . . . . . . . . . 44

3.4 Casos particulares . . . . . . . . . . . . . . . . . . . . 47

3.5 Inferência . . . . . . . . . . . . . . . . . . . 50

3.5.1 Estimação por máxima verossimilhança . . . . . . . . . . . . 50 
3.5.2 Estimação por intervalos . . . . . . . . . . . . . . . . 5 50

3.6 Estudo de simulação . . . . . . . . . . . . . . . . . . . . . . . . 51

3.7 Aplicação . . . . . . . . . . . . . . . . . . . . 52

4 Um modelo com fração de cura: exponencial generalizado-série de $\begin{array}{ll}\text { potências com covariáveis } & 56\end{array}$

4.1 Dedução do modelo . . . . . . . . . . . . . . . . . . 56

4.2 Inferência . . . . . . . . . . . . . . . . . . . . . . . . . . . . 59

4.3 Análise de sensibilidade . . . . . . . . . . . . . . . 60

4.3 .1 Influência global . . . . . . . . . . . . . . . 60

4.3 .2 Influência local . . . . . . . . . . . . . . . . . 62

4.4 Aplicação . . . . . . . . . . . . . . . . . . 65

4.4.1 Ajuste de modelos e estimativas dos parâmetros . . . . . . . . 66

4.4 Análise de resíduos . . . . . . . . . . . . . . . 67

4.4 .3 Análise de sensibilidade . . . . . . . . . . . . . . 68

4.4.4 Modelo final e bondade de ajuste . . . . . . . . . . . . . 73

5 Conclusões e pesquisas futuras $\quad 74$

5.1 Conclusões . . . . . . . . . . . . . . . . . . . 74

5.2 Pesquisas futuras . . . . . . . . . . . . . . . . . . 75 


\section{Lista de Tabelas}

3.1 Alguns casos particulares da distribuição de série de potências. . . . . . . . 43

3.2 Funções de densidade e taxa de risco para alguns casos particulares. . . 47

3.3 Momentos, média, variância e média residual para alguns casos particulares.

3.4 Média das variâncias e covariâncias das EMV, erro quadrático meio das EMV e probabilidades de cobertura dos intervalos do $95 \%$ de confiança para os parâmetros. . . . . . . . . . . . . . . . . . 52

3.5 Valores de $l()$, AIC, estatísticas $\chi^{2}$ e K-S para as distribuições ajustadas. . . 54

3.6 Frequências observadas e esperadas para os modelos ajustados. . . . . . 54

3.7 Resumo das estimativas . . . . . . . . . . . . . . . . . . . . . . 55

4.1 Casos particulares da distribuição de série de potências . . . . . . . . . 56

4.2 Funções de sobrevivência $\left(S_{\mathrm{p}}\right)$ e de densidade $\left(f_{\mathrm{p}}\right)$ para alguns modelos 58

$4.3 A I C$ e $S B C$ para os modelos ajustados. . . . . . . . . . . . . . 66

4.4 Resumo das estimativas dos parâmetros do modelo GELF. . . . . . . . . . 66

4.5 Câmbios relativos nas estimativas (rce) e nos erros padrões estimados (rcse) dos parâmetros . . . . . . . . . . . . . . . . . . . 72

4.6 Estimativas de máxima verossimilhança dos parâmetros do modelo GELF. 73 


\section{Lista de Figuras}

1.1 Modelo de mixtura de Boag (1949). . . . . . . . . . . . . . . 16

3.1 Gráficos das funções de densidade e taxa de risco para alguns casos particulares. 48

3.2 Gráfico TTT plot. . . . . . . . . . . . . . . . . . . . . 53

3.3 Esquerda: densidades das distribuições ajustadas sobrepostas ao histograma dos dados. Direita: funções de sobrevivência ajustadas sobrepostas à curva de Kaplan-Meir. . . . . . . . . . . . . . . . . . . . . . . . . . . . . . 54

3.4 Histogramas das réplicas bootstrap. Direita: de $\hat{\theta}$. Esquerda: de $\hat{\lambda}$. . . . . 55

4.1 Estimação de Kaplan-Meier da função de sobrevivência. . . . . . . . . . . . . . 65

4.2 Gráficas de resíduos. Quadro esquerdo: Gráfico de índices dos quantis normalizados aleatorizados do modelo ajustado. Quadro direito: Gráfico QQ dos quantis normalizados aleatorizados e envelope gerado. . . . . . . . . . .

4.3 Gráfico de índices das medidas case-deletion. Quadro esquerdo: A distância generalizada de Cook $\left(G C_{i}\right)$. Quadro direito: A distância da verossimilhança $\left(L D_{i}\right) \ldots \ldots \ldots \ldots \ldots \ldots \ldots \ldots \ldots$

4.4 Gráfico de índices das medidas case-deletion para os sub-vetores de parâmetros. Quadro esquerdo: A distância generalizada de Cook $G C_{i}(\gamma)$. Quadro direito: A distância generalizada de $\operatorname{Cook} G C_{i}(\boldsymbol{\beta})$. . . . . . . . .

4.5 Gráficos de índices das medidas de influência segundo o esquema de perturbação ponderada de casos. Quadro esquerdo: Direção da máxima curvatura, $\boldsymbol{h}_{\max }$. Quadro direito: $M(0)_{i} \ldots \ldots$. . . . . . . . . 70

4.6 Gráficos de índices das medidas de influência segundo o esquema de perturbação ponderada nas respostas. Quadro esquerdo: Direção da máxima curvatura, $\boldsymbol{h}_{\max }$. Quadro direito: $M(0)_{i}$. . . . . . . . . . 
4.7 Gráficos de índices das medidas de influência local segundo o esquema de perturbação para a co-variável grossura.Quadro esquerdo: Direção da máxima curvatura, $\boldsymbol{h}_{\max }$. Quadro direito: $M(0)_{i} \ldots \ldots \ldots 71$

4.8 Gráficos de índices das medidas de influência local segundo o esquema de perturbação ponderada. Quadro esquerdo: Direção da máxima curvatura, $\boldsymbol{h}_{\text {max }}$. Quadro direito: $M(0)_{i}$. . . . . . . . . . . . . 71

4.9 Estimativa de Kaplan-Meier da função de sobrevivência. . . . . . . . . . . 73 


\section{Capítulo 1}

\section{Introdução}

Neste capítulo é introduzido o contexto no qual será desenvolvido o projeto de pesquisa dentro da área da Análise de Sobrevivência.

\subsection{Modelos de sobrevivência com e sem fração de cura}

Análise de sobrevivência é o termo utilizado para designar a análise estatística de dados quando a variável em estudo, $T$, representa o tempo a partir de um instante inicial até a ocorrência de determinado evento de interesse (tempo geralmente denominado de falha).

Os modelos de sobrevivência sem fração de cura ou tradicionais assumem que o evento de interesse necessariamente ocorrerá, ou seja, as unidades da população em que é observada a variável $T$ estão em risco com probabilidade um, assim $P(T=\infty)=0$, como ocorre com toda variável aleatória.

Um dos modelos paramétricos tradicionais nesta situação é o de Weibull, cuja função de distribuição cumulativa está dada por

$$
F(t)=1-e^{-\beta t^{\alpha}}, t>0
$$

onde $\alpha>0$ e $\beta>0$ são os parâmetros do modelo. Uma justificativa para o uso deste modelo é dada em Pike (1966) para explicar o tempo até a ocorrência do câncer em um tecido celular. Aqui é assumido que em cada uma das $m$ células do tecido o câncer pode ocorrer independentemente e com uma mesma função de distribuição cumulativa $G$ e que o menor destes tempos determina a ocorrência do câncer no tecido celular. Uma vez 
que o número $m$ é muito grande, uma distribuição assintótica do valor mínimo, como a distribuição Weibull, pode ser usada para describer o tempo até a ocorrência do câncer no tecido. Que a distribuição de Weibull sirva para modelar taxas de risco constantes e monótonas (crescentes e decrescentes) é uma das suas propriedades principais.

Por outra parte, existem situações em que uma proporção de unidades da população não são suscetíveis ao evento de interesse, assim $P(T=\infty)>0$ e, por conseguinte, $T$ deve ser uma variável aleatória estendida (Ash, 1972, p. 209). Por exemplo, se o evento de interesse é a recorrência de certo tipo de câncer após aplicação de tratamentos, então uma proporção dos indivíduos em estudo pode não apresentar o retorno da doença. Nesta situação os modelos de sobrevivência tradicionais podem ser inadequados; assim, tem-se desenvolvido modelos que consideram esta possibilidade, conhecidos na literatura como modelos com fração de cura ou de longa duração. Uma maneira de reconhecer que os dados apresentam esta característica é através da estimativa da função de sobrevivência de Kaplan-Meier (Kaplan \& Meier, 1958), pois esta apresenta uma cauda direita em um nível aproximadamente constante e estritamente maior do que zero por um período de tempo considerável. A abordagem clássica para os estudos dos dados de sobrevivência com fração de cura é o modelo de mistura proposto por Boag (1949). Neste modelo é assumido que a população de unidades de estudo está formada por unidades imunes ao evento de interesse (ou curados) e suscetíveis (ou não curados), como é representado na Figura 1.1 (extraida do artigo de Boag (1949)).

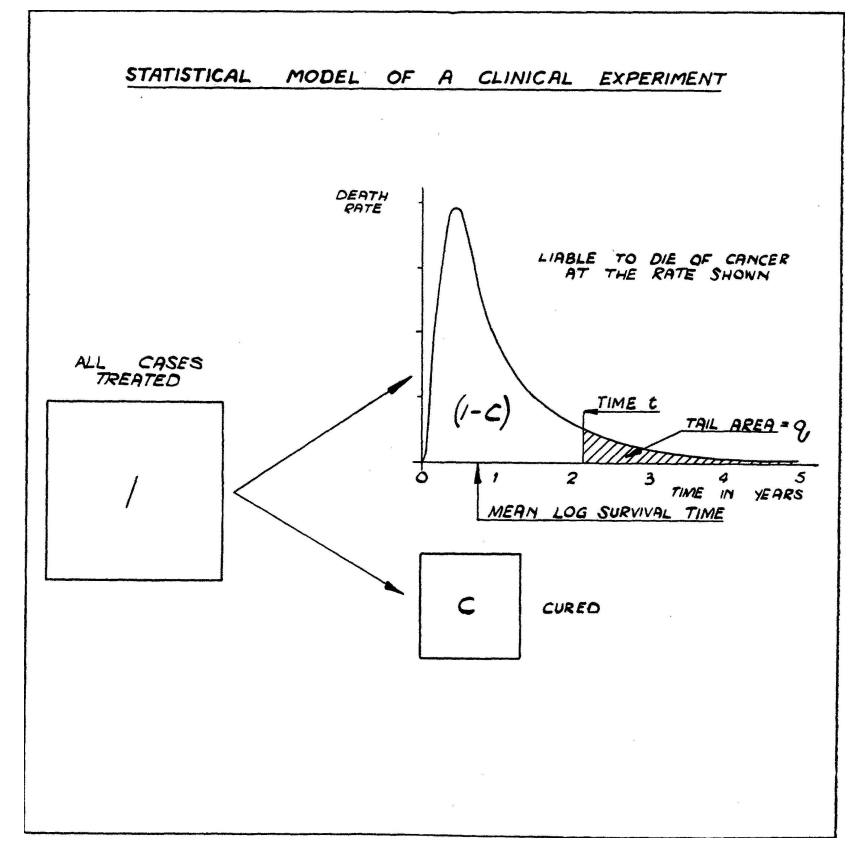

Figura 1.1: Modelo de mixtura de Boag (1949). 
Este modelo tem sido estudado por muitos autores, por exemplo en Berkson \& Gage (1952) a função de sobrevivência, denotada por $S_{p}$, é dada por

$$
S_{p}(t)=p_{0}+\left(1-p_{0}\right) S(t),
$$

em que $S(\cdot)$ representa a função de sobrevivência dos indivíduos não curados (ou, mais geralmente, das unidades suscetíveis ao evento de interesse) e $p_{0}=\lim _{t \rightarrow \infty} S_{p}(t)$ representa a fração de curados (Boag, 1949) (ou das unidades não suscetíveis ao evento de interesse). $S_{p}(\cdot)$ é uma função de sobrevivência estendida, $\lim _{t \rightarrow \infty} S_{p}(t)>0$, e corresponde a mistura de uma variável aleatória $Z$, com função de sobrevivência $S(\cdot)$, e o valor infinito:

$$
T= \begin{cases}\infty, & \text { se } M=0, \\ Z, & \text { se } M=1,\end{cases}
$$

em que $M$ é uma variável aleatória independente de $Z$ e distribuição de Bernoulli com $P(M=0)=p_{0}$.

Outras propostas para distribuição dos indivíduos suscetíveis ao evento de interesse como, por exemplo, Cancho \& Bolfarine (2001), que propõem o modelo de mistura Weibull exponenciada. Farewell (1977) assume que a fração de cura para a $i$-ésima unidade na amostra de tamanho $n$ é dada por uma regressão logística na qual $p_{0}$ está relacionada com as covariáveis por

$$
p_{0 i}=\frac{\exp \left(\boldsymbol{x}_{i}^{\top} \boldsymbol{\beta}\right)}{1+\exp \left(\boldsymbol{x}_{i}^{\top} \boldsymbol{\beta}\right)}, i=1,2, \ldots, n,
$$

em que $\boldsymbol{x}_{i}=\left(x_{i 1}, \ldots, x_{i k}\right)^{\top}$ é o vetor dos valores correspondentes às covariáveis e $\boldsymbol{\beta}=\left(\beta_{1}, \ldots, \beta_{k}\right)^{\top}$ é o vetor de coeficientes das covariáveis.

Maller \& Zhou (1992) propõe um estimador não paramétrico para a fração de cura.

Modelos de sobrevivência com fração de cura alternativos foram propostos e estudados por Yakovlev \& Tsodikov (1993), Tsodikov (1998) e Chen et al. (1999). Estes modelos, que serão chamados neste trabalho de tempo de promoção (como referido em de Castro et al. (2010)), têm uma motivação biológica e assumem a existência de fatores de risco latentes e a ativação de um deles origina o evento de interesse. É assumido que o número de fatores de risco segue uma distribuição de Poisson com parâmetro $\theta$ e que os tempos de ativação são independentes e independentes no número de fatores de risco e com função de sobrevivência $S_{a}$. Assim a função de sobrevivência da população é

$$
S_{p}(t)=\exp \left\{-\theta\left(1-S_{a}(t)\right)\right\},
$$

A função de sobrevivência em (1.4) tem a forma da corresponde ao modelo de mistura dado em (1.1)

$$
S_{p}(t)=p_{0}+\left(1-p_{0}\right) S(t),
$$


em que $p_{0}=\lim _{t \rightarrow \infty} S_{p}(t)=e^{-\theta}$ e $S(t)=\frac{\exp \left\{-\theta\left(1-S_{a}(t)\right)\right\}-p_{0}}{1-p_{0}}$.

Quando o parâmetro $\theta$ é relacionado com as covariáveis por

$$
\theta_{i}=\exp \left(\boldsymbol{x}_{i}^{\top} \boldsymbol{\beta}\right)
$$

como em Tsodikov (1998) e Chen et al. (1999), o modelo apresenta a propriedade de riscos proporcionais, propriedade que não possui o modelo de mistura.

O modelo de promoção tem sido estudado e desenvolvido intensamente na literatura. Hoggart \& Griffin (2001) desenvolveu um modelo para estudar o tempo até que um indivíduo deixe de ser cliente de um banco. Chi \& Ibrahim (2007) propõem um modelo para dados longitudinais e de sobrevivência, em que a fração de cura pode ser nula ou positiva. Rodrigues et al. (2009b) discutem o uso da distribuição Conway-Maxwell Poisson na estimação da proporção de curados. Cancho et al. (2011b) consideram um modelo com fração de cura baseado na distribuição binomial negativa.

Entre outras referências básicas para os modelos com fração de cura estão os livros de Maller \& Zhou (1996) e Ibrahim et al. (2001).

Na literatura, além dos modelos de sobrevivência tradicionais (sem fração de cura), existem os modelos de fração de cura (ou longa duração) e entre estes últimos estão os de mistura e os de tempo de promoção. Recentemente, tem-se desenvolvido trabalhos que permitem unificar a teoria para os modelos de longa duração, como em Cooner et al. (2007), Rodrigues et al. (2009a) e Kim et al. (2011). Embora não seja mencionado nestes trabalhos, também é possível obter modelos tradicionais (sem fração de cura) a partir destes.

Como o estudo trata sobre os dois tipos de modelos de sobrevivência mencionados (tradicionais e de longa duração), no seguinte capítulo será apresentada uma teoria que permite unificá-los; esta teoria proporcionará uma estrutura geral para desenvolver o trabalho com os dois tipos de modelos de sobrevivência.

\subsection{Organização da tese}

O estudo está organizada como segue:

No Capítulo 2 é apresentada uma teoria unificada de modelos de sobrevivência que considera fatores ou causas de risco latentes do evento de interesse. Esta teoria, que será chamada de modelos com fatores de risco latentes, foi proposta por Cooner et al. (2007). São apresentados novos resultados a respeito. Como exemplos de ilustração da 
teoria tratada, são deduzidos alguns modelos já existentes na literatura que foram propostos antes desta teoria, por tanto algumas propriedades destes modelos, que foram justificadas em cada caso particular, são explicadas pelos novos resultados apresentados neste estudo. Também são deduzidas algumas funções de sobrevivência que aparentemente não tem referências na literatura e são propostas para pequisas futuras.

No Capítulo 3 é utilizada a teoria dos modelos com fatores latentes para deduzir uma nova família de distribuições. São estudas as propriedades da família e feita uma aplicação. A maior parte dos resultados conduziram ao artigo de Flores Delgado et al. (2013).

No Capítulo 4 a teoria dos modelos com fatores latentes é utilizada para deduzir um modelo com fração de cura com covariáveis. Também é resumida a teoria para realizar uma análise de sensibilidade e feita uma aplicação para um conjunto de dados reais. A maior parte dos resultados conduziram ao relatório Flores Delgado et al. (2012) e a um artigo submetido.

As conclusões são dadas no Capítulo 5. 


\section{Capítulo 2}

\section{O modelo com fatores latentes}

Os modelos com fatores ou riscos latentes propõem um mecanismo para explicar como é originada a variável aleatória $T$, que representa o tempo até a ocorrência do evento de interesse, na presença da fração de cura como na ausência dela, resultando um modelo unificador. A teoria a seguir esta baseada no artigo de Cooner et al. (2007) e usa idéias do artigo de Rodrigues et al. (2009a). Alguns resultados novos para este modelo são deduzidos. Esta teoria generaliza os trabalhos de Chen et al. (1999) e Yakovlev et al. (1993). O modelo com fatores (de risco) latentes, Cooner et al. (2007), fica definido pelas suposições seguintes:

$\left(\mathrm{s}_{1}\right)$ Seja $M$ uma variável aleatória discreta, com valores no conjunto $\{0,1, \ldots\}$ e cuja função de probabilidades $f_{M}(\cdot)$ é tal que $P(M=0)<1$. $M$ representa o número de causas ou fatores de risco latentes que levariam a ocorrência do evento de interesse. Denota-se $p_{0}=P(M=0)$.

$\left(\mathrm{s}_{2}\right)$ Seja $K$ uma variável aleatória discreta, com valores no conjunto $\{0,1, \ldots\}$ e com função de probabilidades condicional, dado $M=m, f_{K \mid M=m}$ tal que $P(1 \leq K \leq m \mid M=m)=1$, para $m \in\{1,2, \ldots\}$. Se tiver fatores de risco, a variável $K$ representa o número mínimo que devem-se ativar para que ocorra o evento de interesse. Se não tiver fatores de risco, ou seja se $M=0, K$ pode definir-se arbitrariamente.

$\left(\mathrm{s}_{3}\right)$ Tem-se a sequência de variáveis aleatórias continuas independentes, $Z_{1}, Z_{2}, \ldots$, com a mesma função de sobrevivência, $S_{a}(\cdot)$, e independentes de $M$ e $K$. Esta sequência de variáveis representam os tempos de ocorrência ou de ativação dos fatores latentes. 
$\left(\mathrm{s}_{4}\right)$ O tempo até a ocorrência do evento de interesse é a variável aleatória estendida $T$ definida por

$$
T= \begin{cases}\infty, & \text { se } M=0 \\ Z_{(K, M)}, & \text { se } M \geq 1\end{cases}
$$

onde, $Z_{(1, M)} \leq \cdots \leq Z_{(K, M)} \leq \cdots \leq Z_{(M, M)}$ são as estatísticas de ordem das variáveis aleatórias $Z_{1}, \ldots, Z_{M}$. Assim, se $M=0$, ou seja, se não existem fatores de risco, $T=\infty$ (isto justificará a não ocorrência do evento de interesse e conseqüente fração de cura); se $M \geq 1$, ou seja, se existe pelo menos um fator de risco latente, assuma-se que $K \operatorname{dos} M$ fatores de risco devem-se ativar para produzir a ocorrência do evento de interesse.

A função de sobrevivência de $T$ será denotada por $S_{p}(\cdot)$ (como no Capítulo 1) e uma expressão para esta é dada no teorema seguinte.

Teorema 2.1. Sob as suposições $\left(s_{1}\right)-\left(s_{4}\right)$ a função de sobrevivência de T é dada por

$$
S_{p}(t)=p_{0}+\left(1-p_{0}\right) S^{*}(t), \text { para } t>0
$$

em que

$$
S^{*}(t)=\frac{1}{1-p_{0}} \sum_{m=1}^{\infty} \sum_{k=1}^{m} \sum_{j=m-k+1}^{m}\left(\begin{array}{c}
m \\
j
\end{array}\right) S_{a}^{j}(t)\left[1-S_{a}(t)\right]^{m-j} f_{K \mid M}(k){ }_{M=m} f_{M}(m) .
$$

Além disso, a série de funções em t, dada em (2.3), converge uniformemente. Também $S^{*}(\cdot)$ satisfaz as propriedades usuais de uma função de sobrevivência associada com uma variável aleatória continua positiva, isto é,

i) $S^{*}(\cdot)$ é uma função continua,

ii) $S^{*}(\cdot)$ é uma função decrescente,

iii) $\lim _{t \rightarrow 0^{+}} S^{*}(t)=1 e \lim _{t \rightarrow \infty} S^{*}(t)=0$.

Estas propriedades também são satisfeitas por $S_{p}(\cdot)$, exceto que em iii) $\lim _{t \rightarrow \infty} S_{p}(t)=p_{0}$.

Note-se que $S^{*}(\cdot)$ corresponde à função de sobrevivência das unidades suscetíveis. A Equação (2.3) é equivalente a Equação (2) em Cooner et al. (2007). Vale a pena salientar que esta forma equivalente de expressar $S^{*}(\cdot)$ e a convergência uniforme facilitam o estudo das propriedades de $S^{*}(\cdot)$ e ajuda a gerar novas funções de sobrevivência. 
Prova. Desde que $S_{p}(t)=P(T>t)$, segue que

$$
S_{p}(t)=P(T>t, M=0)+P(T>t, M \geq 1)=P(M=0)+P(T>t, M \geq 1),
$$

onde a última igualdade deve-se a que $\{M=0\}=\{T=\infty\}=\{T>t, M=0\}$.

Por outra parte,

$$
\begin{aligned}
P(T>t, M \geq 1) & =P\left(Z_{(K, M)}>t, M \geq 1\right) \\
& =\sum_{m=1}^{\infty} \sum_{k=1}^{\infty} P\left(Z_{(k, m)}>t, K=k, M=m\right) \\
& =\sum_{m=1}^{\infty} \sum_{k=1}^{m} P\left(Z_{(k, m)}>t \mid M=m, K=k\right) P(K=k \mid M=m) P(M=m) ;
\end{aligned}
$$

Levando em conta, nesta ultima igualdade, que a sequência de variáveis $Z_{i}$ é independente de $K$ e de $M$, seque que

$$
P(T>t, M \geq 1)=\sum_{m=1}^{\infty} \sum_{k=1}^{m} P\left(Z_{(k, m)}>t\right) f_{K \mid M=m}(k) f_{M}(m) .
$$

Aplicando nesta última igualdade o resultado conhecido a respeito das estatísticas de ordem, $Z_{(1, m)} \leq \cdots \leq Z_{(m, m)}$, de uma amostra aleatória de tamanho $m, Z_{1}, \ldots, Z_{m}$, com função de sobrevivência comum $S_{a}(\cdot)$ :

$$
P\left(Z_{(k, m)}>t\right)=\sum_{j=m-k+1}^{m}\left(\begin{array}{c}
m \\
j
\end{array}\right) S_{a}^{j}(t)\left[1-S_{a}^{j}(t)\right]^{m-j},
$$

tem-se

$$
P(T>t, M \geq 1)=\sum_{m=1}^{\infty} \sum_{k=1}^{m} \sum_{j=m-k+1}^{m}\left(\begin{array}{c}
m \\
j
\end{array}\right) S_{a}^{j}(t)\left[1-S_{a}(t)\right]^{m-j} f_{K \mid M}(k) \underset{M}{f} f_{M}(m) .
$$

Assim, (2.3) segue de (2.4) e (2.5).

Para estabelecer a convergência uniforme da série de funções em $t$ dada em (2.3), observe-se que $S^{*}(t)=\sum_{m=1}^{\infty} S_{m}^{*}(t)$, onde

$$
S_{m}^{*}(t)=\frac{1}{1-p_{0}} \sum_{k=1}^{m} \sum_{j=m-k+1}^{m}\left(\begin{array}{c}
m \\
j
\end{array}\right) S_{a}^{j}(t)\left[1-S_{a}(t)\right]^{m-j} f_{K \mid M=m}(k) \underset{M}{f}(m), \quad m \in\{1,2, \ldots\}
$$

mas, $\forall m \in\{1,2, \ldots\}, \forall t>0:\left|S_{m}^{*}(t)\right| \leq \frac{1}{1-p_{0}} f_{M}(m)$, com $\sum_{m=1}^{\infty} \frac{1}{1-p_{0}} f_{M}(m)<\infty$. Então, o resultado segue do Critério de Weierstrass (Rudin, 1976, p. 148).

As propriedades $i$ ) e iii) seguem das propriedades da função de sobrevivência $S_{a}(\cdot)$ e da convergência uniforme demonstrada anteriormente, pois, esta última permite a troca entre limite e série. A propriedade ii) segue imediatamente observando que $S^{*}(t)=\frac{1}{1-p_{0}} P(T>t, M \geq 1)$, segundo (2.3) e (2.5). 
A partir do teorema anterior convém dar as observações seguintes

a) Quando $p_{0}>0$, o modelo com fatores de risco latentes leva a um modelo de mistura cuja fração de cura é dada por $p_{0}=P(M=0)$ e cuja função de sobrevivência das unidades suscetíveis é $S^{*}$ dada em (2.3).

b) Um modelo de mistura, com fração de cura $p_{0}$ e função de sobrevivência das unidades suscetíveis $S$, corresponde a um modelo com fatores de risco latentes, onde o número de fatores latentes $M$ segue uma distribuição de Bernoulli, com $P(M=0)=p_{0}$, e a função de sobrevivência dos tempos de ativação $S_{a}(t)=S(t)$.

c) Se $p_{0}=0$ o modelo de fatores de risco latentes corresponde a um modelo sem fração de cura, com função de sobrevivência $S^{*}$ dada em (2.3).

d) O modelo de tempo de promoção corresponde a um modelo de fatores de risco latentes no qual $M$ segue uma distribuição de Poisson, $K=1$ e os tempos de ativação seguem uma distribuição exponencial.

Portanto, o modelo de fatores de risco latentes de Cooner et al. (2007), além de explicar a origem do tempo até a ocorrência do evento de interesse, $T$, e generalizar os modelos de mistura e de promoção, é unificador, pois, inclui os modelos com e sem fração de cura. Este modelo também proporciona uma maneira geral de obter famílias de funções de sobrevivência, usuais e estendidas. Para isto é suficiente fixar a distribuição do número de fatores latentes, $f_{M}(\cdot)$, a função de sobrevivência dos tempos de ativação, $S_{a}$, e a distribuição condicional do número de fatores que precisam ativar-se para produzir o evento de interesse, dado o número de fatores latentes, $f_{K}(\cdot)_{M}{ }_{=} \underset{=}{\operatorname{Se}} P(M=0)=0$, é obtida uma função de sobrevivência usual. Se $P(M=0)>0$ é obtida uma distribuição de sobrevivência estendida $S_{p}$.

Uma proposta similar ao modelo de Cooner et al. (2007) é dada em Kim et al. (2011), em que $K$ e $M$ são independentes e o tempo $T$ (até a ocorrência do evento) é dado por

$$
T= \begin{cases}\infty, & \text { se } M<K, \\ Z_{(K, M)}, & \text { se } M \geq K,\end{cases}
$$

ou seja, $K$ é um limiar para a ocorrência do evento de interesse. 


\subsection{Algumas propriedades do modelo}

A seguir são apresentados alguns resultados não deduzidos em Cooner et al. (2007). O corolário seguinte estabelece que quando a distribuição de $M$ é truncada em zero a função de sobrevivência obtida, com o modelo de fatores de risco latentes, é aquela que corresponde à função de sobrevivência das unidades suscetíveis, $S^{*}(\cdot)$ dada em (2.3).

Corolário 2.1. Seja $S_{p}(t)=p_{0}+\left(1-p_{0}\right) S^{*}(t)$ o modelo com fração de cura obtido sob as suposições do Teorema 2.1, considerando uma distribuição para $M, f_{M}(\cdot)$, com $p_{0}>0$. Se no lugar desta distribuição de $M$ é considerada sua distribuição truncada em zero; então, resulta um modelo sem fração de cura com função de sobrevivência $S^{*}(t)$.

Prova. Sejam $S_{p 1}(\cdot)$ e $S_{1}^{*}(\cdot)$ as funções de sobrevivência da população e das unidades suscetíveis, respectivamente, obtidas como o modelo de fatores latentes quando é considerada a distribuição $f_{M}(\cdot)$ truncada no zero. Como $P(M=0)=0$ para a distribuição truncada no zero, segue de (2.2) que

$$
S_{p 1}(t)=S_{1}^{*}(t)=\sum_{m=1}^{\infty} \sum_{k=1}^{m} \sum_{j=m-k+1}^{m}\left(\begin{array}{c}
m \\
j
\end{array}\right) S_{a}^{j}(t)\left[1-S_{a}(t)\right]^{m-j} f_{K \mid M=m}(k) \frac{1}{1-p_{0}} f_{M}(m),
$$

onde a última igualdade deve-se a que a função de distribuição truncada no zero de $f_{M}(\cdot)$ é dada por $\frac{1}{1-p_{0}} f_{M}(\cdot)$. Por tanto, o resultado segue de $(2.7)$ e $(2.3)$.

O corolário anterior é esperado pois, a função truncada no zero dos fatores de risco latentes, $M$, corresponde a distribuição de $M$ quando se sabe que $M>0$, ou seja, no caso em que todas as unidades sejam suscetíveis.

O corolário seguinte dá um limitante superior para a função de sobrevivência das unidades suscetíveis a partir da correspondente aos tempos de ativação, da média do número de fatores de risco latentes e da fração de cura.

Corolário 2.2. Para todo $t>0: S^{*}(t) \leq \frac{E(M)}{1-p_{0}} S_{a}(t)$.

Prova. A partir de (2.3) tem-se que

$$
\begin{aligned}
S^{*}(t) & =\frac{1}{1-p_{0}} \sum_{m=1}^{\infty} \sum_{k=1}^{m} \sum_{j=m-k+1}^{m}\left(\begin{array}{c}
m \\
j
\end{array}\right) S_{a}^{j}(t)\left[1-S_{a}(t)\right]^{m-j} f_{K \mid M}(k) f_{M}(m) \\
& \leq \frac{1}{1-p_{0}} \sum_{m=1}^{\infty} \sum_{k=1}^{m} \sum_{j=m-k+1}^{m}\left(\begin{array}{c}
m \\
j
\end{array}\right) S_{a}^{j}(t)\left[1-S_{a}(t)\right]^{m-j} f_{M}(m) .
\end{aligned}
$$


Por outra parte

$$
\sum_{k=1}^{m} \sum_{j=m-k+1}^{m}\left(\begin{array}{c}
m \\
j
\end{array}\right) S_{a}^{j}(t)\left[1-S_{a}(t)\right]^{m-j}=m S_{a}(t),
$$

igualdade que pode-se justificar considerando uma variável aleatória $Y$ com distribuição binomial de parâmetros $m$ (número de ensaios) e $S_{a}(t)$ (probabilidade do sucesso). Com efeito, o lado direito de (2.9) é igual a $E(Y)$ e o lado esquerdo pode ser expressado como $\sum_{k=1}^{m} P(Y \geq m-k+1)=\sum_{j=1}^{m} P(Y \geq j)$. Assim, o resultado segue lembrando que $E(Y)=\sum_{j=0}^{\infty} P(Y>j)=\sum_{j=1}^{m} P(Y \geq j)$, pois $Y$ somente assume valores naturais (vide James (1981, p. 111)).

O corolário segue de (2.8) e (2.9).

Desde que $E(M)=\sum_{j=1}^{\infty} P(M \geq j) \geq P(M \geq 1)=1-p_{0}$, segue que $\frac{E(M)}{1-p_{0}} \geq 1$, por isso o resultado deste corolário não permite estabelecer a ordem estocastica existente entre $S^{*}(\cdot)$ e $S_{a}(\cdot)$, de fato nas sessões 2.2 e 2.3 estabelece-se que, dependendo da distribuição condicional de $K$ dado $M$, as duas situações podem-se apresentar $S^{*} \leq S_{a}$ e $S_{a} \leq S^{*}$.

O corolário seguinte estabelece uma condição para que o momento $r$-ésimo do tempo até a ocorrência do evento de interesse na população das unidades suscetíveis seja finito. Este resultado também não é deduzido em Cooner et al. (2007) nem tem-se achado referências dele na literatura.

Corolário 2.3. Se a média do número de fatores de risco latentes, $M$, e o momento r-ésimo dos tempos de ativação são finitos; então, o momento r-ésimo do tempo até a ocorrência do evento de interesse para a população das unidades suscetiveis também é finito.

Prova. Desde que para toda variável aleatória continua positiva, $T$, com função de sobrevivência $S(\cdot), E\left(T^{r}\right)=r \int_{0}^{\infty} t^{r-1} S(t) d t$, o resultado segue do corolário anterior.

Antes de continuar com outros resultados não deduzidos em Cooner et al. (2007) é obtida uma expressão para a função de densidade correspondente à função de sobrevivência das unidades suscetíveis, $S^{*}(\cdot)$, equivalente a Equação (3) em Cooner et al. (2007). 
Corolário 2.4. Se $f^{*}(\cdot)$ denota a função de densidade correspondente à função de sobrevivência $S^{*}(\cdot)$ em (2.3); então, $\forall t>0$ :

$$
f^{*}(t)=\frac{1}{1-p_{0}} f_{a}(t) \sum_{m=1}^{\infty} \sum_{k=1}^{m} m\left(\begin{array}{c}
m-1 \\
k-1
\end{array}\right) S_{a}^{m-k}(t) F_{a}^{k-1}(t) \underset{K \mid M=m}{f(k)} f_{M}(m),
$$

onde $f_{a}(\cdot)$ e $F_{a}(\cdot)$ denotam as funções de densidade e cumulativa, respectivamente, dos tempos de ativação dos fatores de risco latentes.

Além disso, se $E(M)<\infty$ a série em (2.10), como função de $t$, converge uniformemente.

Prova. O resultado segue de obter a derivada de $S^{*}$ a partir da expressão seguinte:

$$
S^{*}(t)=\frac{1}{1-p_{0}} \sum_{m=1}^{\infty} \sum_{k=1}^{m} m\left(\begin{array}{c}
m-1 \\
k-1
\end{array}\right) \int_{0}^{S_{a}(t)} u^{m-k}(1-u)^{k-1} d u \underset{K \mid M=m}{f(k)} f_{M}(m) .
$$

Esta expressão equivalente para $S^{*}(t)$, que facilita achar sua derivada, pode-se deduzir a partir da igualdade seguinte relacionada com a função beta incompleta (vide Johnson et al. (2005, p. 18)) ou, equivalentemente, com a relação que existe entre a função de distribuição cumulativa da distribuição beta e a distribuição binomial (vide Mendenhall \& Sincich (1995, p. 247)):

$$
\begin{aligned}
\sum_{j=k}^{m}\left(\begin{array}{c}
m \\
j
\end{array}\right) p^{j}(1-p)^{m-j} & =\int_{0}^{p} \frac{\Gamma(m+1)}{\Gamma(k) \Gamma(m-k+1)} u^{k-1}(1-u)^{m-k} d u \\
& =m\left(\begin{array}{c}
m-1 \\
k-1
\end{array}\right) \int_{0}^{p} u^{k-1}(1-u)^{m-k} d u .
\end{aligned}
$$

Para estabelecer a convergência uniforme da série de funções de $t$ em (2.10), observe-se que $\sum_{m=1}^{\infty} \sum_{k=1}^{m} m\left(\begin{array}{c}m-1 \\ k-1\end{array}\right) S_{a}^{m-k}(t) F_{a}^{k-1}(t) \underset{K \mid M=m}{f(k)} f_{M}(m)=\sum_{m=1}^{\infty} f_{m}^{*}(t)$, onde

$$
f_{m}^{*}(t)=\sum_{k=1}^{m} m\left(\begin{array}{c}
m-1 \\
k-1
\end{array}\right) S_{a}^{m-k}(t) F_{a}^{k-1}(t) \underset{K \mid M=m}{f(k)} f_{M}(m),
$$

mas, $\forall m \in\{1,2, \ldots\}, \forall t>0:\left|f_{m}^{*}(t)\right| \leq m f_{M}(m)$, com $\sum_{m=1}^{\infty} m f_{M}(m)<\infty$; então, o resultado segue do Critério de Weierstrass (Rudin, 1976, p. 148).

A Equação (2.10) é equivalente a Equação (3) em Cooner et al. (2007), No entanto, a convergência uniforme da série em (2.10) não é dada ali e será usada para estabelecer outros resultados. O corolário seguinte estabelece um limitante superior para a função de densidade correspondente às unidades suscetíveis. Este resultado também não é dado em Cooner et al. (2007) nem encontrado na literatura. 
Corolário 2.5. $\forall t>0: f^{*}(t) \leq \frac{E(M)}{1-p_{0}} f_{a}(t)$.

Prova. Segue de (2.10), observando que $\left(\begin{array}{c}m-1 \\ k-1\end{array}\right) S_{a}(t)^{m-k} F_{a}(t)^{k-1} \leq 1$.

Observe-se que deste corolário não pode-se inferir que $f^{*}(t) \leq f_{a}(t)$ pois $\frac{E(M)}{1-p_{0}} \geq 1$ (como já foi estabelecido).

O corolário seguinte da a relação existente entre os valores iniciais e de longo prazo das funções de taxa de falha correspondentes às unidades suscetíveis e aos tempos de ativação.

Corolário 2.6. Sejam $h^{*}(\cdot)$ e $h_{a}(\cdot)$ as funções de taxa de falha das unidades suscetíveis e dos tempos de ativação, respectivamente. Se $E(M)<\infty$; então,

$$
\lim _{t \rightarrow 0^{+}} h^{*}(t)=\frac{E(M P(K=1 \mid M))}{1-p_{0}} \lim _{t \rightarrow 0^{+}} h_{a}(t)
$$

e

$$
\lim _{t \rightarrow \infty} h^{*}(t)=\lim _{t \rightarrow \infty} h_{a}(t)
$$

Prova. Desde que $E(M)<\infty$, a convergência uniforme da série em $t$ estabelecida no corolário 2.4 vale; logo de (2.10) segue que

$$
\begin{aligned}
\lim _{t \rightarrow 0^{+}} f^{*}(t) & =\frac{1}{1-p_{0}} \lim _{t \rightarrow 0^{+}} f_{a}(t) \sum_{m=1}^{\infty} \sum_{k=1}^{m} m\left(\begin{array}{c}
m-1 \\
k-1
\end{array}\right) \lim _{t \rightarrow 0^{+}}\left(S_{a}^{m-k}(t) F_{a}^{k-1}(t)\right) f(k) f_{K \mid M} f_{M}(m) \\
& =\frac{1}{1-p_{0}} \lim _{t \rightarrow 0^{+}} f_{a}(t) \sum_{m=1}^{\infty} m f_{K \mid M=m} f_{M}(m) \\
& =\frac{1}{1-p_{0}} \lim _{t \rightarrow 0^{+}} f_{a}(t) \sum_{m=1}^{\infty} m P(K=1 \mid M=m) f_{M}(m) \\
& =\frac{1}{1-p_{0}} \lim _{t \rightarrow 0^{+}} f_{a}(t) E(M P(K=1 \mid M)) \\
& =\frac{E(M P(K=1 \mid M))}{1-p_{0}} \lim _{t \rightarrow 0^{+}} h_{a}(t),
\end{aligned}
$$

onde a segunda igualdade segue de que $\lim _{t \rightarrow 0^{+}} S_{a}(t)=\lim _{t \rightarrow \infty} F_{a}(t)=1$ e a última igualdade segue do fato que $\lim _{t \rightarrow 0^{+}} h_{a}(t)=\lim _{t \rightarrow 0^{+}} f_{a}(t)$.

Para provar que $\lim _{t \rightarrow \infty} h^{*}(t)=\lim _{t \rightarrow \infty} h_{a}(t)$, observe-se que para toda função de taxa de falha $h$, com funções de densidade e de sobrevivência associadas $f(\cdot)$ e $S(\cdot)$, respectivamente,

$$
\lim _{t \rightarrow \infty} h(t)=\lim _{t \rightarrow \infty} \frac{f(t)}{S(t)}=-\lim _{t \rightarrow \infty} \frac{f^{\prime}(t)}{f(t)}
$$

pois, $\lim _{t \rightarrow \infty} f(t)=\lim _{t \rightarrow \infty} S(t)=0$. 
Por outra parte, também de (2.10) e da convergência uniforme da série em $t$ estabelecida no corolário 2.4, tem-se que $f^{*^{\prime}}(t)=f_{a}^{\prime}(t) f^{*}(t) / f_{a}(t)+g(t)$, onde $g(\cdot)$ é uma função com $\lim _{t \rightarrow \infty} g(t)=0$. De aqui e de (2.13), segue

$$
\lim _{t \rightarrow \infty} h^{*}(t)=-\lim _{t \rightarrow \infty} \frac{f^{*^{\prime}}(t)}{f^{*}(t)}=-\lim _{t \rightarrow \infty} \frac{f_{a}^{\prime}(t)}{f_{a}(t)}=\lim _{t \rightarrow \infty} h_{a}(t)
$$

Como foi visto, o número de fatores que precisam ativar-se, $K$, permite explicar o tempo de ocorrência do evento de interesse a partir de uma relação com o número de fatores latentes e seus correspondentes tempos de ativação; esta relação é conhecida na literatura como mecanismo de ativação, conforme denominado por Cooner et al. (2007). $\mathrm{Na}$ linguagem da teoria da confiabilidade, $T$ corresponderia a duração de um sistema tipo $k$ de $n$, com $k=K$ (o número mínimo de componentes que precisam funcionar para que o sistema funcione) e $n=M$ (o número de componentes do sistema) aleatórios.

A seguir são mostrados os mecanismos de ativação usuais na literatura e apresentados alguns resultados.

\subsection{Mecanismo da primeira ativação}

Neste caso $K=1$, ou seja, a primeira ativação de um dos fatores latentes origina a ocorrência do evento de interesse, assim

$$
T=\left\{\begin{array}{l}
\infty, \quad \text { se } M=0 \\
Z_{(1, M)}=\min \left\{Z_{1}, \ldots, Z_{M}\right\}, \quad \text { se } M \geq 1
\end{array}\right.
$$

Na linguagem da teoria da confiabilidade $T$ corresponderia à duração de um sistema em série com $M$ componentes, se $M \geq 1$.

De (2.3), as funções de sobrevivência e densidade das unidades suscetíveis, que denotaremos por $S_{F}^{*}(\cdot)$ e $f_{F}^{*}(\cdot)$, respectivamente, são dadas por

$$
S_{F}^{*}(t)=\frac{1}{1-p_{0}} \sum_{m=1}^{\infty} S_{a}^{m}(t) f_{M}(m),
$$

e

$$
f_{F}^{*}(t)=\frac{f_{a}(t)}{1-p_{0}} \sum_{m=1}^{\infty} m S_{a}^{m-1}(t) f_{M}(m),
$$


que também podem-se escrever como

$$
S_{F}^{*}(t)=\frac{g_{M}\left(S_{a}(t)\right)-p_{0}}{1-p_{0}}
$$

e

$$
f_{F}^{*}(t)=f_{a}(t) \frac{g_{M}^{\prime}\left(S_{a}(t)\right)}{1-p_{0}},
$$

em que $g_{M}(\cdot)$ é a função geradora de probabilidades de $M$.

A função de sobrevivência correspondente à população, $S_{p F}(t)=p_{0}+\left(1-p_{0}\right) S_{F}^{*}(t)$, é tal que

$$
S_{p F}(t)=\sum_{m=0}^{\infty} S_{a}^{m}(t) f_{M}(m)
$$

ou, equivalentemente,

$$
S_{p F}(t)=g_{M}\left(S_{a}(t)\right)=E\left(S_{a}^{M}(t)\right)
$$

O resultado de (2.21) é destacado em Tsodikov et al. (2003) e Rodrigues et al. (2009a).

\subsubsection{Algumas propriedades}

A seguir são apresentados resultados correspondentes a este esquema de ativação. Alguns destes resultados generalizam propriedades do sistema tipo série da teoria da confiabilidade, como será observado.

O Corolário seguinte estabelece a ordem estocástica entre as funções de sobrevivência dos tempos de ativação e a obtida como este mecanismo. Este resultado não é dado em Cooner et al. (2007).

Corolário 2.7. Independentemente da distribuição de $M$,

$$
\forall t>0: S_{F}^{*}(t) \leq S_{a}(t)
$$

Como consequência segue que

$$
\forall t>0: S_{p F}(t) \leq p_{o}+\left(1-p_{o}\right) S_{a}(t)
$$

Prova. Já que $\forall t>0, \forall m>0: S_{a}^{m}(t) \leq S_{a}(t)$, pois $0 \leq S_{a}(t) \leq 1$, segue de (2.16) que $S_{F}^{*}(t)=\frac{1}{1-p_{0}} \sum_{m=1}^{\infty} S_{a}^{m}(t) f_{M}(m) \leq \frac{1}{1-p_{0}} \sum_{m=1}^{\infty} S_{a}(t) f_{M}(m)=S_{a}(t)$. 
O corolário anterior generaliza o resultado muito conhecido da teoria da confiabilidade que o sistema em série possui uma confiabilidade menor ou igual do que as correspondentes aos componentes (se as durações destes for independentes e identicamente distribuídas).

Os corolários seguintes estabelecem propriedades da função de taxa de falha com este mecanismo de ativação.

Corolário 2.8. Sejam $h_{F}^{*}(\cdot)$ a função de taxa de falha das unidades suscetíveis, sob o esquema da primeira ativação, e $h_{a}(\cdot)$ a correspondente aos tempos de ativação. Se $E(M)<\infty$; então,

$$
\lim _{t \rightarrow 0^{+}} h_{F}^{*}(t)=\frac{E(M)}{1-p_{0}} \lim _{t \rightarrow 0^{+}} h_{a}(t) \geq \lim _{t \rightarrow 0^{+}} h_{a}(t)
$$

$e$

$$
\lim _{t \rightarrow \infty} h_{F}^{*}(t)=\lim _{t \rightarrow \infty} h_{a}(t)
$$

Prova. As igualdades seguem do corolário 2.6 e a desigualdade do fato que $E(M) \geq$ $1-p_{0}$.

Como mostra o corolário anterior estas propriedades são gerais, no entanto em muitos modelos propostos recentemente estes limites são calculados em cada caso particular.

Corolário 2.9. Para cada $m \in\{1,2, \ldots\}$, seja $f_{m}(\cdot)$ a densidade do mínimo de uma amostra aleatória de tamanho $m$ de uma variável aleatória com função de sobrevivência $S_{a}(\cdot)$, isto é, $f_{m}(t)=m S_{a}^{m-1}(t) f_{a}(t), \forall t>0$. Se a função de taxa de falha associada a esta densidade é decrescente $\forall m \in\{1,2, \ldots\}$; então, a função de taxa de falha das unidades suscetíveis $h_{F}^{*}(\cdot)$, sob o esquema da primeira ativação, também é decrescente.

Prova. Se $f_{F}^{*}(\cdot)$ denota a função de densidade das unidades suscetíveis, segue de (2.10) ou (2.16) que $\forall t>0$ :

$$
f_{F}^{*}(t)=\sum_{m=1}^{\infty} f_{m}(t) \frac{1}{1-p_{0}} f_{M}(m),
$$

$\operatorname{com} \sum_{m=1}^{\infty} \frac{1}{1-p_{0}} f_{M}(m)=1$, ou seja, $f_{F}^{*}(\cdot)$ é uma mistura de densidades cujas funções de taxa de falha são decrescentes. Assim, o resultado segue do Teorema 3.4 em Barlow et al. (1963, p. 381). 
O resultado deste corolário foi observado em Tahmasbi \& Rezaei (2008) e justifica por que muitos modelos propostos recentemente, em que se considera a distribuição exponencial o de Weibull, possuem funções de taxa de falha decrescente.

\subsubsection{Alguns casos particulares}

A seguir serão derivadas algumas funções de sobrevivência estendidas considerando em (2.20) ou (2.18) distribuições específicas para o número de fatores latentes $M$, com $P(M=0)>0$. As mesmas distribuições fixadas para $M$ são truncadas no zero para obter funções de sobrevivência usuais; neste caso será usado o fato que a função de sobrevivência obtida assim é a função de sobrevivência das unidades suscetíveis correspondente ao modelo de fatores latentes quando $M$ tem a distribuição não truncada, como estabelecido no corolário 2.1. A maioria das funções de sobrevivência que resultam foram apresentadas em diferentes trabalhos, mas sem usar a teoria dos modelos de fatores latentes.

a) Se $M$ segue uma distribuição de Bernoulli com $P(M=1)=1-\theta$ obtém-se o modelo de mistura. Neste caso $p_{0}=P(M=0)=\theta, S_{F}^{*}(t)=S_{a}(t)$ e

$$
S_{p F}(t)=\theta+(1-\theta) S_{a}(t) .
$$

b) Se $M$ segue uma distribuição de Poisson com parâmetro $\theta$ obtém-se o modelo de promoção. Neste caso $p_{0}=P(M=0)=e^{-\theta}, S_{F}^{*}(t)=\frac{1-e^{\theta S_{a}(t)}}{1-e^{\theta}} \mathrm{e}$, portanto,

$$
S_{p F}(t)=e^{-\theta\left(1-S_{a}(t)\right)} \text {. }
$$

Se $M$ segue uma distribuição de Poisson truncada no zero e com parâmetro $\theta$, isto é, $f_{M}(m)=\frac{e^{-\theta} \theta^{m}}{m !\left(1-e^{-\theta}\right)}, m=1,2, \ldots$; tem-se que $p_{0}=P(M=0)=0$; então, a função de sobrevivência resultante, $S_{p F}(\cdot)$, é dada por $S_{F}^{*}(\cdot)$ do caso anterior:

$$
S_{p F}(t)=\frac{1}{1-e^{-\theta}}\left(e^{-\theta\left(1-S_{a}(t)\right)}-e^{-\theta}\right)=\frac{1-e^{\theta S_{a}(t)}}{1-e^{\theta}} .
$$

Se os tempos de ativação seguem uma distribuição exponencial com parâmetro $\lambda$; obtém-se a família de distribuições, com taxa de risco monótona decrescente, proposta e estudada por Kus (2007).

c) Se $M$ segue uma distribuição geométrica com parâmetro $1-\theta$, tem-se $p_{0}=1-\theta$, $S_{F}^{*}(t)=\frac{(1-\theta) S_{a}(t)}{1-\theta S_{a}(t)} \mathrm{e}$, por tanto,

$$
S_{p F}(t)=\frac{1-\theta}{1-\theta S_{a}(t)}
$$


Se $M$ segue uma distribuição geométrica truncada no zero com parâmetro $1-\theta$, isto é, $f_{M}(m)=\theta^{m-1}(1-\theta), m=1,2, \ldots$; então, a função de sobrevivência resultante, $S_{p F}(\cdot)$, é dada por $S_{F}^{*}(\cdot)$ do caso anterior:

$$
S_{p F}(t)=\frac{(1-\theta) S_{a}(t)}{1-\theta S_{a}(t)} .
$$

Se os tempos de ativação seguem uma distribuição exponencial com parâmetro $\lambda$; obtém-se a família de distribuições, com taxa de risco monótona decrescente, proposta e estudada por Adamidis \& Loukas (1998).

d) Os modelos dos exemplos anteriores e mais outros podem ser generalizados usando uma distribuição mais geral para $M$, por exemplo, a de série de potências:

$$
f_{M}(m)=P(M=m)=\frac{a_{m} \theta^{m}}{C(\theta)}, m \in\{0,1,2, \ldots\} ; \theta \in \Theta,
$$

em que $a_{0}, a_{1}, \ldots$ é uma seqüência de números reais não negativos, com pelo menos um destes positivo, $\Theta=(0, r)$, com $r$ um número positivo não maior do que o raio de convergência da série $\sum_{m=0}^{\infty} a_{m} \theta^{m}$, e $C$ é a função série de potências definida por $C(\theta)=\sum_{m=0}^{\infty} a_{m} \theta^{m}, \forall \theta \in(0, r)$. Note, em particular, que $C$ é positiva e infinitamente diferenciável. As distribuições Poisson, geométrica, logarítmica, entre outras, são casos particulares desta distribuição.

A função geradora de probabilidades desta distribuição é

$$
g(s)=\frac{C(\theta s)}{C(\theta)}, \text { se } s r \in \Theta
$$

A fração de cura neste modelo é $p_{0}=P(M=0)=\frac{a_{0}}{C(\theta)}$ e a função de sobrevivência, obtida de (2.20) ou de (2.18), é dada por

$$
S_{p F}(t)=\frac{C\left(\theta S_{a}(t)\right)}{C(\theta)}, \forall t>0 ; \forall \theta \in \Theta .
$$

Observe-se que $\theta S_{a}(t) \in(0, r)=\Theta$, considerando $0<S_{a}(t) \leq 1$.

Se $M$ segue uma distribuição de série de potências truncada no zero; então, a função de sobrevivência resultante e dada por

$$
S_{p F}(t)=\frac{C\left(\theta S_{a}(t)\right)-a_{0}}{C(\theta)-a_{0}}, \forall t>0 ; \forall \theta \in \Theta .
$$

Se os tempos de ativação seguem uma distribuição exponencial, com parâmetro $\lambda$, obtém-se a família de distribuições, com taxa de risco monótona decrescente, proposta e estudada por Chahkandi \& Ganjali (2009):

$$
S_{p F}(t)=\frac{C\left(\theta e^{-\lambda t}\right)-a_{0}}{C(\theta)-a_{0}}, \forall t>0 ; \forall \theta \in \Theta, \lambda>0 .
$$


Morais \& Barreto-Souza (2011) consideraram a distribuição de Weibull e estudaram as propriedades da distribuição associada à função de sobrevivência obtida:

$$
S_{p F}(t)=\frac{C\left(\theta e^{-(\lambda t)^{\alpha}}\right)-a_{0}}{C(\theta)-a_{0}}, \forall t>0 ; \forall \theta \in \Theta, \lambda>0, \alpha>0 .
$$

e) Se $M$ tem distribuição COM-Poisson, isto é, sua função de distribuição de probabilidades é

$$
f_{M}(m)=\frac{\theta^{m}}{(m !)^{\phi} H(\theta, \phi)}, m=0,1, \ldots
$$

em que $\phi>0, \theta>0$ e $H(\theta, \phi)=\sum_{j=0}^{\infty} \frac{\theta^{j}}{(j !)^{\phi}}$ (note que esta série é finita).

A função geradora de probabilidades desta distribuição é

$$
g(s)=\frac{H(\theta s, \phi)}{H(\theta \phi)}, \forall s>0
$$

A fração de cura é $p_{0}=P(M=0)=\frac{1}{H(\theta, \phi)}$. Assim, de (2.20) ou de (2.18), obtém-se o modelo com fração de cura COM-Poisson proposto e estudado por Rodrigues et al. (2009b):

$$
S_{p F}(t)=\frac{H\left(\theta S_{a}(t), \phi\right)}{H(\theta, \phi)} .
$$

O modelo do caso b, ou seja, com a distribuição de Poisson, corresponde ao caso particular de $\phi=1$. Se $\phi<1$ tem-se uma distribuição que modela a sobredispersão e se $\phi>1$ modela-se a sub-dispersão.

Se $M$ tem distribuição COM-Poisson truncada no zero, a função de sobrevivência correspondente e dada por

$$
S_{p 1 F}(t)=\frac{H\left(\theta S_{a}(t), \phi\right)-\frac{1}{H(\theta, \phi)}}{1-H(\theta, \phi)} .
$$

Aparentemente não existem referências na literatura sobre esta função de sobrevivência.

f) Uma família de distribuições ainda mais geral que as de série de potências e COM-Poisson é a distribuição Poisson ponderada, esta foi usada no trabalho de Rodrigues et al. (2010). Neste caso

$$
f(m)=P(M=m)=\frac{\mathrm{w}(m ; \phi) \frac{e^{-\theta} \theta^{m}}{m !}}{D(\theta, \phi)}, m=0,1,2, \ldots ; \theta \in \Theta
$$


sendo $\mathrm{w}(\cdot ; \phi)$ uma função não negativa (chamada função peso), $\phi>0$ (o parâmetro da função peso), $\Theta=(0, r)$, com $r>0$ um número tal que a série $\sum_{j=0}^{\infty} \frac{\mathrm{w}(j ; \phi) \theta^{j}}{j !}$ convirja, $\forall \phi>0, \forall \theta \in \Theta$, e $D(\theta, \phi)=\sum_{j=0}^{\infty} \mathrm{w}(j ; \phi) \frac{e^{-\theta} \theta^{j}}{j !}, \forall \phi>0, \forall \theta \in \Theta$.

As distribuições Poisson ponderadas constituem uma família geral que inclui, entre outras, a distribuição binomial negativa (obtém-se quando $\mathrm{w}(m ; \phi)=$ $\Gamma(m+\phi)$, com $\phi>0$ e $0<\theta<1$ ), a distribuição COM-Poisson (que resulta quando $\left.\mathrm{w}(m ; \phi)=(m !)^{1-\phi}\right)$ e distribuição de série de potências (obtémse quando $\left.\mathrm{w}(m, \phi)=\mathrm{w}(m)=m ! a_{m}\right)$. Outras propriedades são obtidas em Balakrishnan (2008).

Se $M$ tem uma distribuição Poisson ponderada, com função de probabilidades dada em (2.38), então sua função geradora de probabilidades é dada por

$$
g(s)=\sum_{m=0}^{\infty} s^{m} \frac{\mathrm{w}(m ; \phi) \frac{e^{-\theta} \theta^{m}}{m !}}{D(\theta, \phi)}=\frac{e^{-\theta(1-s)} D(s \theta, \phi)}{D(\theta, \phi)},
$$

para $s$ tal que $s \theta<r$.

Assim, para este modelo $p_{0}=P(M=0)=\frac{e^{-\theta} \mathrm{w}(0, \phi)}{D(\theta, \phi)}$ e a função de sobrevivência, obtida de (2.18) e (2.39), é

$$
S_{p F}(t)=\frac{e^{-\theta\left(1-S_{a}(t)\right)} D\left(S_{a}(t) \theta, \phi\right)}{D(\theta, \phi)} .
$$

Se $M$ tem distribuição ponderada truncada no zero, a função de sobrevivência que se obtém é

$$
S_{p F}(t)=\frac{e^{-\theta\left(1-S_{a}(t)\right)} D\left(S_{a}(t) \theta, \phi\right)-e^{-\theta} \mathrm{w}(0, \phi)}{D(\theta, \phi)-e^{-\theta} \mathrm{w}(0, \phi)} .
$$

Aparentemente não existem referências na literatura sobre esta função de sobrevivência.

\subsection{Mecanismo da última ativação}

Neste caso $K=M$, se $M>0$, ou seja, a última ativação dos fatores latentes origina a ocorrência do evento de interesse, assim

$$
T=\left\{\begin{array}{l}
\infty, \quad \text { se } M=0, \\
Z_{(M, M)}=\max \left\{Z_{1}, \ldots, Z_{M}\right\}, \quad \text { se } M \geq 1 .
\end{array}\right.
$$


Na linguagem da teoria da confiabilidade $T$ corresponderia à duração de um sistema em paralelo com $M$ componentes, se $M \geq 1$.

De (2.3), a função de sobrevivência dos indivíduos suscetíveis é dada por

$$
S_{L}^{*}(t)=1-\frac{1}{1-p_{0}} \sum_{m=1}^{\infty} F_{a}^{m}(t) f_{M}(m),
$$

ou, equivalentemente,

$$
S_{L}^{*}(t)=\frac{1-g_{M}\left(F_{a}(t)\right)}{1-p_{0}},
$$

em que $g_{M}$ é a função geradora de probabilidades de $M$. Assim, a função de sobrevivência correspondente à população, $S_{p L}(t)=p_{0}+\left(1-p_{0}\right) S_{L}^{*}(t)$, resulta

$$
S_{p L}(t)=1+p_{0}-\sum_{m=0}^{\infty} F_{a}^{m}(t) f_{M}(m)
$$

ou, equivalentemente,

$$
S_{p L}(t)=1+p_{0}-g_{M}\left(F_{a}(t)\right)
$$

\subsubsection{Algumas propriedades}

A seguir são apresentados resultados correspondentes a este esquema de ativação, não deduzidos em Cooner et al. (2007). O Corolário 2.10 estabelece a ordem estocástica entre as funções de sobrevivência obtida com este mecanismo e a correspondente aos tempos de ativação.

Corolário 2.10. Independentemente da distribuição de $M$,

$$
\forall t>0: S_{L}^{*}(t) \geq S_{a}(t)
$$

Como consequência segue que

$$
\forall t>0: S_{p F}(t) \leq p_{o}+\left(1-p_{o}\right) S_{a}(t)
$$

Prova. Já que $\forall t>0, \forall m>0: F_{a}^{m}(t) \leq F_{a}(t)$, pois $0 \leq F_{a}(t) \leq 1$, segue de (2.43) que $S_{L}^{*}(t)=1-\frac{1}{1-p_{0}} \sum_{m=1}^{\infty} F_{a}^{m}(t) f_{M}(m) \geq 1-\frac{1}{1-p_{0}} \sum_{m=1}^{\infty} F_{a}(t) f_{M}(m)=1-F_{a}(t)$.

O corolário anterior generaliza o resultado muito conhecido da teoria da confiabilidade que o sistema em paralelo possui uma confiabilidade maior ou igual 
do que as correspondentes aos componentes (se as durações destes for independentes e identicamente distribuídas).

Como conseqüência dos corolários 2.7 e 2.10 segue a ordem estocástica entre as funções de sobrevivência dos mecanismos da primeira e última ativação.

Corolário 2.11. Considere-se os mecanismos da primeira e da última ativação, ambos com a mesma função de sobrevivência, $S_{a}(\cdot)$, dos tempos de ativação dos fatores de risco; então, para quaisquer que sejam as distribuições do número de fatores de risco latentes usadas tem-se que

$$
\forall t>0: S_{F}^{*}(t) \leq S_{a}(t) \leq S_{L}^{*}(t)
$$

Um resultado similar é dado para o modelo proposto em Kim et al. (2011) especificado em 2.6.

Os corolários seguintes estabelecem propriedades da função de taxa de falha das unidades suscetíveis para este mecanismo de ativação.

Corolário 2.12. Sejam $h_{L}^{*}(\cdot)$ a função de taxa de falha das unidades suscetiveis, sob o esquema da primeira ativação, e $h_{a}(\cdot)$ a correspondente aos tempos de ativação. Se $E(M)<\infty$; então,

$$
\begin{aligned}
& \lim _{t \rightarrow 0^{+}} h_{L}^{*}(t)=\frac{P(M=1)}{1-p_{0}} \lim _{t \rightarrow 0^{+}} h_{a}(t) \leq \lim _{t \rightarrow 0^{+}} h_{a}(t), \\
& \lim _{t \rightarrow \infty} h_{L}^{*}(t)=\lim _{t \rightarrow \infty} h_{a}(t) .
\end{aligned}
$$

Prova. Do corolário 2.6 resultam as igualdades. Por outra parte, de $P(M=1) \leq 1-p_{0}$ segue a desigualdade.

Como mostra o corolário anterior estas propriedades são gerais, no entanto em muitos modelos propostos recentemente são verificadas em cada caso particular.

Os resultados seguintes tratam da monotonia da taxa de risco com este mecanismo de ativação.

Corolário 2.13. A função de taxa de falha, $h_{L}^{*}(\cdot)$, é decrescente para t suficientemente pequeno se e somente se

$$
\left[f_{a}^{\prime}\left(0^{+}\right) P(M=1)+2 f_{a}^{2}\left(0^{+}\right) P(M=2)\right]\left(1-p_{0}\right)+f_{a}^{2}\left(0^{+}\right) P^{2}(M=1)<0,
$$

$\operatorname{com} f_{a}\left(0^{+}\right)=\lim _{t \rightarrow 0^{+}} f_{a}(t)$ e $f_{a}^{\prime}\left(0^{+}\right)=\lim _{t \rightarrow 0^{+}} f_{a}^{\prime}(t)$. 
Prova. De (2.44) segue que

$$
h_{L}^{*}(t)=\frac{f_{a}(t) g_{M}^{\prime}\left(F_{a}(t)\right)}{1-g_{M}\left(F_{a}(t)\right)} .
$$

Assim, a derivada de $h_{L}^{*}$ é dada por

$$
h_{L}^{* \prime}(t)=\frac{\left[f_{a}^{\prime}(t) g_{M}^{\prime}\left(F_{a}(t)\right)+f_{a}^{2}(t) g_{M}^{\prime \prime}\left(F_{a}(t)\right)\right]\left[1-g_{M}\left(F_{a}(t)\right)\right]+f_{a}^{2}(t) g_{M}^{\prime 2}\left(F_{a}(t)\right)}{\left[1-g_{M}\left(F_{a}(t)\right)\right]^{2}},
$$

daqui segue o resultado, pois

$$
\lim _{t \rightarrow 0^{+}} h_{L}^{* \prime}(t)=\frac{\left[f_{a}^{\prime}\left(0^{+}\right) P(M=1)+2 f_{a}^{2}\left(0^{+}\right) P(M=2)\right]\left(1-p_{0}\right)+f_{a}^{2}\left(0^{+}\right) P^{2}(M=1)}{\left(1-p_{0}\right)^{2}} .
$$

No lema seguinte é considerado que os tempos de ativação dos fatores de risco tenham uma distribuição exponencial. Depois o resultado é generalizado para uma distribuição cuja função de taxa de risco seja crescente.

Lema 2.1. Se $E\left(M^{2}\right)$ é finito e os tempos de ativação seguem uma distribuição exponencial; então, A função de taxa de falha, $h_{L}^{*}(\cdot)$, é crescente para t suficientemente grande.

Prova. De (2.49) segue que a derivada de $h_{L}^{*}$ é dada por

$$
h_{L}^{* \prime}(t)=\frac{\lambda^{2} e^{-\lambda t} w(t)}{\left[1-g_{M}\left(F_{a}(t)\right)\right]^{2}},
$$

onde $\lambda>0$ é o parâmetro da distribuição exponencial e $w(t)$ está definida, para $t>0$, como

$$
w(t)=\left[-g_{M}^{\prime}\left(F_{a}(t)\right)+e^{-\lambda t} g_{M}^{\prime \prime}\left(F_{a}(t)\right)\right]\left[1-g_{M}\left(F_{a}(t)\right)\right]+e^{-\lambda t} g_{M}^{\prime}{ }^{2}\left(F_{a}(t)\right) .
$$

Observe-se que $\lim _{t \rightarrow \infty} w(t)=0$; logo, o resultado segue demonstrando que $w^{\prime}(t)<0$, para $t$ suficientemente grande. Mas $w^{\prime}(t)=\lambda e^{-\lambda t} w_{1}(t)$, onde

$$
\begin{aligned}
w_{1}(t)= & {\left[-2 g_{M}^{\prime \prime}\left(F_{a}(t)\right)+e^{-\lambda t} g_{M}^{\prime \prime \prime}\left(F_{a}(t)\right)\right]\left[1-g_{M}\left(F_{a}(t)\right)\right] } \\
& +e^{-\lambda t} g_{M}^{\prime}\left(F_{a}(t)\right) g_{M}^{\prime \prime}\left(F_{a}(t)\right)
\end{aligned}
$$

e $\lim _{t \rightarrow \infty} w_{1}(t)=0$. Assim, é suficiente demonstrar que $w_{1}^{\prime}(t)$ é positiva para $t$ suficientemente grande. Agora, observando que $w_{1}^{\prime}(t)=\lambda e^{-\lambda t} w_{2}(t)$, em que

$$
\begin{aligned}
w_{2}(t)= & {\left[-3 g_{M}^{\prime \prime \prime}\left(F_{a}(t)\right)+e^{-\lambda t} g_{M}^{(i v)}\left(F_{a}(t)\right)\right]\left[1-g_{M}\left(F_{a}(t)\right)\right] } \\
& +g_{M}^{\prime}\left(F_{a}(t)\right) g_{M}^{\prime \prime}\left(F_{a}(t)\right)+e^{-\lambda t} g_{M}^{\prime \prime 2}\left(F_{a}(t)\right)
\end{aligned}
$$


e $\lim _{t \rightarrow \infty} w_{2}(t)=E(M) E(M(M-1))>0$; então, $w_{2}(t)$ e $w_{1}^{\prime}(t)$ são positivos para $t$ suficientemente grande.

Corolário 2.14. Se $E\left(M^{2}\right)$ é finito e os tempos de ativação têm uma função de taxa de falha, $h_{a}(\cdot)$, crescente; então, a função de taxa de falha das unidades suscetíveis, $h_{L}^{*}(\cdot)$, é crescente para $t$ suficientemente grande.

Prova. Como $h_{a}(\cdot)$ es crescente, então, $F_{a}(t)=1-e^{-H(t)}, \forall t>0$, com $H(\cdot)$ uma função positiva crescente e convexa (vide Barlow et al. (1963, p. 378)). Assim, de (2.44) segue que

$$
h_{L}^{*}(t)=\frac{H^{\prime}(t) e^{-H(t)} g_{M}^{\prime}\left(1-e^{-H(t)}\right)}{1-g_{M}\left(1-e^{-H(t)}\right)} .
$$

Agora, seja $h_{L e}^{*}(\cdot)$ a função de taxa de falha das unidades suscetíveis, quando os tempos de ativação dos fatores de risco latentes têm uma distribuição exponencial. Segue, também de (2.44), que

$$
h_{L e}^{*}(t)=\frac{\lambda e^{-\lambda t} g_{M}^{\prime}\left(1-e^{-\lambda t}\right)}{1-g_{M}\left(1-e^{-\lambda t}\right)} .
$$

Assim, das últimas duas equações tem-se que

$$
h_{L e}^{*}\left(\lambda^{-1} H(t)\right)=\frac{\lambda e^{-H(t)} g_{M}^{\prime}\left(1-e^{-H(t)}\right)}{1-g_{M}\left(1-e^{-H(t)}\right)}=\frac{\lambda}{H^{\prime}(t)} h_{L}^{*}(t),
$$

ou seja,

$$
h_{G}^{*}(t)=\frac{H^{\prime}(t)}{\lambda} h_{L e}^{*}\left(\lambda^{-1} H(t)\right) .
$$

Logo, como $H^{\prime}$ é uma função crescente (pois $H$ é convexa), o resultado segue do lema 2.1 e da última equação.

\subsubsection{Alguns casos particulares}

Com as distribuições específicas para o número de fatores de risco latentes, $M$, consideradas em 2.2.2, serão deduzidas funções de sobrevivência utilizando (2.45) ou (2.46) do modelo de fatores latentes. A maioria das funções de sobrevivência que resultam foram apresentadas em diferentes trabalhos, mas sem usar os resultados da teoria dos modelos de fatores latentes.

a) Se $M$ segue uma distribuição de Bernoulli com $P(M=1)=1-\theta$, o resultado é o mesmo da primeira ativação, ou seja, o modelo de mistura de Boag (1949) e Berkson \& Gage (1952):

$$
S_{p L}(t)=\theta+(1-\theta) S_{a}(t) .
$$


b) Se $M$ segue uma distribuição de Poisson com parâmetro $\theta$, o resultado é diferente do obtido com a primeira ativação. Neste caso $p_{0}=P(M=0)=e^{-\theta}, S_{L}^{*}(t)=$ $\frac{1}{1-e^{-\theta}}\left[1-e^{-\theta S_{a}(t)}\right] \mathrm{e}$

$$
S_{p L}(t)=1+e^{-\theta}\left[1-e^{\theta F_{a}(t)}\right] .
$$

Se $M$ segue uma distribuição de Poisson truncada no zero e com parâmetro $\theta$; então, a função de sobrevivência resultante, $S_{p L}$, é dada por $S_{L}^{*}$ do caso anterior

$$
S_{p L}(t)=\frac{1}{1-e^{-\theta}}\left[1-e^{-\theta S_{a}(t)}\right] .
$$

Se os tempos de ativação seguem uma distribuição exponencial com parâmetro $\lambda$; obtém-se a família de distribuições (com taxa de risco monótona crescente) proposta e estudada por Cancho et al. (2011a).

c) Se $M$ segue uma distribuição geométrica com parâmetro $1-\theta$, tem-se $p_{0}=1-\theta$, $S_{L}^{*}(t)=\frac{1-F_{a}(t)}{1-\theta F_{a}(t)} \mathrm{e}$

$$
S_{p L}(t)=1-\theta+\theta \frac{1-F_{a}(t)}{1-\theta F_{a}(t)} .
$$

Se $M$ segue uma distribuição geométrica truncada no zero com parâmetro $1-\theta$, a função de sobrevivência resultante é dada por $S_{L}^{*}$ do caso anterior

$$
S_{p L}=\frac{1-F_{a}(t)}{1-\theta F_{a}(t)} .
$$

Se os tempos de ativação seguem uma distribuição exponencial com parâmetro $\lambda$; obtém-se a família de distribuições (com taxa de risco monótona decrescente) proposta e estudada por Adamidis et al. (2005).

d) Se $M$ tem uma distribuição de série de potências, como definido em (2.28) no caso da primeira ativação, tem-se que $p_{0}=P(M=0)=\frac{a_{0}}{C(\theta)}$. A função de sobrevivência com a última ativação, obtida de (2.45), é

$$
S_{p L}(t)=1+\frac{a_{0}}{C(\theta)}-\frac{C\left(\theta F_{a}(t)\right)}{C(\theta)}, \forall t>0 ; \forall \theta \in \Theta .
$$

Observe-se que $\theta F_{a}(t) \in \Theta$, desde que $0<F_{a}(t) \leq 1$.

No Capítulo 4 é tratado este modelo considerando que os tempos de ativação seguem uma distribuição exponencial generalizada.

Se é usada a distribuição truncada no zero a função de sobrevivência resultante pode ser obtida do corolário 2.1:

$$
S_{p L}(t)=\frac{C(\theta)-C\left(\theta F_{a}(t)\right)}{C(\theta)-a_{0}}, \forall t>0 ; \forall \theta \in \Theta .
$$

No Capítulo 3 é feito um estudo desta família. 
e) Se $M$ tem distribuição COM-Poisson, temos que $p_{0}=P(M=0)=\frac{1}{H(\theta, \phi)}$ (como foi visto no quarto caso particular da primeira ativação) e a função de sobrevivência da população (obtida de (2.45)) é

$$
S_{p L}(t)=1+\frac{1}{H(\theta, \phi)}-\frac{H\left(\theta F_{a}(t), \phi\right)}{H(\theta, \phi)} .
$$

Se é usada a distribuição truncada no zero a função de sobrevivência resultante, $S_{p L}$ pode ser obtida do corolário 2.1

$$
S_{p 1 L}(t)=\frac{H(\theta, \phi)-H\left(\theta F_{a}(t), \phi\right)}{H(\theta, \phi)-1} .
$$

Não existem muitas referências na literatura a respeito destes modelos, mas sim de casos particulares como a distribuição de Poisson. Entretanto são importantes pois consideram a sobre-dispersão $(\phi>1)$ e a sub-dispersão $(\phi>1)$ que poderia ter o número de fatores latentes, $M$.

f) Se $M$ tem uma distribuição Poisson ponderada tem-se que $p_{0}=P(M=0)=$ $\frac{e^{-\theta} \mathrm{w}(0, \phi)}{D(\theta ; \phi)}$ (como foi visto no caso da primeira ativação). A função de sobrevivência, obtida de (2.45) e (2.39), é

$$
S_{p L}(t)=\frac{e^{-\theta F_{a}(t)} D\left(\theta S_{a}(t) ; \phi\right)}{D(\theta ; \phi)} .
$$

Se é usada a distribuição truncada no zero a função de sobrevivência resultante, $S_{p L}$, pode ser obtida do corolário 2.1

$$
S_{p L}(t)=\frac{e^{-\theta F_{a}(t)} D\left(\theta S_{a}(t), \phi\right)-e^{-\theta} \mathrm{w}(0, \phi)}{D(\theta, \phi)-e^{-\theta} \mathrm{w}(0, \phi)}
$$

Aparentemente não existem maiores referências na literatura a respeito destas duas famílias flexíveis de modelos.

\subsection{Mecanismo aleatório com distribuição uniforme}

Neste caso, a distribuição condicional do número de fatores latentes que devem-se ativar para que ocorra o evento de interesse, dado que o número de fatores é igual a $m$, tem distribuição uniforme, isto é, a $k$-ésima ativação produz a ocorrência do evento de interesse, com probabilidade $1 / m$. Assim, $\underset{K \mid M=m}{f(k)=\frac{1}{m}}, k=1, \ldots, m ; m \in\{1,2, \ldots\}$. 
Teorema 2.2. No mecanismo aleatório com distribuição uniforme, a função de sobrevivência do modelo de fatores latentes corresponde a um modelo de mistura em que a função de sobrevivência das unidades suscetiveis é a correspondente aos tempos de ativação, $S_{a}(\cdot)$, e a fração de cura é dada por $p_{0}=P(M=0)$; isto é,

$$
S_{p}(\cdot)=p_{0}+\left(1-p_{0}\right) S_{a}(\cdot)
$$

Observe que neste caso a distribuição de $M$ somente influi na determinação da fração de cura $p_{0}$.

Prova. O resultado segue de (2.3) e (2.9):

$$
\begin{aligned}
S^{*}(t) & =\frac{1}{1-p_{0}} \sum_{m=1}^{\infty} \sum_{k=1}^{m} \sum_{j=m-k+1}^{m}\left(\begin{array}{c}
m \\
j
\end{array}\right) S_{a}^{j}(t)\left[1-S_{a}(t)\right]^{m-j} f_{K \mid M=m}(k) f_{M}(m) \\
& =\frac{1}{1-p_{0}} \sum_{m=1}^{\infty} \sum_{k=1}^{m} \sum_{j=m-k+1}^{m}\left(\begin{array}{c}
m \\
j
\end{array}\right) S_{a}^{j}(t)\left[1-S_{a}(t)\right]^{m-j} \frac{1}{m} f_{M}(m) \\
& =\frac{1}{1-p_{0}} \sum_{m=1}^{\infty} m S_{a}(t) \frac{1}{m} f_{M}(m) \\
& =S_{a}(t),
\end{aligned}
$$

portanto $S_{p}(t)=p_{0}+\left(1-p_{0}\right) S_{a}(t)$. 


\section{Capítulo 3}

\section{Uma família de distribuições exponencial-série de potências}

\subsection{Introdução}

Muitas das distribuições conhecidas na teoria de sobrevivência tem surgido mediante modificações ou misturas de outras já existentes. Recentemente Chahkandi \& Ganjali (2009) propuseram a distribuição exponencial-série de potência (ESP), baseada na composição da distribuição exponencial com a de série de potências truncada no zero, que generaliza muitas propostas deste tipo para modelar uma taxa de risco decrescente. A distribuição anterior pode ser deduzida com a teoria dos modelos de fatores latentes com o esquema da primeira ativação, como foi feito no quarto exemplo da Seção 2.2.

Neste Capítulo é usada a teoria dos modelos de fatores latentes com o esquema da última ativação para deduzir uma família de distribuições. Também é assumido que o número de fatores de risco latentes segue uma distribuição de série de potências truncada no zero e os tempos de ativação destes seguem uma distribuição exponencial. A distribuição assim obtida, para o tempo de ocorrência do evento de interesse, será chamada de exponencial-série de potências complementar (ESPC) e generaliza os trabalhos de Adamidis et al. (2005), com a distribuição geométrica, e de Cancho et al. (2011a), com a distribuição de Poisson. A maioria dos resultados apresentados aqui produziram o artigo de Flores Delgado et al. (2013). Alguns dos resultados do artigo anterior foram, neste estudo, melhorados a partir dos novos resultados que foram apresentados no Capítulo 2. Como aplicação foi ajustado o modelo proposto a um conjunto de dados reais correspondentes a tempos de vida, da área de sobrevivência, que parecem apresentar uma taxa de risco crescente. Estimativas de máxima de 
verossimilhaça dos parâmetros de alguns membros da família ESPC foram obtidos. Após determinar o melhor modelo, foram obtidos os erros padrões de estimação e intervalos de confiança mediante simulação bootstrap (Efron \& Tibshirani, 1986). Não foram usadas as distribuições assintóticas dos estimadores de máxima verossimilhança, pois o tamanho da amostra é relativamente pequeno. Também foi realizado um estudo de simulação para amostras de tamanho pequeno.

\subsection{Uma dedução do modelo}

Além das suposições consideradas no modelo de fatores latentes com o esquema da última ativação, considere-se que o número de fatores latentes, $M$, segue uma distribuição de série de potências truncada no zero. Assim, segue de (2.28) que a função de distribuição de probabilidades de $M$ é dada por

$$
f_{M}(m)=P(M=m)=\frac{a_{m} \theta^{m}}{C(\theta)-a_{0}}, m \in\{1,2, \ldots\} ; \theta \in \Theta
$$

em que a seqüência de números $a_{0}, a_{1}, \ldots$, o conjunto $\Theta$ e a função série de potências $C(\theta)=\sum_{m=0}^{\infty} a_{m} \theta^{m}, \forall \theta \in \Theta$, foram descritas em (2.28). As distribuições truncadas Poisson, binomial, geométrica, binomial negativa, logarítmica, entre outras, são casos particulares da distribuição de séries de potências truncada. Na tabela seguinte são mostrados alguns casos particulares.

Tabela 3.1: Alguns casos particulares da distribuição de série de potências.

\begin{tabular}{|c|c|c|c|c|c|}
\hline Distribuição & $f(m)$ & & $\overline{a_{m}}$ & $C(\theta)$ & $\Theta$ \\
\hline Poisson & $\frac{\frac{\theta^{m}}{m !}}{e^{\theta}-1}, m=1,2, \ldots$ & & $\frac{1}{m !}$ & $e^{\theta}-1$ & $(0, \infty)$ \\
\hline Geométrica & $\frac{\theta^{m}}{(1-\theta)^{-1}-1}, m=1,2,$. & & 1 & $(1-\theta)^{-1}-1$ & $(0,1)$ \\
\hline Logarítmica & $\frac{\frac{\theta^{m}}{m}}{-\log (1-\theta)}, m=1,2, \ldots$ & & $\frac{1}{m}$ & $-\log (1-\theta)$ & $(0,1)$ \\
\hline Binomial & $\frac{\left(\begin{array}{c}n_{0} \\
m\end{array}\right) \theta^{m}}{(1+\theta)^{n_{0}}-1}, m=1,2, \ldots, n_{0}$ & $\begin{array}{c}\left(\begin{array}{c}n_{0} \\
m\end{array}\right) \\
0\end{array}$ & $\begin{array}{l}m \leq n_{0} \\
m>n_{0}\end{array}$ & $(1+\theta)^{n_{0}}-1$ & $(0,1)$ \\
\hline
\end{tabular}

Note-se que em cada caso a correspondente série de potências converge para $\theta \in \Theta$. Na distribuição binomial $n_{0} \in \mathbb{N}^{+}$é fixo (corresponde ao número de sucessos). 
Assuma-se também que os tempos de ativação seguem uma distribuição exponencial com parâmetro $\lambda$. Assim, $S_{a}(t)=e^{-\lambda t}, t>0$.

Com estas suposições, a função de sobrevivência de $T$, que será denotada por $S$, é dada por

$$
S(t)=\frac{C(\theta)-C\left(\theta\left(1-e^{-\lambda t}\right)\right)}{C(\theta)-a_{0}}, t>0 ; \theta \in \Theta, \lambda>0,
$$

como estabelecido em (2.56) no quarto exemplo da Seção 2.3.2.

A função de densidade de $T$ está dada por

$$
f(t)=\frac{\theta \lambda e^{-\lambda t} C^{\prime}\left(\theta\left(1-e^{-\lambda t}\right)\right)}{C(\theta)-a_{0}}, t>0 ; \theta \in \Theta, \lambda>0,
$$

com $C^{\prime}\left(\theta\left(1-e^{-\lambda t}\right)\right)$ a derivada de $C(\cdot)$ avaliada em $\theta\left(1-e^{-\lambda t}\right)$.

Esta densidade corresponde a distribuição Exponencial-Série de Potências complementar, com parâmetros $\theta$ e $\lambda$, e será denotada por $\operatorname{ESPC}(\theta, \lambda)$.

Note-se que

$$
f(t)=\sum_{m=1}^{\infty} \frac{a_{m} \theta^{m}}{C(\theta)} m \lambda e^{-\lambda t}\left(1-e^{-\lambda t}\right)^{m-1},
$$

isto é, $f$ é uma mistura das densidades $f \underset{m}{f} t ; \lambda)=m \lambda e^{-\lambda t}\left(1-e^{-\lambda t}\right)^{m-1}, t>0 ; \lambda>0$.

A função de distribuição cumulativa da família $\operatorname{ESPC}(\theta, \lambda)$ é dada por

$$
F(t)=\frac{C\left(\theta\left(1-e^{-\lambda t}\right)\right)-a_{0}}{C(\theta)-a_{0}}, t>0 ; \theta \in \Theta, \lambda>0,
$$

De (3.5), a função quantílica da distribuição $E S P C$ é dada por

$$
Q(u)=-\frac{1}{\lambda} \ln \left(1-\frac{1}{\theta} C^{-1}\left(u C(\theta)-u a_{0}\right)\right), 0<u<1 ; \theta \in \Theta, \lambda>0 .
$$

Observe-se que $C^{\prime}(\theta)=\sum_{m=1}^{\infty} m a_{m} \theta^{m-1}>0, \forall \theta \in \Theta$; então, existe $C^{-1}(\theta)$.

A função de taxa de falha da família $\operatorname{ESPC}(\theta, \lambda)$ é dada, segundo (3.3) e (3.6), por

$$
h(t)=\frac{\theta \lambda e^{-\lambda t} C^{\prime}\left(\theta\left(1-e^{-\lambda t}\right)\right)}{C(\theta)-C\left(\theta\left(1-e^{-\lambda t}\right)\right)}, t>0 ; \theta \in \Theta, \lambda>0 .
$$

\subsection{Algumas propriedades do modelo}

A seguir são dadas algumas propriedades do modelo $E S P C$.

A proposição seguinte mostra que a distribuição exponencial é obtida como um limite da distribuição $E S P C$, quando $a_{1}>0$. 
Proposição 3.1. Se $a_{1}>0$, a distribuição exponencial com parâmetro $\lambda$ é o limite da distribuição ESPC quando $\theta \rightarrow 0^{+}$. Em geral, $\lim _{\theta \rightarrow 0^{+}} F(t ; \theta, \lambda)=\left(1-e^{-\lambda t}\right)^{k_{0}}$, em que $k_{0}=\min \left\{m \in \mathbb{N}^{+}: a_{m}>0\right\}$.

Prova. De (3.5) e usando a regra de L'Hospital $k_{0}$ vezes, segue que $\lim _{\theta \rightarrow 0^{+}} F(t ; \theta, \lambda)=\lim _{\theta \rightarrow 0^{+}} \frac{\left(1-e^{-\lambda t}\right)^{k_{0}} C^{\left(k_{0}\right)}\left(\theta\left(1-e^{-\lambda x}\right)\right)^{k_{0}}}{C^{\left(k_{0}\right)}(\theta)}=\frac{\left(1-e^{-\lambda t}\right)^{k_{0}} a_{k_{0}}}{a_{k_{0}}}=\left(1-e^{-\lambda t}\right)^{k_{0}}$.

Este resultado é de esperar-se pois quando $\theta$ vai para $0^{+}, P\left(M=k_{0}\right)$ vai para 1 , assim, com probabilidade 1 , tem-se somente $k_{0}$ fatores de risco latentes e $T$ é o máximo de $k_{0}$ variáveis independentes com distribuição exponencial.

Proposição 3.2. A função de taxa de falha tem os limites seguintes:

$$
\lim _{t \rightarrow 0^{+}} h(t)=\frac{a_{1} \theta \lambda}{C(\theta)-a_{0}} \text { e } \lim _{t \rightarrow \infty} h(t)=\lambda .
$$

Prova. Segue do corolário 2.12 .

Segue deste resultado que para $t$ suficientemente grande a taxa de falha é aproximadamente constante, igual a $\lambda$, similarmente à distribuição exponencial. Além disto, $\lim _{t \rightarrow 0^{+}} h(t) \leq \lim _{t \rightarrow \infty} h(t)$ e, por tanto, a função de taxa de falha não pode ser uma função decrescente.

As proposições seguintes tratam a respeito da monotonia da função taxa de risco.

Proposição 3.3. A função de taxa de risco é crescente para t suficientemente grande.

Prova. Segue do lema 2.1.

Proposição 3.4. A função de taxa de falha é decrescente para $t$ suficientemente pequeno se, e somente se, $-a_{1} \sum_{m=2}^{\infty} a_{m} \theta^{m}+2 a_{2} \theta \sum_{m=1}^{\infty} a_{m} \theta^{m}<0$.

Prova. Segue do corolário 3.3

Então, a forma da gráfica da função de taxa de falha pode ser crescente, tipo banheira ou com múltiplos pontos críticos, mas não pode ser uma função de tipo decrescente. Infelizmente não tem-se achado condições que descrevam as possíveis formas. A função 
de taxa de falha é crescente para todos os casos particulares mostrados na Tabela 3.1 e descritos na Seção 3.4.

Os teoremas seguintes proporcionam expressões para obter os momentos e a média residual.

Proposição 3.5. Os momentos da distribuição ESPC são finitos e podem ser obtidas por

$$
E\left(T^{k}\right)=\frac{k !}{\lambda^{k} C(\theta)-a_{0}} \sum_{n=1}^{\infty} \sum_{j=1}^{n} \frac{1}{j^{k}} a_{n} \theta^{n}\left(\begin{array}{c}
n \\
j
\end{array}\right)(-1)^{j-1} .
$$

Prova. Desde que as distribuições série de potencias e exponencial tem seus momentos finitos, também resultam finitos os correspondentes a distribuição $E S P C$, como estabelecido no corolário 2.3. Para verificar (3.8), segue de (3.4) (que expressa a densidade $E S P C$ como uma mistura) que

$$
E\left(T^{k}\right)=\int_{0}^{\infty} t^{k} \sum_{m=1}^{\infty} \frac{a_{m} \theta^{m}}{C(\theta)-a_{0}} f_{m}(t ; \lambda) d t=\sum_{m=1}^{\infty} \frac{a_{m} \theta^{m}}{C(\theta)-a_{0}} \int_{0}^{\infty} t^{k} f_{m}(t ; \lambda) d t
$$

em que a troca na ordem entre a série e a integral pode ser feita pois os termos são positivos.

Por outra parte,

$$
\int_{0}^{\infty} t^{k} f_{m}(t ; \lambda) d t=\int_{0}^{\infty} t^{k} m \lambda e^{-\lambda t}\left(1-e^{-\lambda t}\right)^{m-1} d t=\int_{0}^{\infty} t^{k} m \lambda e^{-\lambda t} \sum_{i=0}^{m-1}\left(\begin{array}{c}
m-1 \\
i
\end{array}\right)(-1)^{i} e^{-\lambda i t} d t,
$$

em que a última igualdade é devida ao teorema do binômio; assim,

$$
\int_{0}^{\infty} t^{k} f(t ; \lambda) d t=\lambda \sum_{i=0}^{m-1} m\left(\begin{array}{c}
m-1 \\
i
\end{array}\right)(-1)^{i} \int_{0}^{\infty} t^{k} e^{-\lambda(i+1) t} d t=\frac{\Gamma(k+1)}{\lambda^{k}} \sum_{j=1}^{m} \frac{\left(\begin{array}{c}
m \\
j
\end{array}\right)(-1)^{j-1}}{j^{k}}
$$

e o resultado segue de (3.9).

Proposição 3.6. A média do tempo residual até o tempo de falha, dado o tempo de vida $t_{0}$, denotada por $m\left(t_{0}\right)=E\left(T-t_{0} \mid T \geq t_{0}\right)$, pode ser obtida na familia de distribuições ESPC como segue

$$
m\left(t_{0}\right)=\frac{1}{\lambda\left(C(\theta)-a_{0}\right) S\left(t_{0}\right)} \sum_{m=1}^{\infty} \sum_{j=1}^{m} \frac{1}{j}\left(\begin{array}{c}
m \\
j
\end{array}\right)(-1)^{j-1} e^{-\lambda j t_{0}} a_{m} \theta^{m} .
$$

Prova. $E\left(T-t_{0} \mid T \geq t_{0}\right)=\int_{0}^{\infty} t f(t) d t=\int_{T-t_{0} \mid T \geq t_{0}}^{\infty} \frac{f\left(t+t_{0}\right)}{S\left(t_{0}\right)} d t=\frac{1}{S\left(t_{0}\right)} \int_{0}^{\infty} t f_{T}\left(t+t_{0}\right) d t$;

então, a prova segue os mesmos passos da prova do teorema anterior. 


\subsection{Casos particulares}

Considerando os casos particulares da família de distribuições de série de potências Poisson (Cancho et al., 2011a), geométrica (Adamidis et al., 2005), logarítmica e binomial, como são descritos na Tabela 3.1, tem-se as distribuições exponencialbinomial (EPC), exponencial-geométrica (EGC), exponencial-logarítmica (ELC) e exponencial-binomial (EBC) complementares, respectivamente. A Tabela 3.4 mostra as funções de densidade e de taxa de risco para os casos particulares mencionados.

Tabela 3.2: Funções de densidade e taxa de risco para alguns casos particulares.

\begin{tabular}{ccc}
\hline Distribuição & Densidade & Taxa de risco \\
\hline Poisson & $\frac{\theta \lambda e^{-\lambda t-\theta e^{-\lambda t}}}{1-e^{-\theta}}$ & $\frac{\theta \lambda e^{-\lambda t}}{e^{\theta e^{-\lambda t}}-1}$ \\
Geométrica & $\frac{(1-\theta) \lambda e^{-\lambda t}}{\left(1-\theta\left(1-e^{-\lambda t}\right)\right)^{2}}$ & $\frac{(1-\theta) \lambda}{1-\theta\left(1-e^{-\lambda t}\right)}$ \\
Logarítmica & $\frac{-\theta \lambda e^{-\lambda t}}{\left(1-\theta\left(1-e^{-\lambda t}\right)\right) \log (1-\theta)}$ & $\frac{-\theta \lambda e^{-\lambda t}}{\left(1-\theta\left(1-e^{-\lambda t}\right)\right) \log \left(\frac{1-\theta}{1-\theta\left(1-e^{-\lambda t}\right)}\right)}$ \\
Binomial & $\frac{\theta \lambda e^{-\lambda t} n_{o}\left[1+\theta\left(1-e^{-\lambda t}\right)\right]^{n_{o}-1}}{(1+\theta)^{n_{o}}-1}$ & $\frac{\theta \lambda e^{-\lambda t} n_{o}\left[1+\theta\left(1-e^{-\lambda t}\right)\right]^{n_{o}-1}}{(1+\theta)^{n_{o}}-\left[1+\theta\left(1-e^{-\lambda t}\right)\right]^{n_{o}}}$ \\
\hline
\end{tabular}

As taxas de riscos para os casos particulares, Poisson, geométrica, logarítmica e binomial são monótonas crescentes e, como mostrado na Proposição 3.2, com limite igual a $\lambda$. Estas propriedades observam-se nos gráficos da figura 3.2, feitos considerando $\lambda=2$ e $\theta \in\{0,2,0,5,0,8\}$ e $\theta=0.6$ e $\lambda \in\{0,6,0,8,0,2\}$. Nos gráficos do modelo exponencial-binomial foi considerado $n_{o}=10$.

Na Tabela 3.3 são mostradas expressões para os momentos, média, variância e a média residual, obtidas de (3.8) e (3.10), para os casos particulares mencionados. 

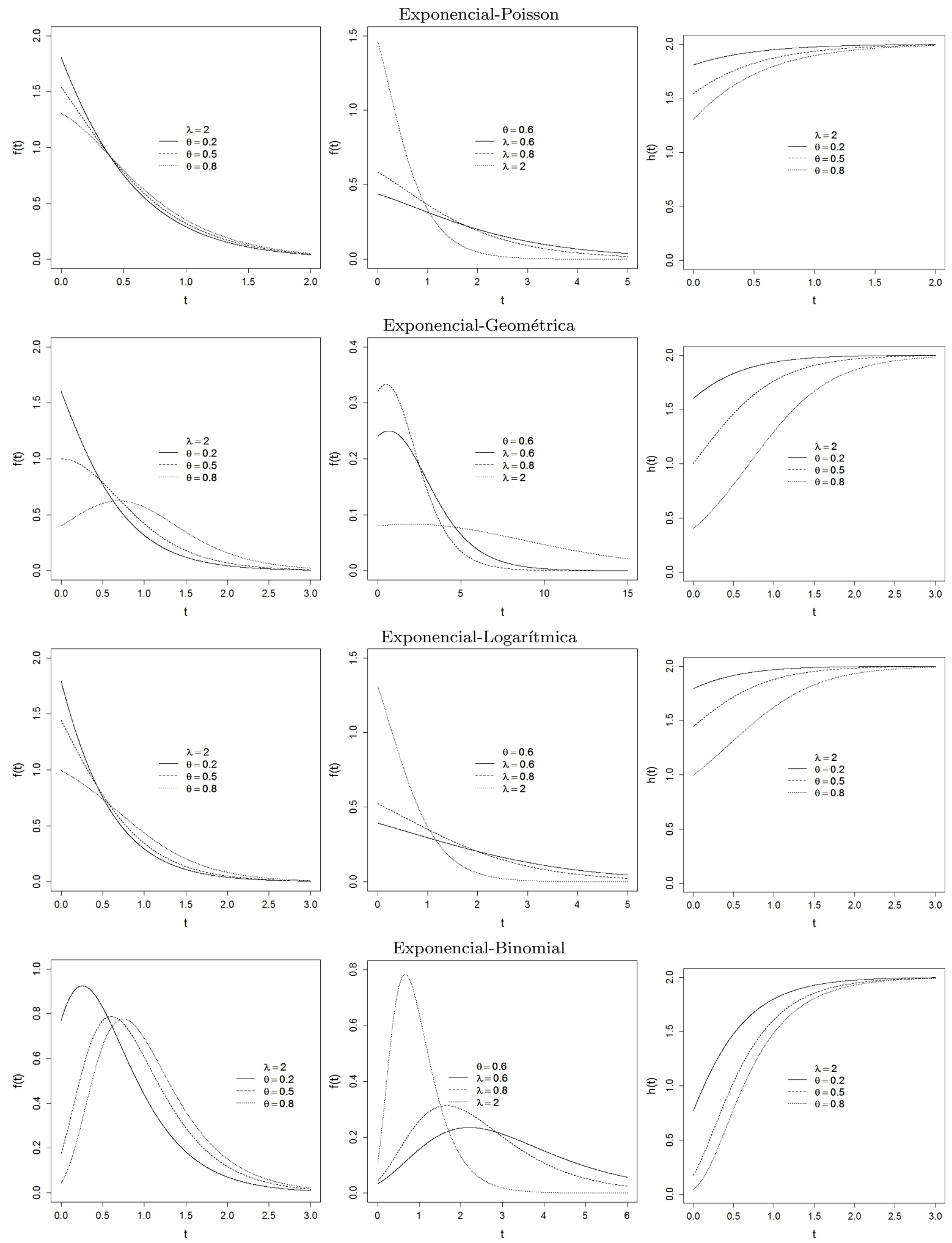

Figura 3.1: Gráficos das funções de densidade e taxa de risco para alguns casos particulares. 
Tabela 3.3: Momentos, média, variância e média residual para alguns casos particulares.

\begin{tabular}{l} 
Poisson \\
$E\left(T^{k}\right)=\frac{\theta k !}{\lambda^{k}\left(1-e^{-\theta}\right)} \sum_{j=0}^{\infty} \frac{(-\theta)^{j}}{(j+1)^{k+1} j !}=\frac{\theta k !}{\lambda^{k}\left(1-e^{-\theta}\right)}{ }_{k+1} F_{k+1}(1, \ldots, 1 ; 2, \ldots, 2 ;-\theta), \theta>0$ \\
$E(T)=\frac{\theta}{\lambda\left(1-e^{-\theta}\right)} \sum_{j=0}^{\infty} \frac{(-\theta)^{j}}{(j+1)^{2} j !}=\frac{\theta}{\lambda\left(1-e^{-\theta}\right)}{ }_{2} F_{2}(1,1 ; 2,2 ;-\theta)$ \\
$\operatorname{Var}(T)=\frac{2 \theta}{\lambda^{2}\left(1-e^{-\theta}\right)}\left[2{ }_{3} F_{3}(1,1,1 ; 2,2,2 ;-\theta)-\frac{\theta}{1-e^{-\theta e^{-\lambda t}}}{ }_{2} F_{2}^{2}(1,1 ; 2,2 ;-\theta)\right]$ \\
$m(t)=\frac{\theta e^{-\lambda t}}{\lambda\left(1-e^{-\theta}\right)} \sum_{j=0}^{\infty} \frac{\left(-\theta e^{-\lambda t}\right)^{j}}{(j+1)^{2} j !}=\frac{\theta e^{-\lambda t}}{\lambda\left(1-e^{-\theta e^{-\lambda t}}\right)}{ }_{2} F_{2}\left(1,1 ; 2,2 ;-\theta e^{-\lambda t}\right)$ \\
Geométrica \\
$E\left(T^{k}\right)=\frac{k !}{\lambda^{k} \theta} \sum_{j=1}^{\infty} \frac{(-1)^{j-1}}{j^{k}}\left(\frac{\theta}{1-\theta}\right)^{j}=\frac{k !}{\lambda^{k}(1-\theta)}{ }_{k+1} F_{k}\left(1, \ldots, 1 ; 2, \ldots, 2 ;-\frac{\theta}{1-\theta}\right), 0<\theta<1$ \\
$E(T)=-\frac{1}{\lambda \theta} \log (1-\theta)$ \\
$\operatorname{Var}(T)=\frac{1}{\lambda^{2}}\left[\frac{2}{1-\theta}{ }_{3} F_{2}\left(1,1,1 ; 2,2 ;-\frac{\theta}{1-\theta}\right)-\frac{1}{\theta^{2}} \log ^{2}(1-\theta)\right]$ \\
$m(t)=-\frac{1-\theta\left(1-e^{-\lambda t}\right)}{\lambda \theta e^{-\lambda t}}\left[\log (1-\theta)-\log \left(1-\theta\left(1-e^{-\lambda t}\right)\right)\right]$ \\
\hline
\end{tabular}

Logarítmica

$$
\begin{aligned}
& E\left(T^{k}\right)=-\frac{k !}{\lambda^{k} \log (1-\theta)} \sum_{j=1}^{\infty} \frac{(-1)^{j-1}}{j^{k+1}}\left(\frac{\theta}{1-\theta}\right)^{j}=-\frac{\theta k !}{\lambda^{k}(1-\theta) \log (1-\theta)}{ }_{k+2} F_{k+1}\left(1, \ldots, 1 ; 2, \ldots, 2 ;-\frac{\theta}{1-\theta}\right), 0<\theta<1 \\
& E(T)=-\frac{\theta}{\lambda(1-\theta) \log (1-\theta)} F_{2}\left(1,1,1 ; 2,2 ;-\frac{\theta}{1-\theta}\right) \\
& \operatorname{Var}(T)=-\frac{\theta}{\lambda^{2}(1-\theta) \log (1-\theta)}\left[2_{4} F_{3}\left(1,1,1,1 ; 2,2,2 ;-\frac{\theta}{1-\theta}\right)-\frac{\theta}{(1-\theta) \log (1-\theta)}{ }_{3} F_{2}^{2}\left(1,1,1 ; 2,2 ;-\frac{\theta}{1-\theta}\right)\right] \\
& m(t)=-\frac{\theta t}{\lambda(1-\theta)\left[\log (1-\theta)-\log \left(1-\theta\left(1-e^{-\lambda t}\right)\right)\right]_{3}} F_{2}\left(1,1,1 ; 2,2 ;-\frac{\theta e^{-\lambda t}}{1-\theta}\right)
\end{aligned}
$$

Binomial

$$
\begin{aligned}
& E\left(T^{k}\right)=\frac{k !(1+\theta)^{r}}{\lambda^{k}\left[(1+\theta)^{r}-1\right]} \sum_{j=1}^{r} \frac{(-1)^{j-1}}{j^{k}}\left(\begin{array}{l}
r \\
j
\end{array}\right)\left(\frac{\theta}{1+\theta}\right)^{j}, 0<\theta<1 \\
& E(T)=\frac{(1+\theta)^{r}}{\lambda\left[(1+\theta)^{r}-1\right]} \sum_{j=1}^{r} \frac{(-1)^{j-1}}{j}\left(\begin{array}{c}
r \\
j
\end{array}\right)\left(\frac{\theta}{1+\theta}\right)^{j} \\
& \operatorname{Var}(T)=\frac{(1+\theta)^{r}}{\lambda^{2}\left[(1+\theta)^{r}-1\right]}\left[2 \sum_{j=1}^{r} \frac{(-1)^{j-1}}{j^{2}}\left(\begin{array}{c}
r \\
j
\end{array}\right)\left(\frac{\theta}{1+\theta}\right)^{j}-\frac{(1+\theta)^{r}}{(1+\theta)^{r}-1}\left(\sum_{j=1}^{r} \frac{(-1)^{j-1}}{j}\left(\begin{array}{l}
r \\
j
\end{array}\right)\left(\frac{\theta}{1+\theta}\right)^{j}\right)^{2}\right] \\
& m(t)=\frac{(1+\theta)^{r}}{\lambda\left[(1+\theta)^{r}-\left(1+\theta\left(1-e^{-\lambda t}\right)\right)^{r}\right]} \sum_{j=1}^{r} \frac{(-1)^{j-1}}{j}\left(\begin{array}{l}
r \\
j
\end{array}\right)\left(\frac{\theta e^{-\lambda t}}{1+\theta}\right)^{j}
\end{aligned}
$$

em que $\underset{p}{F}\left(a_{1}, \ldots, a_{p} ; b_{1}, \ldots, b_{q} ; z\right)=\sum_{n=1}^{\infty} \frac{\left(a_{1}\right)_{n} \ldots\left(a_{p}\right)_{n}}{\left(b_{1}\right)_{n} \ldots\left(b_{q}\right)_{n}} \frac{z^{n}}{n !}$ é a função hipergeométrica generalizada e $(a)_{n}=\frac{\Gamma(a+n)}{\Gamma(a)}$ é o símbolo de Pochhammer; esta função converge para $z \in \mathbb{R}$, se $p<q+1$, converge para $z \in(-1,1)$, se $p=q+1$ e diverge para $x \neq 0$, se $p>q=1$, como citado em Johnson et al. (2005, p. 27). 


\subsection{Inferência}

\subsubsection{Estimação por máxima verossimilhança}

A função de log-verossimilhança, baseada nas observações $\left(t_{1}, \ldots, t_{n}\right)$ de uma amostra aleatória da distribuição exponencial-série de potência com parâmetros $\theta$ e $\lambda$, está dada por

$$
\ell(\theta, \lambda)=n \log (\theta \lambda)-\lambda \sum_{i=1}^{n} t_{i}+\sum_{i=1}^{n} \log \left(C^{\prime}\left(\theta\left(1-e^{-\lambda t_{i}}\right)\right)\right)-n \log (C(\theta)) .
$$

As estimativas de máxima verossimilhança dos parâmetros $\theta$ e $\lambda$ podem ser obtidas maximizando diretamente a função de log-verossimilhança (3.11) ou, alternativamente, achando a solução do sistema de equações não-lineares seguinte

$$
\begin{aligned}
& \frac{\partial \ell(\theta, \lambda)}{\partial \theta}=\frac{n}{\theta}-\frac{n C^{\prime}(\theta)}{C(\theta)}+\sum_{i=1}^{n} \frac{\left(1-e^{-\lambda t_{i}}\right) C^{\prime \prime}\left(\theta\left(1-e^{-\lambda t_{i}}\right)\right)}{C^{\prime}\left(\theta\left(1-e^{-\lambda t_{i}}\right)\right)}=0, \\
& \frac{\partial \ell(\theta, \lambda)}{\partial \lambda}=\frac{n}{\lambda}-\sum_{i=1}^{n} t_{i}+\theta \sum_{i=1}^{n} \frac{t_{i} e^{-\lambda t_{i}} C^{\prime \prime}\left(\theta\left(1-e^{-\lambda t_{i}}\right)\right)}{C^{\prime}\left(\theta\left(1-e^{-\lambda t_{i}}\right)\right)}=0,
\end{aligned}
$$

em que $C^{\prime \prime}\left(\theta\left(1-e^{-\lambda t_{i}}\right)\right)$ é a segunda derivada de $C(\theta)$ respeito a $\theta$ avaliada em $\theta\left(1-e^{-\lambda t_{i}}\right)$.

\subsubsection{Estimação por intervalos}

Inferência para os parâmetros, com amostras grandes, pode ser feita com os estimadores de máxima verossimilhança. Segundo Cox \& Hinkley (1974), sob determinadas condições de regularidade, pode-se mostrar que

$$
\sqrt{n}(\widehat{\theta}-\theta, \widehat{\lambda}-\lambda) \rightarrow N_{2}\left(\mathbf{0}, \boldsymbol{I}_{(\theta, \lambda)}^{-1}\right),
$$

em que $\boldsymbol{I}(\theta, \lambda)$ é a matriz de informação de Fisher, isto é,

$$
\boldsymbol{I}(\theta, \lambda)=-\left[\begin{array}{cc}
E\left(\frac{\partial^{2} L(\theta, \lambda ; T)}{\partial \theta^{2}}\right) & E\left(\frac{\partial^{2} L(\theta, \lambda ; T)}{\partial \theta \partial \lambda}\right) \\
E\left(\frac{\partial^{2} L(\theta, \lambda ; T)}{\partial \theta \partial \lambda}\right) & E\left(\frac{\partial^{2} L(\theta, \lambda ; T)}{\partial \lambda^{2}}\right)
\end{array}\right],
$$


com $L(\theta, \lambda ; t)=\log (f(t))$, o logaritmo da densidade da distribuição $\operatorname{ESPC}(\theta, \lambda)$ (dado em (3.3)). As segundas derivadas de $L$ são

$$
\begin{aligned}
& \frac{\partial^{2} L(\theta, \lambda ; t)}{\partial \theta^{2}}=-\frac{1}{\theta^{2}}-\frac{C^{\prime \prime}(\theta) C(\theta)-C^{\prime^{2}}(\theta)}{C^{2}(\theta)}+\frac{b^{2}(t)\left[C^{\prime \prime \prime}(\theta b(t)) C^{\prime}(\theta b(t))-C^{\prime^{2}}(\theta b(t))\right]}{C^{\prime^{2}}(\theta b(t))}, \\
& \frac{\partial^{2} L(\theta, \lambda ; t)}{\partial \theta \partial \lambda}=\frac{t e^{-\lambda t}}{C^{\prime^{2}}(\theta b(t))}\left\{C^{\prime}(\theta b(t))\left[C^{\prime \prime}(\theta b(t))+\theta b(t) C^{\prime \prime \prime}(\theta b(t))\right]-\theta b(t) C^{\prime^{2}}(\theta b(t))\right\}, \\
& \frac{\partial^{2} L(\theta, \lambda ; t)}{\partial \lambda^{2}}=-\frac{1}{\lambda^{2}}-\frac{\theta t^{2} e^{-\lambda t}}{C^{\prime 2}(\theta b(t))}\left\{C^{\prime}(\theta b(t))\left[C^{\prime \prime}(\theta b(t))-\theta e^{-\lambda t} C^{\prime \prime \prime}(\theta b(t))\right]+\theta e^{-\lambda t} C^{\prime \prime 2}(\theta b(t))\right\},
\end{aligned}
$$

em que $b(t)=1-e^{-\lambda t}$.

\subsection{Estudo de simulação}

Esta seção apresenta os resultados de um estudo de simulação, baseado em 10000 amostras simuladas de tamanhos $n=20,30,50,100$, e 200, para o modelo binomial exponencial (com $n_{0}=13$ ). Desde que são conhecidas as propriedades assintóticas dos estimadores de máxima verossimilhança, o estudo considera tamanhos amostrais não muito grandes. Na Tabela 3.4 são mostrados os valores médios das estimativas, seus erros quadráticos médios (emc) e suas variâncias e covariâncias. Também são mostradas as variâncias e covariâncias assintóticas, determinadas pela informação de Fisher; além disto, outras duas estimativas foram obtidas para as variâncias e covariâncias, uma estimando a informação de Fisher com as estimativas dos parâmetros obtidas e uma outra com a matriz de informação de Fisher observada. Observa-se que, ainda com tamanho de amostra pequeno, as estimativas dos parâmetros são boas para valores de $\theta$ pequenos e todos os valores de $\lambda$ considerados. Como esperado estas estimativas, tornam-se bastante precisas conforme $n$ aumenta. Para tamanhos amostrais pequenos as variâncias e covariâncias dos estimadores estimadas com as informações de Fisher exata, estimada e observada somente sao bem estimadas quando $\theta$ é pequeno; mas, como esperado, estas quantidades tornam-se próximas conforme o tamanho amostral aumenta. As variâncias e covariânzas estimadas com as informações de Fisher estimada e observada são muito próximas, ainda com mostras pequenas, do que as correspondentes à informação de Fisher esperada, como mencionado em Efron \& Hinkley (1978). O estudo de simulação também incluiu a probabilidade de cobertura dos intervalos de confiança para parâmetros de $\theta$ e $\lambda$, considerando duas vezes o erro padrão de estimação obtido com a informação de Fisher observada, obtendose probabilidades grandes devido às relativas boas precisões das estimativas, como mostrado nas duas ultimas colunas da Tabela 3.4. 
Tabela 3.4: Média das variâncias e covariâncias das EMV, erro quadrático meio das EMV e probabilidades de cobertura dos intervalos do $95 \%$ de confiança para os parâmetros.

\begin{tabular}{|c|c|c|c|c|c|c|c|c|c|c|c|c|c|c|c|c|}
\hline \multirow[b]{2}{*}{$n$} & \multirow[b]{2}{*}{$(\theta ; \lambda)$} & \multirow[b]{2}{*}{$\hat{\theta}$} & \multirow[b]{2}{*}{$\hat{\lambda}$} & \multicolumn{5}{|c|}{ Simulados } & \multicolumn{3}{|c|}{ Informação esperada } & \multicolumn{3}{|c|}{ informação observada } & \multicolumn{2}{|c|}{ Cobertura } \\
\hline & & & & $\operatorname{Var}(\hat{\theta})$ & $\operatorname{Var}(\hat{\lambda})$ & $m s e(\hat{\theta})$ & $m s e(\hat{\lambda})$ & $\operatorname{Cov}(\hat{\theta}, \hat{\lambda})$ & $\overline{\operatorname{Var}(\hat{\theta})}$ & $\operatorname{Var}(\hat{\lambda})$ & $\operatorname{Cov}(\hat{\theta}, \hat{\lambda})$ & $\overline{\operatorname{Var}(\hat{\theta})}$ & $\operatorname{Var}(\hat{\lambda})$ & $\operatorname{Cov}(\hat{\theta}, \hat{\lambda})$ & $\theta$ & $\lambda$ \\
\hline \multirow[t]{5}{*}{20} & $(0,1 ; 2)$ & 151 & 2.228 & 0.020 & 0.420 & 0.022 & 0.472 & 0.063 & 0.015 & 0.401 & 0.061 & 0.022 & 0.447 & 0.071 & 0.999 & 0.953 \\
\hline & $(0,3 ; 2)$ & 390 & 2.128 & .051 & 0.221 & 0.059 & 0.237 & 0.073 & .026 & 0.198 & 0.053 & 0.065 & 0.220 & 0.077 & 0.985 & 0.955 \\
\hline & $(0,5 ; 2)$ & 609 & 2.078 & .069 & 0.142 & 0.081 & 0.148 & 0.064 & 0.063 & 0.148 & 0.070 & 0.143 & 0.160 & 0.100 & .964 & 0.968 \\
\hline & $(0,7 ; 2)$ & 0.763 & 2.032 & 0.057 & 0.099 & 0.061 & 0.100 & 0.043 & .145 & 0.131 & 0.103 & 0.209 & 0.133 & 0.115 & 0.956 & 0.978 \\
\hline & $(0,9 ; 2)$ & 0.857 & 1.979 & 0.039 & 0.071 & 0.041 & 0.072 & 0.026 & 0.305 & 0.125 & 0.149 & 0.248 & 0.115 & 0.118 & 0.953 & 0.982 \\
\hline \multirow[t]{5}{*}{30} & $(0,1 ; 2)$ & 0.129 & 2.140 & 0.011 & 0.261 & 0.012 & 0.280 & 0.038 & 0.010 & 0.268 & 0.041 & 0.012 & 0.290 & 0.045 & 0.998 & 0.958 \\
\hline & $(0,3 ; 2)$ & 0.356 & 2.079 & 0.031 & 0.151 & 0.034 & 0.157 & 0.049 & 0.018 & 0.132 & 0.035 & 0.032 & 0.142 & 0.045 & 0.978 & 0.954 \\
\hline & $(0,5 ; 2)$ & 0.587 & 2.052 & 0.053 & 0.099 & 0.061 & 0.102 & 0.049 & 0.042 & 0.099 & 0.047 & 0.085 & 0.104 & 0.064 & 0.964 & 0.960 \\
\hline & $(0,7 ; 2)$ & 0.760 & 2.024 & 0.049 & 0.069 & 0.053 & 0.070 & 0.036 & 0.097 & 0.088 & 0.068 & 0.140 & 0.088 & 0.078 & 0.954 & 0.976 \\
\hline & $(0,9 ; 2)$ & 0.861 & 1.978 & 0.033 & 0.049 & 0.034 & 0.050 & 0.022 & 0.203 & 0.083 & 0.099 & 0.172 & 0.077 & 0.082 & 0.953 & 0.979 \\
\hline \multirow[t]{5}{*}{50} & $(0,1 ; 2)$ & 118 & 2.084 & 0.006 & 0.162 & 0.006 & 0.169 & 0.023 & 0.006 & 0.161 & 0.025 & 0.007 & 0.167 & 0.026 & 0.986 & 0.955 \\
\hline & $(0,3 ; 2)$ & 333 & 2.050 & 0.015 & 0.087 & 0.017 & 0.090 & 0.027 & 0.011 & 0.079 & 0.021 & 0.015 & 0.083 & 0.024 & 0.975 & 0.949 \\
\hline & $(0,5 ; 2)$ & 0.558 & 2.037 & 0.035 & 0.062 & 0.038 & 0.063 & 0.033 & 0.025 & 0.059 & 0.028 & 0.043 & 0.062 & 0.035 & 0.966 & 0.962 \\
\hline & $(0,7 ; 2)$ & 0.756 & 2.027 & 0.037 & 0.043 & 0.040 & 0.044 & 0.026 & 058 & 0.053 & 0.041 & 0.083 & 0.053 & 47 & 0.963 & 0.976 \\
\hline & $(0,9 ; 2)$ & 0.874 & 1.980 & 0.025 & 0.030 & 0.026 & 0.030 & 0. & 22 & 0.050 & 0.060 & 0.110 & 0.047 & 152 & 0.957 & 0.979 \\
\hline \multirow[t]{5}{*}{100} & $(0,1 ; 2)$ & 107 & 2.035 & .003 & 0.078 & 0.003 & 0.079 & 0.012 & 003 & 0.080 & 0.012 & 0.003 & 0.082 & 0.013 & 0.980 & 0.962 \\
\hline & $(0,3 ; 2)$ & 315 & 2.023 & 0.006 & 0.041 & 0.006 & 0.041 & 0.011 & .005 & 0.040 & 0.011 & 0.006 & 0.040 & 0.011 & 0.966 & 0.956 \\
\hline & $(0,5 ; 2)$ & 0.529 & 2.022 & 0.017 & 0.031 & 0.017 & 0.032 & 0.016 & 0.013 & 0.030 & 0.014 & 0.017 & 0.030 & 0.016 & 0.966 & 0.954 \\
\hline & $(0,7 ; 2)$ & 0.741 & 2.018 & 0.026 & 0.024 & 0.028 & 0.024 & 0.017 & 0.029 & 0.026 & 0.021 & 0.039 & 0.027 & 0.023 & 0.961 & 0.969 \\
\hline & $(0,9 ; 2)$ & 0.882 & 1.985 & 0.018 & 0.016 & 0.018 & 0.016 & 0.010 & 0.061 & 0.025 & 0.030 & 0.057 & 0.024 & 0.027 & 0.960 & 0.976 \\
\hline \multirow[t]{5}{*}{200} & $(0.1 ; 2)$ & .102 & 2.016 & 0.002 & 0.042 & 0.002 & 0.042 & 0.006 & 0.001 & 0.040 & 0.006 & 0.002 & 0.041 & 0.006 & 0.961 & 0.956 \\
\hline & $(0,3 ; 2)$ & 0.307 & 2.010 & 0.003 & 0.020 & 0.003 & 0.020 & 0.006 & .003 & 0.020 & 0.005 & 0.003 & 0.020 & 0.005 & 0.962 & 0.956 \\
\hline & $(0,5 ; 2)$ & 0.518 & 2.012 & 0.008 & 0.015 & 0.008 & 0.015 & 0.008 & .006 & 0.015 & 0.007 & 0.007 & 0.015 & 0.008 & 0.962 & 0.956 \\
\hline & $(0,7 ; 2)$ & 0.728 & 2.015 & 0.015 & 0.013 & 0.016 & 0.013 & 0.010 & 0.015 & 0.013 & 0.010 & 0.018 & 0.013 & 0.011 & 0.965 & 0.965 \\
\hline & $(0,9 ; 2)$ & 0.891 & 1.993 & 0.012 & 0.009 & 0.012 & 0.009 & 0.007 & 0.031 & 0.012 & 0.015 & 0.030 & 0.012 & 0.014 & 0.963 & 0.979 \\
\hline \multirow[t]{5}{*}{20} & ;6) & 149 & 6.666 & 018 & 677 & 0.020 & 4.120 & 0 & 015 & 613 & 0.184 & 0.021 & 3.985 & 0 & .999 & 0.961 \\
\hline & $(0,3 ; 6)$ & 385 & 6.353 & 0.051 & 2.010 & 0.058 & 2.134 & 0.217 & .026 & 1.781 & 0.158 & 0.065 & 1.987 & 0.231 & 0.981 & 0.958 \\
\hline & $(0,5 ; 6)$ & 0.615 & 6.248 & 0.069 & 1.256 & 0.082 & 1.317 & 0.188 & 0.063 & 1.334 & 0.210 & 0.147 & 1.448 & 0.307 & 0.969 & 0.970 \\
\hline & $(0,7 ; 6)$ & 0.771 & 6.111 & 0.056 & 0.883 & 0.061 & 0.895 & 0.130 & . 145 & 1.182 & 0.308 & 0.212 & 1.191 & 0.346 & 0.958 & 0.980 \\
\hline & $(0,9 ; 6)$ & 0.854 & 5.943 & 0.040 & 0.661 & 0.042 & 0.664 & 0.085 & 0.305 & 1.123 & 0.447 & 0.246 & 1.035 & 0.352 & 0.946 & 0.980 \\
\hline \multirow[t]{5}{*}{30} & $(0,1 ; 6)$ & 128 & 6.411 & 11 & 2.348 & 0.012 & 2.516 & 0 & 10 & 2.408 & 0 . & 12 & 2.595 & 34 & 0.999 & 0.965 \\
\hline & $(0,3 ; 6)$ & 0.358 & 6.243 & 32 & 1.317 & 0.035 & 1.376 & 0.1 & 018 & 1.187 & 0.105 & 0.033 & 1.274 & 36 & 0.980 & 0.951 \\
\hline & $(0,5 ; 6)$ & 0.594 & 6.185 & 0.054 & 0.882 & 0.063 & 0.916 & 0.147 & 0.042 & 0.889 & 0.140 & 0.088 & 0.946 & 0.195 & 0.967 & 0.966 \\
\hline & $(0,7 ; 6)$ & 0.761 & 6.081 & 0.048 & 0.598 & 0.052 & 0.605 & 0.106 & 0.097 & 0.788 & 0.205 & 0.140 & 0.797 & 0.234 & 0.961 & 0.978 \\
\hline & $(0,9 ; 6)$ & 0.862 & 5.924 & 0.033 & 0.429 & 0.034 & 0.435 & 0.063 & 0.203 & 0.749 & 0.298 & 0.172 & 0.691 & 0.245 & 0.954 & 0.982 \\
\hline 50 & $(0,1 ; 6)$ & 0.115 & 6.248 & 0.006 & 1.420 & 0.006 & 1.481 & 0.069 & 0.006 & 1.445 & 0.074 & 0.007 & 1.532 & 0.078 & 0.986 & 0.960 \\
\hline & $(0,3 ; 6)$ & 0.332 & 6.161 & 0.015 & 0.779 & 0.016 & 0.805 & 0.077 & 0.011 & 0.712 & 0.063 & 0.015 & 0.748 & 0.073 & 0.976 & 0.953 \\
\hline & $(0,5 ; 6)$ & 0.559 & 6.123 & 0.035 & 0.531 & 0.038 & 0.546 & 0.094 & 0.025 & 0.533 & 0.084 & 0.043 & 0.559 & 0.106 & 0.968 & 0.961 \\
\hline & $(0,7 ; 6)$ & 0.755 & 6.070 & 0.038 & 0.388 & 0.041 & 0.392 & 0.079 & .058 & 0.473 & 0.123 & 0.083 & 0.479 & 0.142 & 0.958 & 0.974 \\
\hline & $(0,9 ; 6)$ & 0.869 & 5.938 & 0.026 & 0.269 & 0.027 & 0.273 & 0.047 & 0.122 & 0.449 & 0.179 & 0.108 & 0.423 & 0.155 & 0.951 & 0.980 \\
\hline 100 & $(0,1 ; 6)$ & 0.106 & 6.099 & 03 & 0.750 & 0.003 & 0.760 & & 03 & 0.723 & $0 .($ & 0.003 & 0.745 & & 0.981 & 0.958 \\
\hline & $(0,3 ; 6)$ & 0.314 & 6.074 & .006 & 0.374 & 0.006 & 0.379 & & 0.005 & 0.356 & 0.032 & 0.006 & 0.365 & 0.034 & 0.967 & 0.953 \\
\hline & $(0,5 ; 6)$ & 0.531 & 6.072 & 0.016 & 0.269 & 0.017 & 0.274 & 0.047 & 0.013 & 0.267 & 0.042 & 0.017 & 0.274 & 0.048 & 0.972 & 0.956 \\
\hline & $(0,7 ; 6)$ & 0.738 & 6.043 & 0.025 & 0.212 & 0.026 & 0.214 & 0.050 & 0.029 & 0.236 & 0.062 & 0.038 & 0.240 & 0.069 & 0.963 & 0.969 \\
\hline & $(0,9 ; 6)$ & 0.880 & 5.959 & 0.018 & 0.152 & 0.018 & 0.153 & 0.032 & 0.061 & 0.225 & 0.089 & 0.057 & 0.216 & 0.082 & 0.961 & 0.979 \\
\hline 200 & $(0,1 ; 6)$ & 0.104 & 6.058 & 0.002 & 0.366 & 0.002 & 0.369 & 0.019 & 0.001 & 0.361 & 0.018 & 0.002 & 0.367 & 0.019 & 0.957 & 0.955 \\
\hline & $(0,3 ; 6)$ & 0.307 & 6.039 & 0.003 & 0.183 & 0.003 & 0.184 & 0.016 & 0.003 & 0.178 & 0.016 & 0.003 & 0.181 & 0.016 & 0.962 & 0.954 \\
\hline & $(0,5 ; 6)$ & 0.515 & 6.040 & 0.007 & 0.133 & 0.008 & 0.134 & 0.023 & 0.006 & 0.133 & 0.021 & 0.007 & 0.135 & 0.022 & 0.965 & 0.958 \\
\hline & $(0,7 ; 6)$ & 0.726 & 6.035 & 0.016 & 0.120 & 0.016 & 0.121 & 0.032 & 0.015 & 0.118 & 0.031 & 0.018 & 0.120 & 0.034 & 0.963 & 0.959 \\
\hline & $(0,9 ; 6)$ & 0.893 & 5.981 & 0.012 & 0.076 & 0.012 & 0.077 & 0.020 & 0.031 & 0.112 & 0.045 & 0.030 & 0.110 & 0.043 & 0.962 & 0.960 \\
\hline
\end{tabular}

\subsection{Aplicação}

Serão analisados os dados extraídos de Lawless (2003b), também estudados por Gupta \& Kundu (1999), Gupta \& Kundu (2003) e Cancho et al. (2011a), que correspondem ao número de milhões de rotações antes da falha para cada um dos rolamentos de 23 esferas em um teste de resistência de rolamentos. Como foi feito nos estudos mencionados, em primeiro lugar determina-se a possível forma da taxa de falhas 
dos dados, mediante o gráfico TTT plot (Aarset (1985)) determinado pelos pontos $\left(\frac{r}{n}, G\left(\frac{r}{n}\right)\right), \operatorname{com} G\left(\frac{r}{n}\right)=\left[\sum_{i=1}^{r} Y_{(i)}+(n-r) Y_{(r)}\right] /\left(\sum_{i=1}^{n} Y_{(i)}\right), r=1, \ldots, n$, em que $Y_{(i)}$ é a estatística de ordem $i$ da amostra. Demonstra-se que a função de taxa de falha é crescente (decrescente) se o TTT plot é côncavo (convexo). Embora, o TTT plot seja uma condição suficiente, mas não uma condição necessária para indicar a forma de função de taxa de falha, ele é usado aqui como um indicativo bruto de sua forma. A seguinte figura mostra o TTT plot para os dados considerados

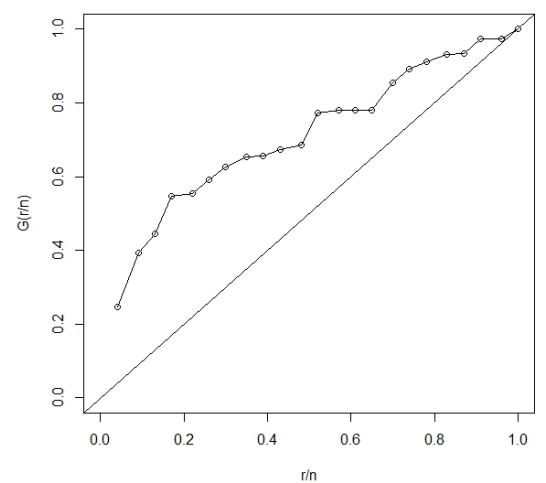

Figura 3.2: Gráfico TTT plot.

O gráfico é côncavo indicando uma função de taxa de falhas crescente, que pode ser adequadamente ajustado por uma distribuição exponencial-série de potências complementar; então, propomos como candidatos os casos particulares Poisson, Geométrica, Logarítmica e Binomial e determinaremos aquele que melhor ajusta os dados. Consideramos também o modelo Weibull, pois, é um modelo com taxa de risco monótona. Para o modelo binomial foi usado $n_{0}=5$ (valor que determina o modelo exponencial-binomial com maior verossimilhança avaliada nas estimativas dos parâmetros, considerando $n_{0} \leq 100$ ).

A seguir será determinado o modelo que melhor ajuste os dados. São obtidas as estimativas de máxima verossimilhança, dos parâmetros $\theta$ e $\lambda$, usando (3.11) e a função BBoptim do programa R (R Development Core Team, 2011). A Tabela 3.5 mostra os valores máximos da função log-verossimilhança, $l($.$) , com cada modelo ajustado, o$ correspondente coeficiente AIC $(-2 l(\hat{\theta}, \hat{\lambda})+2 p$, em que $p$ é o número de parâmetros estimados), a estatística $\chi^{2}$ (com a distribuição de freqüências da Tabela 3.6 como em Gupta \& Kundu (1999) e Gupta \& Kundu (2003)) e a estatística de KolmogorovSmirnov (K-S) (com seu valor $p$ obtido com o programa dado em Marsaglia et al. (2003)). 
Tabela 3.5: Valores de $l($.$) , AIC, estatísticas \chi^{2}$ e K-S para as distribuições ajustadas.

\begin{tabular}{ccccc}
\hline Distribution & log-ver & AIC & $\chi^{2}$ (valor p) & K-S(valor p) \\
\hline Binomial & $-112,9874$ & 229,9748 & $0,7117(0,8704)$ & $0,1061(0,9339)$ \\
Poisson & $-113,1521$ & 230,3042 & $0,8630(0,8344)$ & $0,1150(0,8875)$ \\
Geométrica & $-114,3502$ & 232,7004 & $2,4302(0,4880)$ & $0,1387(0,7173)$ \\
Logarítmica & $-116,7022$ & 237,4044 & $6,5446(0.0879)$ & $0,2066(0,2441)$ \\
Weibull & $-113,6887$ & 231,3774 & $2,4634(0,4819)$ & $0,1512(0,6157)$ \\
\hline
\end{tabular}

Tabela 3.6: Frequências observadas e esperadas para os modelos ajustados.

\begin{tabular}{ccccccc}
\hline Intervalo & Observada & Binomial & Poisson & Geométrica & Logarítmica & Weibull \\
\hline $0-40$ & 3 & 40,419 & 39,909 & 0,4203 & 79,133 & 80,281 \\
$40-80$ & 12 & 111,316 & 111,661 & 24,957 & 72,075 & 78,219 \\
$80-120$ & 5 & 55,674 & 56,672 & 86,176 & 50,636 & 43,296 \\
$120-160$ & 2 & 16,704 & 16,375 & 85,395 & 21,211 & 18,637 \\
$160-200$ & 1 & 0,4393 & 0,4089 & 2.4442 & 0,5499 & 0,6712 \\
\hline
\end{tabular}

Então, a maior log-verossimilhança, ou menor AIC, a menor estatística qui-quadrado e a menor estatística K-S correspondem ao modelo exponencial-binomial.

A seguinte figura mostra as densidades das distribuições ajustadas sobrepostas ao histograma dos dados e também as funções de sobrevivência ajustadas sobrepostas à curva de Kaplan-Meir.
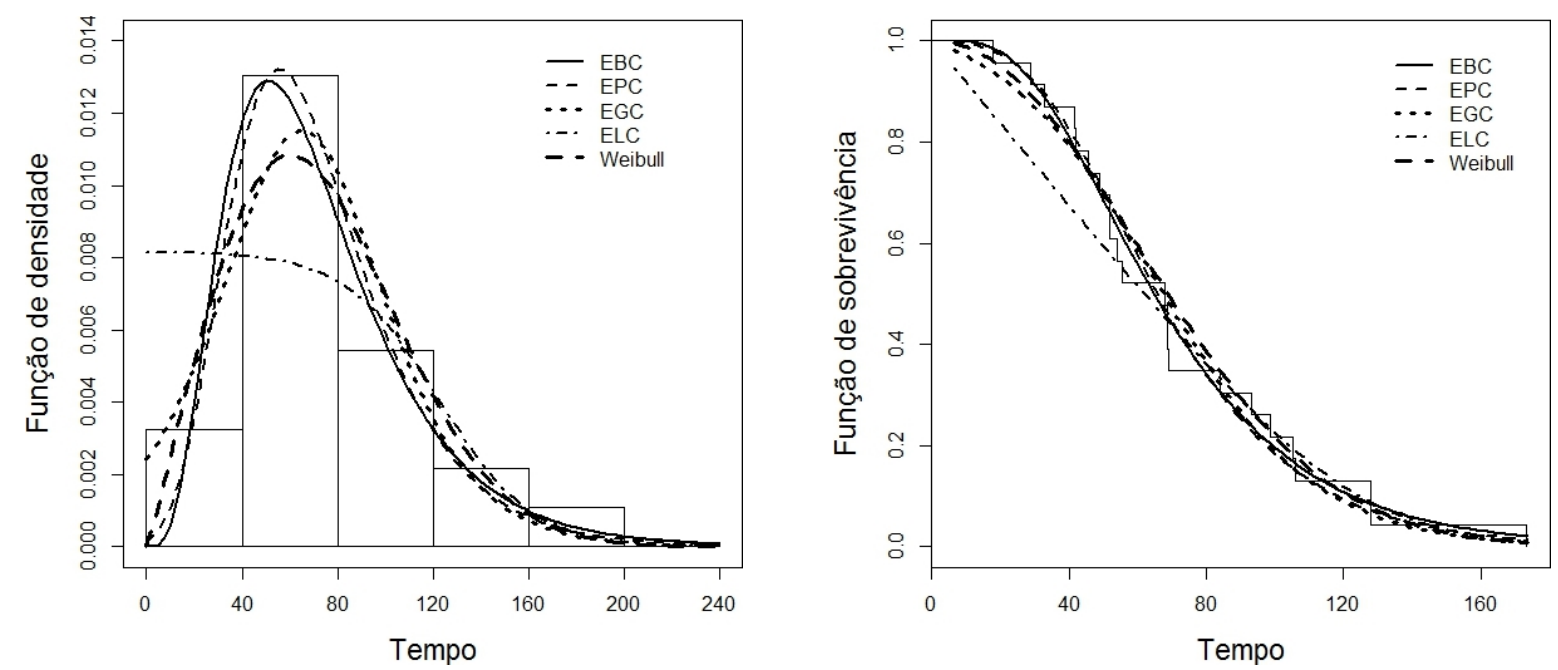

Figura 3.3: Esquerda: densidades das distribuições ajustadas sobrepostas ao histograma dos dados. Direita: funções de sobrevivência ajustadas sobrepostas à curva de Kaplan-Meir. 
Na Tabela 3.7 são mostradas as estimativas dos parâmetros. Como a estimativa do parâmetro $\theta$ não é pequena, o estudo de simulação feita na Seção 3.6 sugere que as estimativas do erro padrão associado obtida com a informação de Fisher observada não é recomendável; portanto, foi utilizado simulação bootstrap para obter estimativas dos erros padrões de estimação e intervalos de confiança. Os resultados também são mostrados na Tabela 3.7.

Tabela 3.7: Resumo das estimativas

\begin{tabular}{cccc}
\hline Parâmetro & Estimativa & Desvio padrão & Intervalo do 95\% de confiança \\
\hline$\theta$ & 0,9999 & 0,1683 & $(0,4010 ; 1)$ \\
$\lambda$ & 0,0346 & 0,0042 & $(0,0277 ; 0,0452)$ \\
\hline
\end{tabular}

Os histogramas dos valores das estimativas geradas mostram uma falta de normalidade, confirmando o uso do método bootstrap, como visto na Figura 3.4.
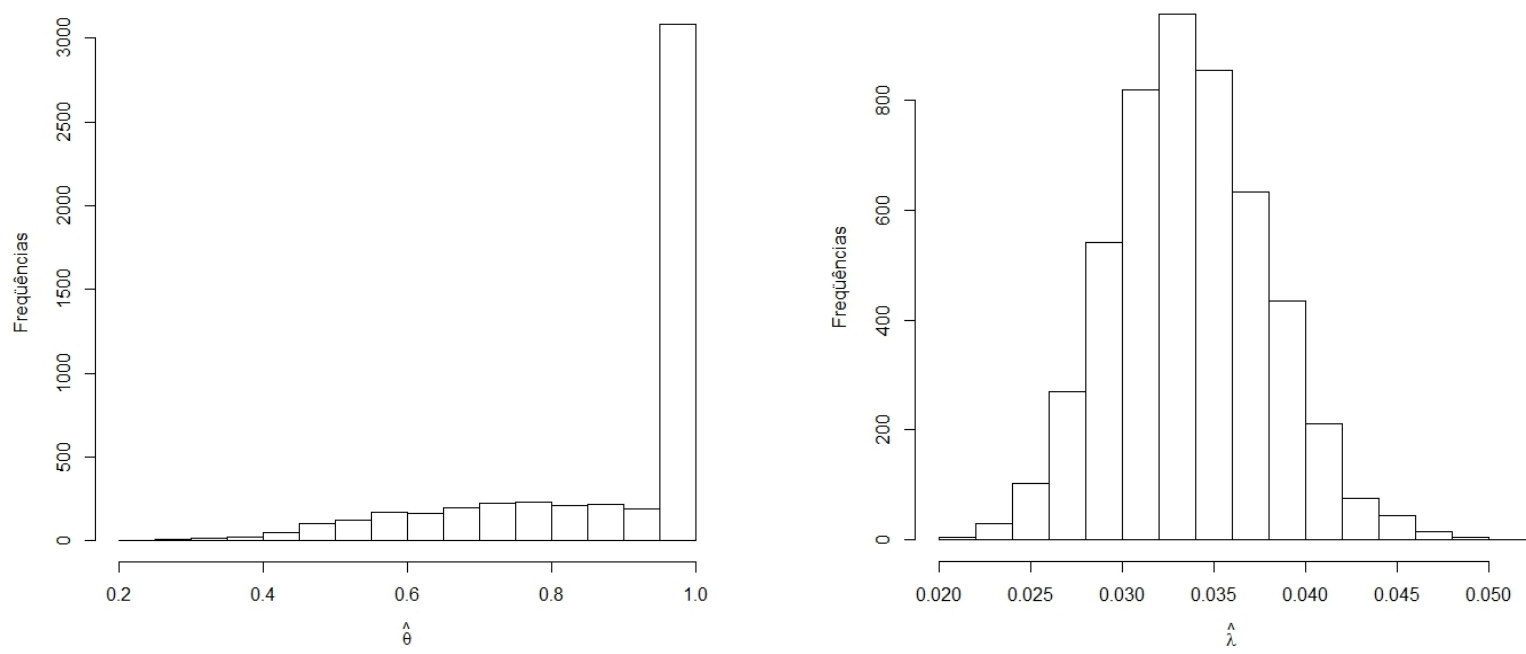

Figura 3.4: Histogramas das réplicas bootstrap. Direita: de $\hat{\theta}$. Esquerda: de $\hat{\lambda}$. 


\section{Capítulo 4}

\section{Um modelo com fração de cura: exponencial generalizado-série de potências com covariáveis}

\subsection{Dedução do modelo}

Além das suposições consideradas no modelo de fatores latentes no Capítulo 2, considere-se que o número de fatores latentes, $M$, segue uma distribuição de série de potências, mas agora não truncada no zero para obter um modelo com fração de cura. Segue de (2.28) que a função de distribuição de probabilidades de $M$ é dada por

$$
f_{M}(m)=P(M=m)=\frac{a_{m} \theta^{m}}{C(\theta)}, m \in\{0,1,2, \ldots\} ; \theta \in \Theta,
$$

em que a sequência de números $a_{0}, a_{1}, \ldots$, o conjunto $\Theta$ e a função série de potências $C(\theta)=\sum_{m=0}^{\infty} a_{m} \theta^{m}, \forall \theta \in \Theta$, foram descritas em (2.28). Alguns casos particulares são mostrados na Tabela 4.1.

Tabela 4.1: Casos particulares da distribuição de série de potências

\begin{tabular}{llcccc} 
Distribuição & $f_{M}(m)$ & $a_{m}$ & $C(\theta)$ & $\Theta$ & $p_{0}$ \\
\hline Poisson & $\frac{e^{-\theta} \theta^{m}}{m !}$ & $\frac{1}{m !}$ & $e^{\theta}$ & $(0, \infty)$ & $e^{-\theta}$ \\
Geometrica & $\theta^{m}(1-\theta)$ & 1 & $(1-\theta)^{-1}$ & $(0,1)$ & $1-\theta$ \\
Logarítmica & $\frac{\theta^{m+1}}{-(m+1) \log (1-\theta)}$ & $\frac{1}{m}$ & $-\log (1-\theta)$ & $(0,1)$ & $-\theta / \log (1-\theta)$ \\
\hline
\end{tabular}


em que $p_{0}=P(M=0)$ é a fração de cura, como descrita no modelo de fatores latentes. Além disto, será assumido que os tempos de ativação seguem a distribuição exponencial generalizada (GE) com parâmetros $\lambda>0$ e $\alpha>0$, introduzida por Gupta \& Kundu (1999). Como mencionado em Gupta \& Kundu (1999), a função de taxa de falha desta distribuição é diferente à correspondente da distribuição de Weibull, isto é, para $\alpha>1$ esta função é crescente e vai de 0 até $\lambda$ (em lugar de 0 a $\infty$, como na Weibull), para $\alpha<1$ é decrescente e vai de $\infty$ até $\lambda$ (em lugar de $\infty$ até 0 , como na Weibull), e para $\alpha=1$ esta função é constante (como na Weibull). As funções de distribuição cumulativa, de sobrevivência e de densidade para a distribuição GE são dadas, para tudo $t>0$, por

$$
\begin{aligned}
& F_{a}(t)=\left(1-e^{-\lambda t}\right)^{\alpha}, \\
& S_{a}(t)=1-\left(1-e^{-\lambda t}\right)^{\alpha}
\end{aligned}
$$

$\mathrm{e}$

$$
f_{a}(t)=\alpha \lambda e^{-\lambda t}\left(1-e^{-\lambda t}\right)^{\alpha-1}
$$

respectivamente.

Com estas suposições os modelos com fração de cura derivados com os esquemas da primeira, da última e o aleatório com distribuição uniforme serão denotados por GEPSF, GEPSL e GEPSR, respectivamente. As funções de sobrevivência destes modelos são obtidos de (2.20), (2.45) e (2.61) e são dadas por

$$
\begin{aligned}
& S_{p F}(t)=\frac{C\left(\theta S_{a}(t)\right)}{C(\theta)} \\
& S_{p L}(t)=1+\frac{a_{0}}{C(\theta)}-\frac{C\left(\theta\left(1-S_{a}(t)\right)\right.}{C(\theta)}
\end{aligned}
$$

$\mathrm{e}$

$$
S_{p R}(t)=\frac{a_{0}}{C(\theta)}+\left(1-\frac{a_{0}}{C(\theta)}\right) S_{a}(t)
$$

respectivamente. Note-se que $\theta S_{a}(t)<\theta$ e $\theta\left(1-S_{a}(t)\right)<\theta$, se $0<S_{a}(t)<1$.

Quando é considerada a distribuição de Poisson os modelos com fração de cura derivados com os esquemas da primeira da última e aleatório com distribuição uniforme serão denotados por GEPF, GEPL e GEPR, respectivamente. Similarmente quando é considerada a distribuição geométrica os modelos de fração de cura derivados os esquemas da primeira da última e aleatório com distribuição uniforme serão denotados por GEGF, GEGL and GEGR, respectivamente. Finalmente quando é considerada 
a distribuição logarítmica os modelos com fração de cura com estes esquemas de ativação serão denotados por GELF, GELL and GELR, respectivamente. As funções de sobrevivência destes modelos são obtidas substituindo as correspondentes $C(\theta)$ e $a_{0}$, dados na Tabela 4.1, em (4.5), (4.6) e (4.7), respectivamente. Agora, como feito em Cancho et al. (2013), a fração de cura $p_{0}$ é incorporada nestas funções com a reparametrização $\theta=C^{-1}\left(a_{0} / p_{0}\right)$. Assim, para as distribuições Poisson, geométrica e logarítmica $\theta$ converte-se em $-\log \left(p_{0}\right), 1-p_{0}$ e $1+p_{0} W\left(-p_{0} e^{-1 / p_{0}}\right)$, respectivamente, em que $W(\cdot)$ denota a função de Lambert $W$ (Corless et al., 1996). Com esta reparametrização as funções estendidas de sobrevivência e de densidade destes modelos são dadas na Tabela 4.2, como em Cancho et al. (2013).

Tabela 4.2: Funções de sobrevivência $\left(S_{\mathrm{p}}\right)$ e de densidade $\left(f_{\mathrm{p}}\right)$ para alguns modelos

\begin{tabular}{lcc}
\hline Model & $S_{\mathrm{p}}(t)$ & $f_{\mathrm{p}}(t)$ \\
\hline GEPF & $p_{0}^{F_{a}(t)}$ & $-\log \left(p_{0}\right) p_{0}^{F_{a}(t)} f_{a}(t)$ \\
GEPL & $1+p_{0}-p_{0}^{S_{a}(t)}$ & $-\log \left(p_{0}\right) p_{0}^{S_{a}(t)} f_{a}(t)$ \\
GEPR & $p_{0}+\left(1-p_{0}\right) S_{a}(t)$ & $\left(1-p_{0}\right) f_{a}(t)$ \\
\hline GEGF & $\left\{1+\left(p_{0}^{-1}-1\right) F_{a}(t)\right\}^{-1}$ & $\frac{p_{0}^{-1}-1}{\left\{1+\left(p_{0}^{-1}-1\right) F_{a}(t)\right\}^{2}} f_{a}(t)$ \\
GEGL & $1+p_{0}-\left\{1+\left(p_{0}^{-1}-1\right) S(y)\right\}^{-1}-1$ \\
GEGR & $p_{0}+\left(1-p_{0}\right) S_{a}(t)$ & $\frac{p_{0}^{-1}}{\left\{1+\left(p_{0}^{-1}-1\right) S_{a}(t)\right\}^{2}(t)}$ \\
GELF & $-\frac{\log \left(1-W_{0} S_{a}(t)\right)}{W_{0} S_{a}(t)} p_{0}$ & $\frac{W_{0} S_{a}(t)+\left\{1-W_{0} S_{a}(t)\right\} \log \left(1-W_{0}(t)\right.}{\left\{1-W_{0} S_{a}(t)\right\} W_{0} S_{a}(t)^{2}}$ \\
GELL & $1+p_{0}+\frac{\log \left(1-W_{0} F_{a}(t)\right)}{W_{0} F_{a}(t)} p_{0} f_{a}(t)$ & $\frac{W_{0} F_{a}(t)+\left\{1-W_{0} F_{a}(t)\right\} \log \left(1-W_{0} F_{a}(t)\right)}{\left\{1-W_{0} F_{a}(t)\right\} W_{0} F_{a}(t)^{2}} p_{0} f_{a}(t)$ \\
GELR & $p_{0}+\left(1-p_{0}\right) S_{a}(t)$ & $\left(1-p_{0}\right) f_{a}(t)$ \\
\hline
\end{tabular}

Nota. $W_{0}=1+p_{0} W\left(-e^{-1 / p_{0}} / p_{0}\right)$, em que $W(\cdot)$ é a função de Lambert (Corless et al., 1996).

As funções $f_{p}(t)$ e $S_{p}(t)$ têm três parâmetros, $\alpha, \lambda$ e $p_{0}$. Assim, para tudo $t>0, \alpha>0$ $\lambda>0$ e $p_{0} \in(0,1)$, são definidos

$\operatorname{com} \gamma=(\alpha, \lambda)^{\top}$.

$$
\begin{aligned}
f_{p}\left(t, \gamma, p_{0}\right) & =f_{p}\left(t, \alpha, \lambda, p_{0}\right)=f_{p}(t) \\
S_{p}\left(t, \gamma, p_{0}\right) & =S_{p}\left(t, \alpha, \lambda, p_{0}\right)=S_{p}(t),
\end{aligned}
$$

Como foi mencionado na Seção 1.1, a fração de cura para o $i$-ésimo individuo em uma amostra de tamanho $n$ é dada pela regressão logística entre $p_{0}$ e as co-variáveis como 
na Equação (1.3):

$$
p_{0 i}=\frac{\exp \left(\boldsymbol{x}_{i}^{\top} \boldsymbol{\beta}\right)}{1+\exp \left(\boldsymbol{x}_{i}^{\top} \boldsymbol{\beta}\right)},
$$

em que $\boldsymbol{x}_{i}=\left(x_{i 1}, \ldots, x_{i k}\right)^{\top}$ é o vetor dos valores correspondentes às covariáveis e $\boldsymbol{\beta}=\left(\beta_{1}, \ldots, \beta_{k}\right)^{\top}$ é o vetor de coeficientes das covariáveis. Com esta função de ligação os modelos são identificáveis no sentido de Li et al. (2001). Neste caso os modelos gerados pelo esquema aleatório com distribuição uniforme são iguais ao modelo com fração de cura proposto por Kannan et al. (2010), isto é, GEPR=GEGR=GELR=GE.

\subsection{Inferência}

Considere-se que para o $i$-ésima unidade de uma amostra de tamanho $n$, é observado $t_{i}=\min \left\{T_{i}, C_{i}\right\}$ e $\delta_{i}=\mathrm{I}\left(T_{i} \leq C_{i}\right)$, com $T_{i}$ o tempo até a ocorrência do evento de interesse, $C_{i}$ o tempo de censura e $\mathrm{I}\left(T_{i} \leq C_{i}\right)$ é a função indicadora.

Seja $\boldsymbol{\psi}^{\top}=\left(\boldsymbol{\gamma}^{\top}, \boldsymbol{\beta}^{\top}\right)$ o vetor de parâmetros do modelo e $\Psi \subset \mathbb{R}^{2+} \times \mathbb{R}^{k}$ o espaço paramétrico. A função de verossimilhança associada com $\left(t_{1}, \delta_{1}, \boldsymbol{x}_{1}\right), \ldots,\left(t_{n}, \delta_{n}, \boldsymbol{x}_{n}\right)$ pode ser escrita como

$$
\mathcal{L}(\boldsymbol{\psi} ; \boldsymbol{D})=\prod_{i=1}^{n} f_{p}\left(t_{i}, \boldsymbol{\gamma}, p_{0_{i}}\right)^{\delta_{i}} S_{p}\left(t_{i}, \boldsymbol{\gamma}, p_{0_{i}}\right)^{1-\delta_{i}},
$$

em que $\boldsymbol{D}=(\boldsymbol{t}, \boldsymbol{\delta}, \boldsymbol{X}), \boldsymbol{t}=\left(t_{1}, \ldots, t_{n}\right)^{\top}, \boldsymbol{x}=\left(\boldsymbol{x}_{1}, \ldots, \boldsymbol{x}_{n}\right)^{\top}, \boldsymbol{X}=\left(\boldsymbol{x}_{\mathbf{1}}^{\top}, \ldots, \boldsymbol{x}_{\boldsymbol{n}}^{\top}\right)$, $\boldsymbol{\delta}=\left(\delta_{1}, \ldots, \delta_{n}\right)^{\top}, f_{p}(\cdot, \cdot, \cdot)$ e $S_{p}(\cdot, \cdot, \cdot)$ são a as funções de densidade e de sobrevivência dadas pelas Equações (4.8).

A função de log-verossimilhança associada como $\boldsymbol{D}$ pode ser expressa como

$$
\ell(\boldsymbol{\psi})=\sum_{i=1}^{n}\left[\delta_{i} \log \left(f_{p}\left(t_{i} ; \boldsymbol{\gamma}, p_{0_{i}}\right)\right)+\left(1-\delta_{i}\right) \log \left(S_{p}\left(t_{i} ; \boldsymbol{\gamma}, p_{0_{i}}\right)\right)\right]
$$

As estimativas de máxima verossimilhança podem ser obtidas maximizando diretamente (4.10)) com a função BBoptim do pacote BB do program $\mathrm{R}$ ( $\mathrm{R}$ Development Core Team, 2011). A função $W$ de Lambert na Tabela 4.2 pode-se encontrar no pacote emdbook do programa R. Sob certas condições de regularidade verifica-se que a distribuição assintótica do estimador de máxima verossimilhança, $\widehat{\boldsymbol{\psi}}$, é normal multivariada (veja Lawless (2003a)) com vetor de meias $\boldsymbol{\psi}$ e cuja matriz de covarianza, $\boldsymbol{\Sigma}(\widehat{\boldsymbol{\psi}})$, pode ser estimada por $\widehat{\boldsymbol{\Sigma}}(\widehat{\boldsymbol{\psi}})=-\ddot{\mathrm{L}}^{-1}(\hat{\boldsymbol{\psi}})$, a matriz de informação de Fisher observada, isto é,

$$
\ddot{\mathrm{L}}(\boldsymbol{\psi})=\frac{\partial^{2} \ell(\boldsymbol{\psi})}{\partial \boldsymbol{\psi} \partial \boldsymbol{\psi}^{\top}}
$$




\subsection{Análise de sensibilidade}

Nesta seção é feito um resumo da metodologia para desenvolver uma análise de sensibilidade.

\subsubsection{Influência global}

Para analisar a influência das observações sobre as estimativas dos parâmetros acostuma-se usar a abordagem case-deletion (vide Cook (1977)) que mede o efeito de remover o $i$-ésimo caso amostral.

Seja $\ell_{(i)}$ a função de log-verossimilhança quando é removido o $i$-ésimo caso amostral. Então, por (4.10)

$$
\ell_{(i)}(\boldsymbol{\psi})=\sum_{j \in\{1, \ldots, n\}-i}\left[\delta_{i} \log \left(f_{p}\left(t_{i} ; \boldsymbol{\gamma}, p_{0_{i}}\right)\right)+\left(1-\delta_{i}\right) \log \left(S_{p}\left(t_{i} ; \boldsymbol{\gamma}, p_{0_{i}}\right)\right)\right]
$$

em que o subíndice "(i)" em uma quantidade significa que a quantidade original é calculada removendo o $i$-ésimo caso. Seja $\hat{\boldsymbol{\psi}}_{(i)}=\left(\hat{\gamma}_{(i)}^{\top}, \hat{\boldsymbol{\beta}}_{(i)}^{\top}\right)^{\top}$ a estimativa de máxima verossimilhança de $\ell_{(i)}(\boldsymbol{\psi})$. Assim, o $i$-ésimo caso é considerado como uma observação influente se a diferença entre $\hat{\boldsymbol{\psi}}_{(i)}$ e $\hat{\boldsymbol{\psi}}$ é grande.

Desde que $\hat{\boldsymbol{\psi}}_{(i)}$ deve-se calcular para os $n$ casos, a seguinte aproximação, dado em Cook \& Weisberg (1982), é usada para diminuir o tempo computacional:

$$
\hat{\boldsymbol{\psi}}_{(i)} \approx \hat{\boldsymbol{\psi}}-\ddot{\mathrm{L}}^{-1}(\hat{\boldsymbol{\psi}}) \dot{\ell}_{(i)}(\hat{\boldsymbol{\psi}})
$$

em que $\dot{\ell}_{(i)}(\hat{\boldsymbol{\psi}})$ é a derivada $\frac{\partial \ell_{(i)}(\boldsymbol{\psi})}{\partial \boldsymbol{\psi}}$ avaliada em $\boldsymbol{\psi}=\hat{\boldsymbol{\psi}}$.

Substituindo em (4.13) as descomposições

$\mathrm{e}$

$$
\begin{aligned}
\dot{\ell}_{(i)}(\hat{\boldsymbol{\psi}}) & =\left[\begin{array}{c}
\frac{\partial \ell_{(i)}(\hat{\boldsymbol{\psi}})}{\partial \gamma} \\
\left.\frac{\partial \ell_{(i)}(\hat{\boldsymbol{\psi}})}{\partial \boldsymbol{\beta}}\right)
\end{array}\right] \\
-\ddot{\mathrm{L}}^{-1}(\hat{\boldsymbol{\psi}}) & =\left[\begin{array}{cc}
A_{\boldsymbol{\gamma}} & A_{\boldsymbol{\gamma} \boldsymbol{\beta}} \\
A_{\boldsymbol{\gamma} \boldsymbol{\beta}}^{\top} & A_{\boldsymbol{\beta}}
\end{array}\right]
\end{aligned}
$$

são obtidas as seguintes aproximações:

$$
\begin{aligned}
& \hat{\boldsymbol{\gamma}}_{(i)} \approx \boldsymbol{\gamma}-\left(A_{\boldsymbol{\gamma}} \frac{\partial \ell_{(i)}(\hat{\boldsymbol{\psi}})}{\partial \boldsymbol{\gamma}}+A_{\boldsymbol{\gamma} \boldsymbol{\beta}} \frac{\partial \ell_{(i)}(\hat{\boldsymbol{\psi}})}{\partial \boldsymbol{\beta}}\right) \\
& \hat{\boldsymbol{\beta}}_{(i)} \approx \boldsymbol{\beta}-\left(A_{\boldsymbol{\beta}} \frac{\partial \ell_{(i)}(\hat{\boldsymbol{\psi}})}{\partial \boldsymbol{\beta}}+A_{\boldsymbol{\gamma} \boldsymbol{\beta}}^{\top} \frac{\partial \ell_{(i)}(\hat{\boldsymbol{\psi}})}{\partial \boldsymbol{\gamma}}\right) .
\end{aligned}
$$

Medidas usuais para a diferença entre $\hat{\boldsymbol{\psi}}_{(i)}$ e $\hat{\boldsymbol{\psi}}$ são detalhadas na seguinte seção. 


\subsubsection{A distância generalizada de Cook}

Uma medida para a diferença entre $\hat{\boldsymbol{\psi}}_{(i)}$ e $\hat{\boldsymbol{\psi}}$, denotada $G D_{i}$, é a distância generalizada de Cook

$$
G D_{i}=\left(\hat{\boldsymbol{\psi}}_{(i)}-\hat{\boldsymbol{\psi}}\right)^{\top}(-\ddot{\mathrm{L}}(\hat{\boldsymbol{\psi}}))\left(\hat{\boldsymbol{\psi}}_{(i)}-\hat{\boldsymbol{\psi}}\right)
$$

Desde que $\hat{\boldsymbol{\psi}}^{\top}$ maximiza a função de log-verossimilhança, - ̈L é definida positiva e $G D_{i}$ é, com efeito, uma distância matemática. Assim, a distância entre $\hat{\boldsymbol{\psi}}_{(i)}$ e $\hat{\boldsymbol{\psi}}$ é uma medida da influência do $i$-ésimo caso amostral sobre o vetor de estimativas dos parâmetros. Da Equação (4.13) obtém-se a seguinte aproximação para obter esta distância

$$
G D_{i} \approx \dot{\ell}_{(i)}(\hat{\boldsymbol{\psi}})^{\top}\left(-\ddot{\mathrm{L}}^{-1}(\hat{\boldsymbol{\psi}})\right) \dot{\ell}_{(i)}(\hat{\boldsymbol{\psi}})
$$

Agora algumas medidas de influência do $i$-ésimo caso amostral sobre os subvetores de estimativas dos parâmetros, $\hat{\boldsymbol{\gamma}}$ e $\hat{\boldsymbol{\beta}}$, são derivadas seguindo a Li et al. (2012). Substituindo (4.16) e (4.17) em (4.19) segue que

$$
G D_{i} \approx \frac{\partial \ell_{(i)}(\hat{\boldsymbol{\psi}})^{\top}}{\partial \boldsymbol{\gamma}} A_{\boldsymbol{\gamma}} \frac{\partial \ell_{(i)}(\hat{\boldsymbol{\psi}})}{\partial \boldsymbol{\gamma}}+2 \frac{\partial \ell_{(i)}(\hat{\boldsymbol{\psi}})^{\top}}{\partial \boldsymbol{\gamma}} A_{\boldsymbol{\gamma} \boldsymbol{\beta}} \frac{\partial \ell_{(i)}(\hat{\boldsymbol{\psi}})}{\partial \boldsymbol{\beta}}+\frac{\partial \ell_{(i)}(\hat{\boldsymbol{\psi}})^{\top}}{\partial \boldsymbol{\beta}} A_{\boldsymbol{\beta}} \frac{\partial \ell_{(i)}(\hat{\boldsymbol{\psi}})}{\partial \boldsymbol{\beta}} .
$$

Desta aproximação, as seguintes quantidades

$\mathrm{e}$

$$
G D_{i}(\gamma) \approx \frac{\partial \ell_{(i)}(\hat{\boldsymbol{\psi}})^{\top}}{\partial \gamma} A_{\gamma} \frac{\partial \ell_{(i)}(\hat{\boldsymbol{\psi}})}{\partial \gamma}
$$

$$
G D_{i}(\boldsymbol{\beta}) \approx \frac{\partial \ell_{(i)}(\hat{\boldsymbol{\psi}})^{\top}}{\partial \boldsymbol{\beta}} A_{\boldsymbol{\beta}} \frac{\partial \ell_{(i)}(\hat{\boldsymbol{\psi}})}{\partial \boldsymbol{\beta}}
$$

são consideradas medidas da influência do $i$-ésimo caso amostral sobre as estimativas $\hat{\boldsymbol{\gamma}}$ e $\hat{\boldsymbol{\beta}}$, respectivamente.

\subsubsection{O desvio da verossimilhança}

O desvio da log-verosimilhanca de $\hat{\boldsymbol{\psi}}_{(i)}$ com respeito a $\hat{\boldsymbol{\psi}}$ é dado por

$$
L D_{i}=2\left\{\ell(\hat{\boldsymbol{\psi}})-\ell\left(\hat{\boldsymbol{\psi}}_{(i)}\right)\right\}
$$

Porquanto $\hat{\boldsymbol{\psi}}^{\top}$ maximiza a função de log-verossimilhança $L D_{i}$ é não negativa, mas não uma distância matemática. Como é salientado em Cook (1986), esta medida é amplamente usada por suas propriedades assintóticas, como por exemplo que

$$
\left\{\boldsymbol{\psi}: 2[\ell(\hat{\boldsymbol{\psi}})-\ell(\boldsymbol{\psi})]<\chi^{2}\right\}
$$


é uma região de confidência para $\boldsymbol{\psi}$, com $\chi^{2}$ um quantile da distribuição chi-quadrado com $k+2$ graus de liberdade. Desde que

$$
L D(\boldsymbol{\psi}) \approx(\boldsymbol{\psi}-\hat{\boldsymbol{\psi}})^{\top}(-\ddot{\mathrm{L}}(\hat{\boldsymbol{\psi}}))(\boldsymbol{\psi}-\hat{\boldsymbol{\psi}}),
$$

em uma vizinhança $\hat{\boldsymbol{\psi}}$

$$
L D_{i} \approx G D_{i},
$$

sempre que $\hat{\boldsymbol{\psi}}_{(i)}$ esteja próximo de $\hat{\boldsymbol{\psi}}$, como foi observado em Li et al. (2012).

\subsubsection{Influência local}

Uma abordagem diferente para desenvolver uma análise de sensibilidade considera o efeito de pequenas perturbações no modelo ou nos dados, segundo um esquema particular. Esta abordagem, chamada influência local, foi proposta por Cook (1986) para o modelo de regressão e depois foi estendida para modelos mais gerais, como é descrito em Escobar \& Meeker (1992). Seja $\boldsymbol{w}=\left(w_{1}, \ldots, w_{n}\right)$ um vetor de números que quantificam perturbação e $\ell W(\boldsymbol{\psi}, \boldsymbol{w})$ a corresponde função de log-verossimilhança, com $\boldsymbol{\psi} \in \Psi$ e $\boldsymbol{w} \in \Omega$ (um subconjunto de $\mathbb{R}^{n}$ ). É assumido que existe $\boldsymbol{w}_{0}$ tal que quando $\boldsymbol{w}=\boldsymbol{w}_{0}$ não ocorre a perturbação. Assim, $\ell W\left(\boldsymbol{\psi}, \boldsymbol{w}_{0}\right)=\ell(\boldsymbol{\psi})$ e $\hat{\boldsymbol{\psi}}$ maximiza $\ell W\left(\cdot, \boldsymbol{w}_{0}\right)$.

Com esta abordagem, o desvio da função de log-verossimilhança (Cook \& Weisberg, 1982), $L D W$, é definido por

$$
L D W(\boldsymbol{w})=2\left\{\ell(\hat{\boldsymbol{\psi}})-\ell\left(\hat{\boldsymbol{\psi}}_{w}\right)\right\},
$$

em que $\hat{\boldsymbol{\psi}}_{w}$ maximiza $\ell W(\cdot, \boldsymbol{w})$. Como é mencionado em Beckman et al. (1987), grandes valores de $L D W$ indicam que $\hat{\boldsymbol{\psi}}$ e $\hat{\boldsymbol{\psi}}_{W}$ diferem consideravelmente en relação com as curvas de nível da função log-verossimilhança sem perturbar, $\ell(\boldsymbol{\psi})$. Cook (1986) estabelece que o gráfico da superfície $(\boldsymbol{w}, L D W(\boldsymbol{w}))$ contem informação essencial sobre a influência do esquema de perturbação usado e para medir esta influência usa curvaturas como se describe a seguir.

A curvatura de $L D W$, na direção do vetor unitário $\boldsymbol{h}$, avaliada en $\boldsymbol{w}_{0}$ é dada por

com

$$
C_{\boldsymbol{h}}=\frac{\partial^{2} L D\left(\boldsymbol{w}_{0}+a h\right)}{\partial a^{2}}=2 \boldsymbol{h}^{\top} \ddot{A} \boldsymbol{h},
$$

$$
\ddot{A}=-\frac{\partial^{2} L\left(\hat{\boldsymbol{\psi}}_{w}, \boldsymbol{w}_{0}\right)}{\partial \boldsymbol{w} \partial \boldsymbol{w}^{\top}} .
$$

De (4.25) a máxima curvatura, $C_{\text {max }}$, é obtida quando $\boldsymbol{h}$ é o autovetor, $\boldsymbol{h}_{\max }$, associado com o maior autovalor de $\ddot{A}$. Assim, $\boldsymbol{h}_{\max }=\left(\boldsymbol{h}_{\max _{1}}, \ldots, \boldsymbol{h}_{\max _{n}}\right)^{\top}$ indica como perturbar 
o modelo ou os dados para obter o maior cambio local em $L D W$. De acordo com Cook (1986), o gráfico dos pontos $\left(i, \boldsymbol{h}_{\text {max }_{i}}\right), i=1, \ldots, n$, identifica casos potencialmente influentes, além disto $\boldsymbol{h}_{\text {max }_{i}}>2$ pode ser usado como marca de referência para indicar que a $i$-ésima observação é influente.

Outras marcas de referência que servem para indicar se uma observação é influente são dadas a seguir. Sejam $\boldsymbol{u}_{i} \in \mathbb{R}^{n}$ o vetor de zeros com um 1 na $i$-ésima posição, $C_{i}=2 A_{i i}$ a curvatura associada com $\boldsymbol{u}_{i}$ e $M(0)_{i}=C_{i} / \sum_{j=1}^{k+2} \lambda_{j}, i=1, \ldots, n$, em que $\lambda_{1} \geq \cdots \geq \lambda_{k+2}$ são os $k+2$ autovalores positivos de $2 \ddot{A}$. Agora, sejam $\bar{M}(0)$ e $S_{M(0)}$ a média e a desvio padrão, respectivamente, dos valores $M(0)_{1}, \ldots, M(0)_{n}$. Note que $\bar{M}(0)=1 / n$, porque $\sum_{j=1}^{k+2} \lambda_{j}=\operatorname{trace}(2 \ddot{A})$. O desenho dos pontos $\left(i, M(0)_{i}\right), i=1, \ldots, n$, é outra maneira gráfica para identificar casos influentes. Poon \& Poon (1999), Zhu \& Lee (2001) e Escobar \& Meeker (1992) propuseram as marcas de referências $M(0)_{i}>2 / n, M(0)_{i}>1 / n+2 S_{M(0)}$ e $M(0)_{i}>\chi_{0,5 ; k+2}^{2} / \sum_{j=1}^{k+2} \lambda_{j}$, respectivamente, para indicar que a $i$-ésima observação pode ser considerada influente.

Assim, a matriz $\ddot{A}$ é chave para levar a cabo a análise de influência local. A identidade seguinte, dada em Cook (1986), é usada para facilitar o cálculo de $\ddot{A}$ :

com

$$
\ddot{A}=\Delta^{\top}(-\ddot{L}(\hat{\psi}))^{-1} \Delta,
$$

$$
\Delta=\frac{\partial^{2} \ell W\left(\hat{\boldsymbol{\psi}}, \boldsymbol{w}_{0}\right)}{\partial \boldsymbol{\psi} \partial \boldsymbol{w}^{\top}}
$$

Conseqüentemente é importante calcular a matriz $\Delta$.

A seguir são descritos os esquemas de perturbação, que serão usados neste trabalho, e serão derivadas algumas identidades para obter a matiz $\Delta$.

\subsubsection{Perturbação da verossimilhança}

Neste caso, o vetor $\boldsymbol{w}=\left(w_{1}, \ldots, w_{n}\right)$, usado para quantificar a perturbação, afeta à função log-verossimilhança como segue

$$
\ell W(\boldsymbol{\psi}, \boldsymbol{w})=\sum_{l=1}^{n}\left[w_{l} \delta_{l} \log \left(f_{p}\left(t_{l}, \boldsymbol{\gamma}, p_{0_{l}}\right)\right)+w_{l}\left(1-\delta_{l}\right) \log \left(S_{p}\left(t_{l}, \boldsymbol{\gamma}, p_{0_{l}}\right)\right)\right],
$$

com $0 \leq w_{i} \leq 1$. O vetor de não-perturbação é $\boldsymbol{w}_{0}=(1, \ldots, 1)^{\top}$.

Desde que $\frac{\partial^{2} \ell W(\boldsymbol{\psi}, \boldsymbol{w})}{\partial \psi_{j} \partial w_{i}}=\frac{\partial}{\partial \psi_{j}} G W_{i}(\boldsymbol{\psi})$, com

$$
G W_{i}(\boldsymbol{\psi})=\delta_{i} \log \left(f_{p}\left(t_{i}, \boldsymbol{\gamma}, p_{0_{i}}\right)\right)+\left(1-\delta_{i}\right) \log \left(S_{p}\left(t_{i}, \boldsymbol{\gamma}, p_{0_{i}}\right)\right),
$$


O cálculo computacional de $\Delta$ é direto da matriz hessiana de $G W(\boldsymbol{\psi})=$ $\left(G W_{1}(\boldsymbol{\psi}), \ldots, G W_{n}(\boldsymbol{\psi})\right)$, que deve-se avaliar em $\hat{\boldsymbol{\psi}}$.

\subsubsection{Perturbação da resposta}

Nesta caso a perturbação é originada nas respostas, $t i$, a partir de $t_{i}+w_{i} s_{T}, i=1, \ldots, n$, $\operatorname{com} \boldsymbol{w}=\left(w_{1}, \ldots, w_{n}\right)$ o vetor usado para quantificar a perturbação e $s_{T}$ é um fator de escala estimado pelo desvio padrão das respostas. O vetor de não-perturbação é $\boldsymbol{w}_{0}=(0, \ldots, 0)^{\top}$.

A função de log-verossimilhança perturbada é dada por

$$
\ell W(\boldsymbol{\psi}, \boldsymbol{w})=\sum_{l=1}^{n}\left[\delta_{l} \log \left(f_{p}\left(t_{l}+w_{l} s_{t}, \boldsymbol{\gamma}, p_{0_{l}}\right)\right)+\left(1-\delta_{l}\right) \log \left(S_{p}\left(t_{l}+w_{l} s_{t}, \boldsymbol{\gamma}, p_{0_{l}}\right)\right)\right]
$$

Desta equação segue que $\frac{\partial}{\partial w_{i}} \ell W\left(\boldsymbol{\psi}, \boldsymbol{w}_{0}\right)=G R_{i}(\boldsymbol{\psi})$, com

$$
G R_{i}(\boldsymbol{\psi})=s_{T}\left\{\frac{\delta_{i} \frac{\partial}{\partial t} f_{p}\left(t_{i}+s_{T}, \boldsymbol{\gamma}, p_{0_{i}}\right)}{f_{p}\left(t_{i}+s_{T}, \boldsymbol{\gamma}, p_{0_{i}}\right)}-\frac{\left(1-\delta_{i}\right) f_{p}\left(t_{i}+s_{T}, \boldsymbol{\gamma}, p_{0_{i}}\right)}{S_{p}\left(t_{i}+s_{T} ; \boldsymbol{\gamma}, p_{0_{i}}\right)}\right\} .
$$

O cálculo computacional de $\Delta$ é direto da matriz hessiana de $G R(\boldsymbol{\psi})=$ $\left(G R_{1}(\boldsymbol{\psi}), \ldots, G R_{n}(\boldsymbol{\psi})\right)$, que deve-se avaliar em $\hat{\boldsymbol{\psi}}$.

\subsubsection{Perturbação das covariáveis}

Nesta caso, a perturbacao é originada en uma das cocoriáveis. Se $\left(x_{1 k_{o}}, \ldots, x_{n k_{o}}\right)$ é o vetor de valores de $\boldsymbol{X}_{k_{o}}$ na amostra, então o vetor de valores perturbados é $\left(x_{1 k_{o}}+w_{1} s_{\boldsymbol{X}_{k o}}, \ldots, x_{n k_{o}}+w_{n} s_{\boldsymbol{X}_{k o}}\right)$, em que $s_{\boldsymbol{X}_{k o}}$ é o desvio padrão dos valores observados desta covariável. O vetor de não-perturbação é $\boldsymbol{w}_{0}=(0, \ldots, 0)^{\top}$. Seja $\boldsymbol{x} \boldsymbol{w}_{i}$ o vetor obtido quando a $k_{o}$-ésimo componente em $\boldsymbol{x}_{i}=\left(x_{i 1}, \ldots, x_{i k}\right)^{\top}$ é substituído por $x_{i k_{o}}+w_{i} s_{\boldsymbol{X}_{k o}}$ e $p w_{0 i}=\frac{\exp \left(\boldsymbol{x} \boldsymbol{w}_{i}^{\top} \boldsymbol{\beta}\right)}{1+\exp \left(\boldsymbol{x} \boldsymbol{w}_{i}^{\top} \boldsymbol{\beta}\right)}$, para $i=1, \ldots, n$. Assim, a função de logverossimilhança perturbada é dada por

$$
\ell W(\boldsymbol{\psi}, \boldsymbol{w})=\sum_{l=1}^{n}\left[\delta_{l} \log \left(f_{p}\left(t_{l}, \boldsymbol{\gamma}, p w_{0 l}\right)\right)+\left(1-\delta_{l}\right) \log \left(S_{p}\left(t_{l}, \boldsymbol{\gamma}, p w_{0 l}\right)\right)\right] .
$$

Desde que

segue que

$$
p w_{0 i}=\frac{\exp \left(\boldsymbol{x}_{i}^{\top} \boldsymbol{\beta}\right) \exp \left(w_{i} s_{\boldsymbol{X}_{k o}} \beta_{k o}\right)}{1+\exp \left(\boldsymbol{x}_{i}^{\top} \boldsymbol{\beta}\right) \exp \left(w_{i} s_{\boldsymbol{X}_{k o}} \beta_{k o}\right)}
$$

$$
\frac{\partial}{\partial w_{i}} \ell W(\boldsymbol{\psi}, \boldsymbol{w})=\frac{\partial}{\partial w_{i}}\left\{\delta_{i} \log \left(f_{p}\left(t_{i}, \boldsymbol{\gamma}, p w_{0 i}\right)\right)+\left(1-\delta_{i}\right) \log \left(S_{p}\left(t_{i}, \boldsymbol{\gamma}, p w_{0 i}\right)\right)\right\}
$$


e, pela regra de cadeia,

$$
\frac{\partial}{\partial w_{i}} \ell W\left(\boldsymbol{\psi}, \boldsymbol{w}_{0}\right)=G E_{i}(\boldsymbol{\psi})
$$

em que

$$
G E_{i}(\boldsymbol{\psi})=\frac{s_{\boldsymbol{X}_{k o}} \beta_{k o} \exp \left(-\boldsymbol{x}_{i}^{\top} \boldsymbol{\beta}\right)}{\left(1+\exp \left(-\boldsymbol{x}_{i}^{\top} \boldsymbol{\beta}\right)\right)^{2}}\left\{\frac{\delta_{i} \frac{\partial}{\partial p} f_{p}\left(t_{i}, \boldsymbol{\gamma}, p_{0_{i}}\right)}{f_{p}\left(t_{i}, \boldsymbol{\gamma}, p_{0_{i}}\right)}+\frac{\left(1-\delta_{i}\right) \frac{\partial}{\partial p} S_{p}\left(t_{i}, \boldsymbol{\gamma}, p_{0_{i}}\right)}{S_{p}\left(t_{i}, \boldsymbol{\gamma}, p_{0_{i}}\right)}\right\} .
$$

O cálculo computacional de $\Delta$ é direto da matriz hessiana de $G E(\boldsymbol{\psi})=$ $\left(G E_{1}(\boldsymbol{\psi}), \ldots, G E_{n}(\boldsymbol{\psi})\right)$, que deve-se avaliar em $\hat{\boldsymbol{\psi}}$.

\subsection{Aplicação}

Serão analisados o conjunto de dados também estudados por Cancho et al. (2013). Estes dados inclui 205 pacientes observados depois de cirurgia para remover um melanoma maligno no período 1962-77. Os dados estão disponíveis no pacote timereg do $\mathrm{R}$ (Scheike, 2009). O tempo observado (T) está entre 0.0274 e 15.25 anos e corresponde ao tempo até a morte do paciente ou o tempo de censura. Os pacientes mortos por outras causas conjuntamente com os pacientes que sobreviveram ao período de estudo são observações censuradas $(72 \%)$. As covariáveis consideradas são $\boldsymbol{X}_{2}=$ ulceration status (presente $=1, n=90$; ausente $=0, n=115), \boldsymbol{X}_{3}=$ tumor thickness (em mm, média $=2.92$ e desvio padrão $=2.96)$ e, a diferencia de Cancho et al. (2013), também são consideradas $\boldsymbol{X}_{4}=$ idade (em anos, média $=52,46$ e desvio padrão $=16,67$ ) e $\boldsymbol{X}_{5}=$ sexo (masculino=1, feminino=0), com coeficientes $\beta_{2}, \beta_{3}, \beta_{4}$ e $\beta_{5}$, respectivamente; e $\beta_{1}$ denota o intercepto. A função de sobrevivência estimada de Kaplan-Meir (veja Figura 4.1) níveis acima de 0.6 e a presença do patamar indicam que um modelo que não considere a possibilidade de cura não deveria ajustar bem estes dados. Assim, os dados podem ser ajustado por um modelos com fração de cura com covariáveis, em particular, os modelos com fração de cura GE-PS. Por tanto, nesta aplicação serão usados os modelos GEPF, GEPL, GEGF, GEGL, GELF, GELL e GE, dados na Tabela 4.2 .

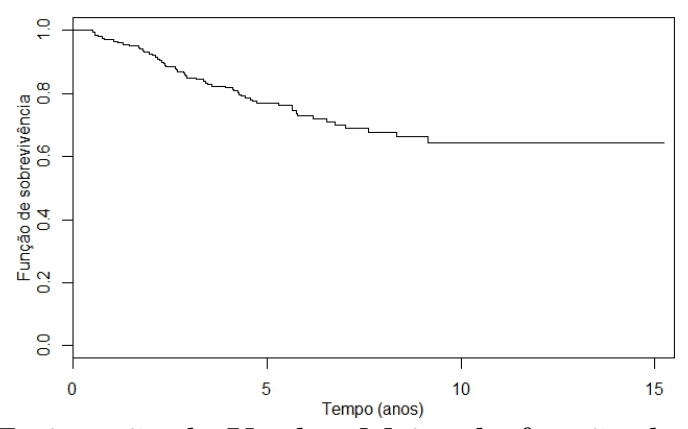

Figura 4.1: Estimação de Kaplan-Meier da função de sobrevivência. 


\subsubsection{Ajuste de modelos e estimativas dos parâmetros}

As estimativas de máxima verossimilhança para os parâmetros, $\boldsymbol{\psi}=\left(\alpha, \lambda, \beta_{1}, \beta_{2}, \ldots, \beta_{5}\right)$, são obtidas maximizando diretamente (4.9) ou (4.10) usando a função BBoptim do programa R (R Development Core Team, 2011). A Tabela 4.3 apresenta os valores máximos da função de log-verossimilhança, $(l(\cdot))$, e os indicadores de ajuste AIC e BIC para as distribuições consideradas.

Tabela 4.3: $A I C$ e $S B C$ para os modelos ajustados.

\begin{tabular}{lccc}
\hline Model & $l(\cdot)$ & $A I C$ & $B I C$ \\
\hline GEPF & $-205,3233$ & 424,6466 & 421,29262 \\
GEGF & $-203,0158$ & 420,0316 & 416,6776 \\
GELF & $-200,9897$ & 415.9794 & 412,6254 \\
GEPL & $-212,0622$ & 438,1244 & 434,77042 \\
GEGL & $-214,9450$ & 443,8900 & 440,53602 \\
GELL & $-217,0028$ & 448,0056 & 444,65162 \\
GE & $-208,1857$ & 430,3714 & 427,01742 \\
\hline
\end{tabular}

Segundo os critérios do $A I C$ e $B I C$, o modelo $G E L F$ é que melhor ajusta aos dados. A Tabela 4.4 mostra as estimativas de máxima verossimilhança (MLE), estimativas dos erros padrões de estimação (SEE) obtidas a partir da matriz de informação de Fisher observada e intervalos de confiança do $95 \%$ de nível para os parâmetros das distribuições ajustadas. Todas as covariáveis, exceto a idade, têm efeito significativo sobre a determinação da fração de cura.

Tabela 4.4: Resumo das estimativas dos parâmetros do modelo GELF.

\begin{tabular}{cccc}
\hline Parâmetro & Estimativa (est) & Erro padrão (see) & Intervalo de confiança \\
\hline$\alpha$ & 2,9685 & 0,6079 & $(1,7771 ; 4,1598)$ \\
$\lambda$ & 0,2227 & 0,0846 & $(0,0569 ; 0,3885)$ \\
$\beta_{1}$ & 1,8318 & 0,625 & $(0,6069 ; 3,0567)$ \\
$\beta_{2}$ & $-1,3875$ & 0,3147 & $(-2,0043 ;-0,7707)$ \\
$\beta_{3}$ & $-0,1141$ & 0,0343 & $(-0,1814 ;-0,0469)$ \\
$\beta_{4}$ & $-0,0041$ & 0,0077 & $(-0,0192 ; 0,011)$ \\
$\beta_{5}$ & $-0,6083$ & 0,2735 & $(-1,1443 ;-0,0723)$ \\
\hline
\end{tabular}




\subsubsection{Análise de resíduos}

Para estudar possíveis desvios do modelo GELF, nesta subseção são considerados os resíduos quantis normalizados aleatorizados, propostos por Dunn \& Smyth (1996). Estes resíduos, denotados por $\hat{r}_{i}$, são obtidos de acordo com Rigby \& Stasinopoulos (2005). Assim, se $t_{i}$ é uma observação não censurada, seu quantil aleatorizado normalizado está definido por ,

$$
\hat{r}_{i}=\Phi^{-1}\left(F_{p}\left(t_{i}, \hat{\alpha}, \hat{\lambda}, \hat{p}_{0_{i}}\right)\right)
$$

em que $\Phi()$ é a função de distribuição cumulativa da distribuição normal padrão. Mas, se $t_{i}$ é uma observação censurada, seu quantil aleatorizado normalizado está definido por

$$
\hat{r}_{i}=\Phi^{-1}\left(u_{i}\right),
$$

em que $u_{i}$ é um valor aleatório da distribuição uniforme no intervalo $\left[F_{p}\left(t_{i}, \hat{\alpha}, \hat{\lambda}, \hat{p}_{0_{i}}\right) ; 1\right]$. Com estes residuais e os quantis correspondentes a distribuição normal padrão é obtido o gráfico Q-Q mostrado no quadro direito da Figura 4.2 conjuntamente com un envelope. Estes gráficos indicam possíveis valores extremos que não são muito bem ajustados pelo modelo. No quadro esquerdo da Figura 4.2 é mostrado o comportamento aleatório dos residuais no intervalo $(-3 ; 3)$.
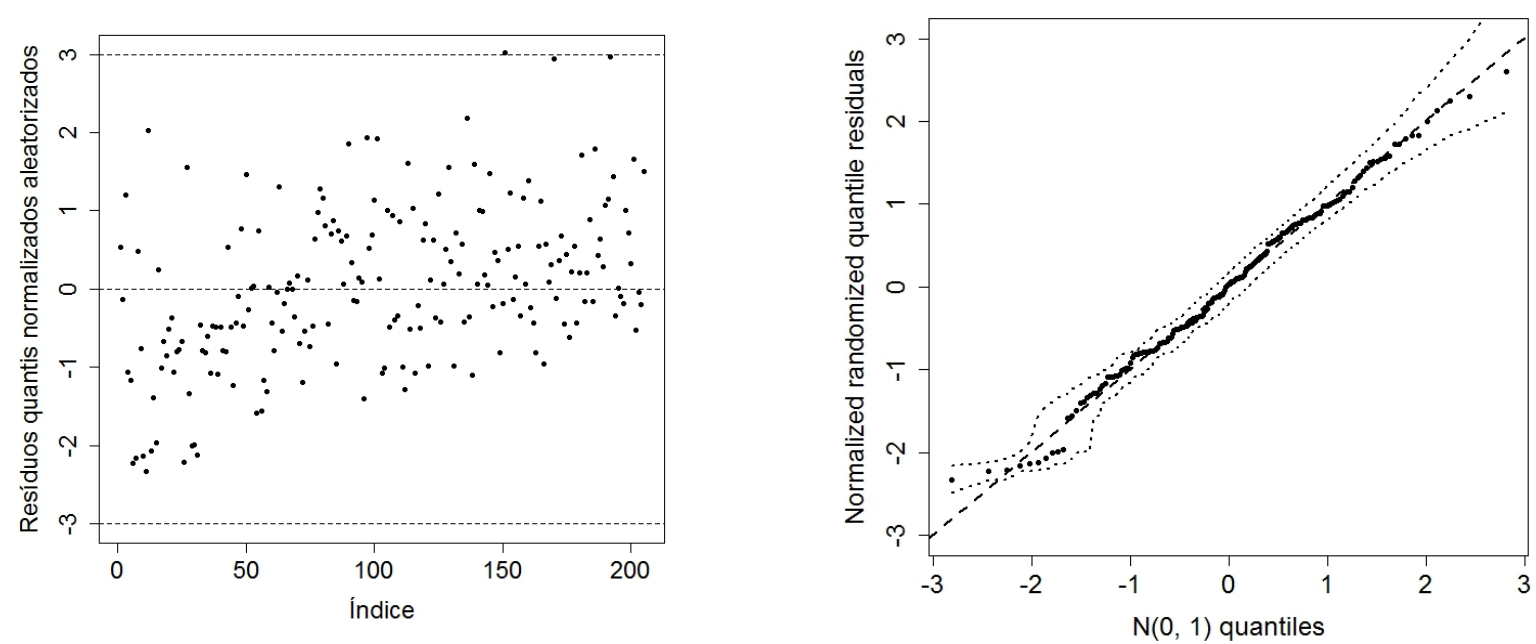

Figura 4.2: Gráficas de resíduos. Quadro esquerdo: Gráfico de índices dos quantis normalizados aleatorizados do modelo ajustado. Quadro direito: Gráfico QQ dos quantis normalizados aleatorizados e envelope gerado. 


\subsubsection{Análise de sensibilidade}

Nesta subseção é feito uma análise de sensibilidade, como descrito na Seção 4.3, para o modelo GELF. São calculadas medidas de influência global e local.

\subsubsection{Influência global}

Para medir a influência do $i$-ésimo caso amostral sobre o vetor de estimativas dos parâmetros, são calculadas as medidas case-deletion $G D_{i}$ e $L D_{i}$ descritas na subsecção 4.3.1. Os valores destas medidas são similares, como é mostrado na Figura 4.3 , isto sugere, segundo (4.23), que remover o $i$-ésimo caso amostral no afetaria significativamente as estimativas dos parâmetros. Observe também da figura 4.3 que os casos 5, 6, 7, 10,11, 13, 15 e 29 estão acima da marca de referência, portanto estes são os mais influentes.
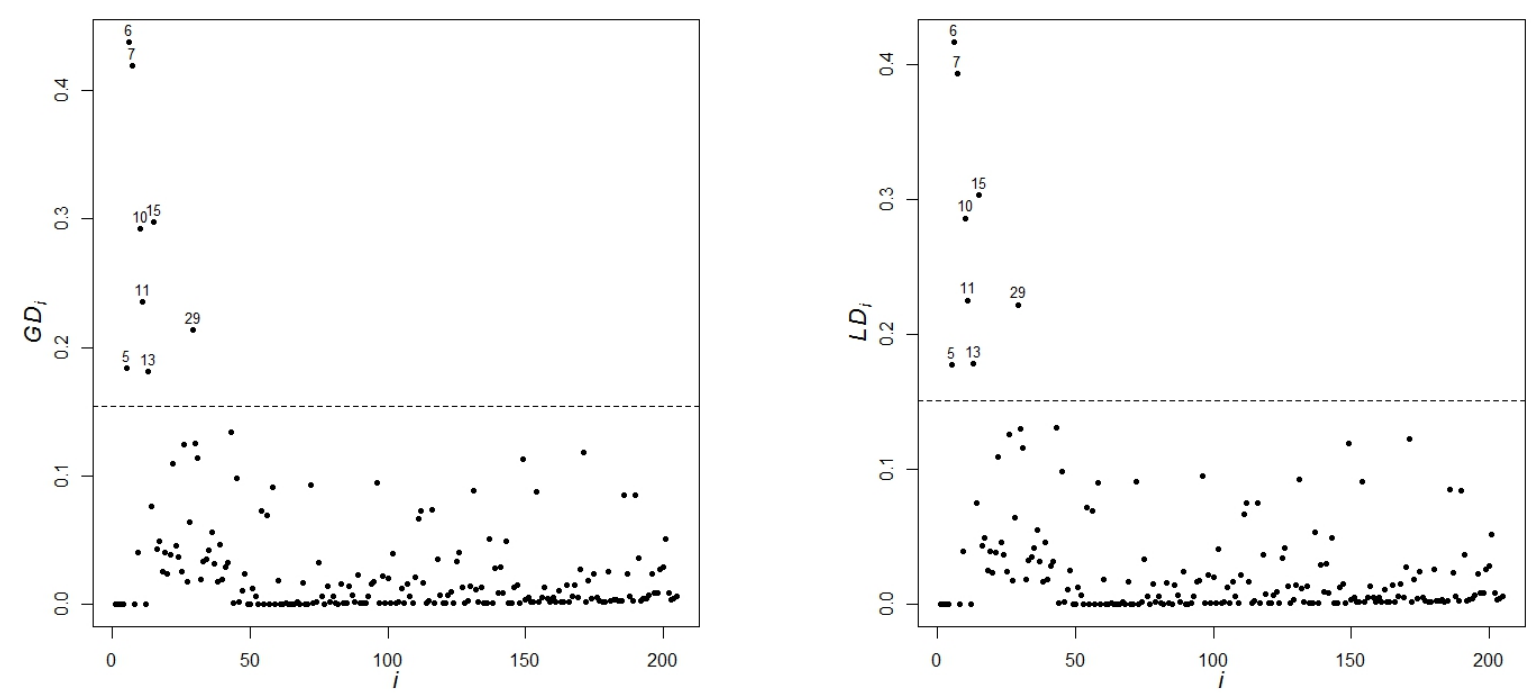

Figura 4.3: Gráfico de índices das medidas case-deletion. Quadro esquerdo: A distância generalizada de Cook $\left(G C_{i}\right)$. Quadro direito: A distância da verossimilhança $\left(L D_{i}\right)$.

Também obtiveram-se medidas de influência sobre os sub-vetores $\boldsymbol{\gamma}=(\alpha, \lambda)$ e $\boldsymbol{\beta}$, os resultados sao ilustrados são mostrados na Figura 4.4. Os casos mais influentes seriam aqueles cujas medidas aparecem por acima da marca de referencia, isto e, os casos 5, $6,7,10,11,26,29$ e 31. 

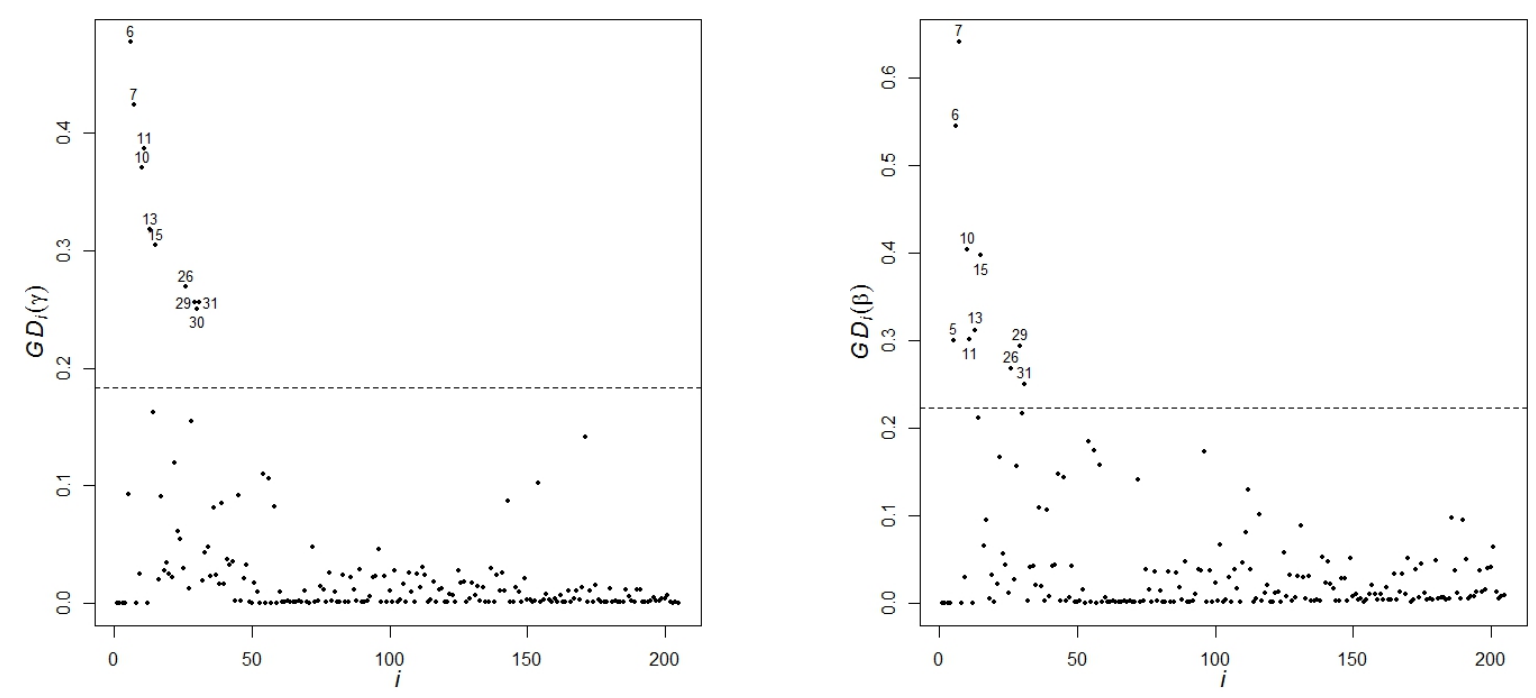

Figura 4.4: Gráfico de índices das medidas case-deletion para os sub-vetores de parâmetros. Quadro esquerdo: A distância generalizada de Cook $G C_{i}(\gamma)$. Quadro direito: A distância generalizada de Cook $G C_{i}(\boldsymbol{\beta})$.

\subsubsection{Influência local}

Nesta sub-subseção são calculadas as medidas de influência descritas na subseção 4.3.2, de acordo com os esquemas de perturbação descritos na subseção 4.3.2, e são feitos os gráficos $\left(i, \boldsymbol{h}_{\text {max }_{i}}\right)$ e $\left(i, M(0)_{i}\right), i=1, \ldots, n$. A matriz $\Delta$ de (4.27) foi obtida com a função hessian do pacote numDeriv do programa $\mathrm{R}$.

Perturbação ponderada de casos. O valor da máxima curvatura é $C_{\max }=2,8160$, que não sugere influência importante. Para uma análise melhor dos possíveis casos influentes são feitos os gráficos mostrados na Figura 4.5. Os casos 5, 6, 7, 11, 13, 15, 29, 30, 43, 72, 111 e 149 são os mais influentes. 

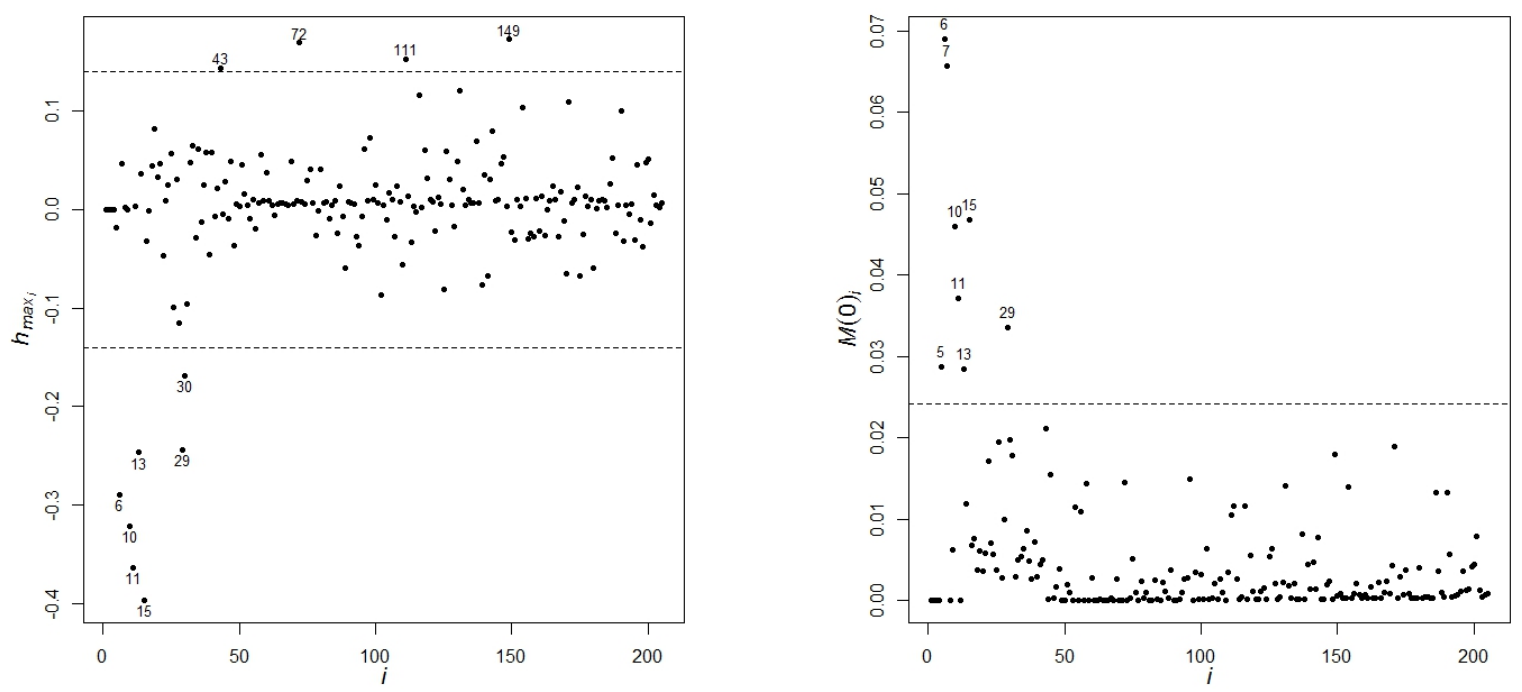

Figura 4.5: Gráficos de índices das medidas de influência segundo o esquema de perturbação ponderada de casos. Quadro esquerdo: Direção da máxima curvatura, $\boldsymbol{h}_{\max }$. Quadro direito: $M(0)_{i}$.

Perturbação da resposta. $O$ valor da máxima curvatura é $C_{\max }=54,7037$. Os casos 5, 6, 7, 9 e 10 seriam os casos mais influentes quando é perturbada a resposta.
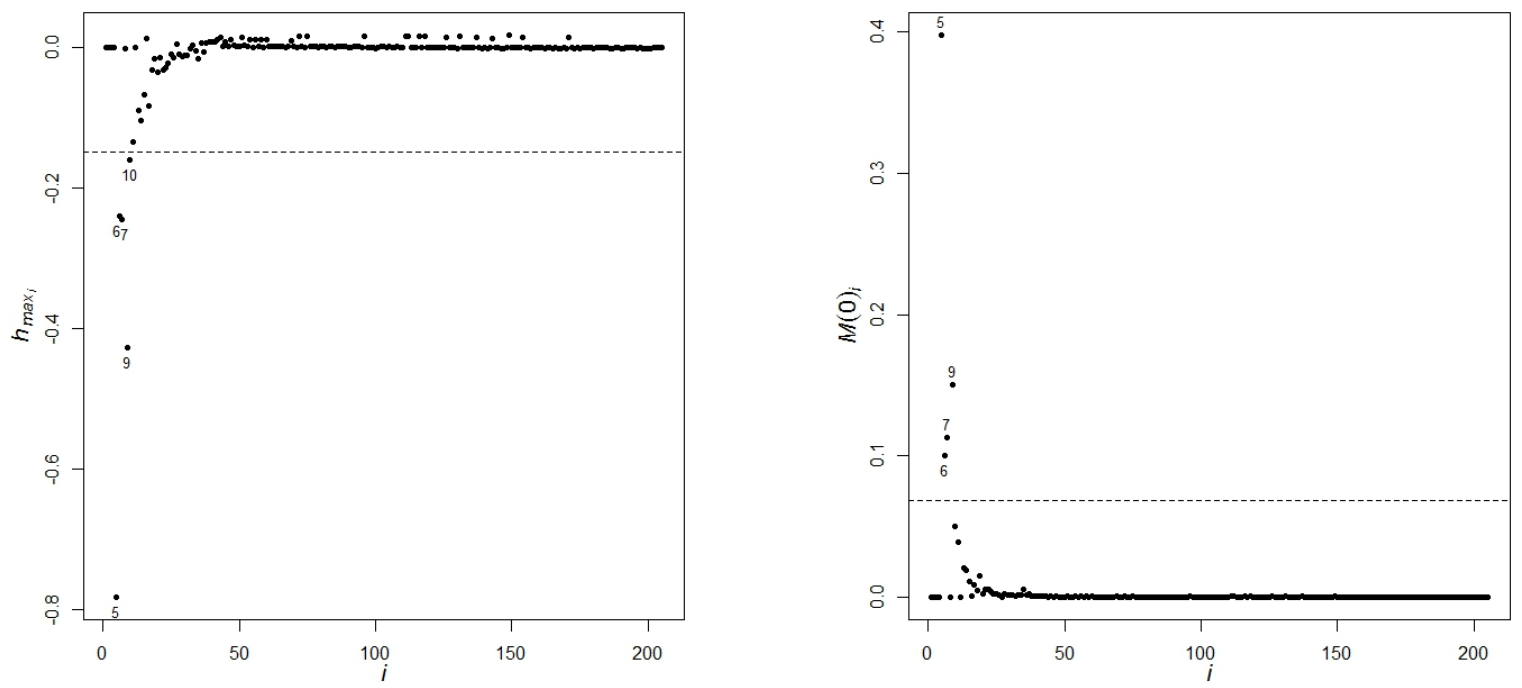

Figura 4.6: Gráficos de índices das medidas de influência segundo o esquema de perturbação ponderada nas respostas. Quadro esquerdo: Direção da máxima curvatura, $\boldsymbol{h}_{\max }$. Quadro direito: $M(0)_{i}$.

Perturbação da covariável espessura. O valor de $C_{\max }=81,7252$ é máxima curvatura. Os gráficos na Figura 4.7 sugerem que os casos 5, 6, 7, 9, 10, 19, 21 e 35 são os mais influentes. 

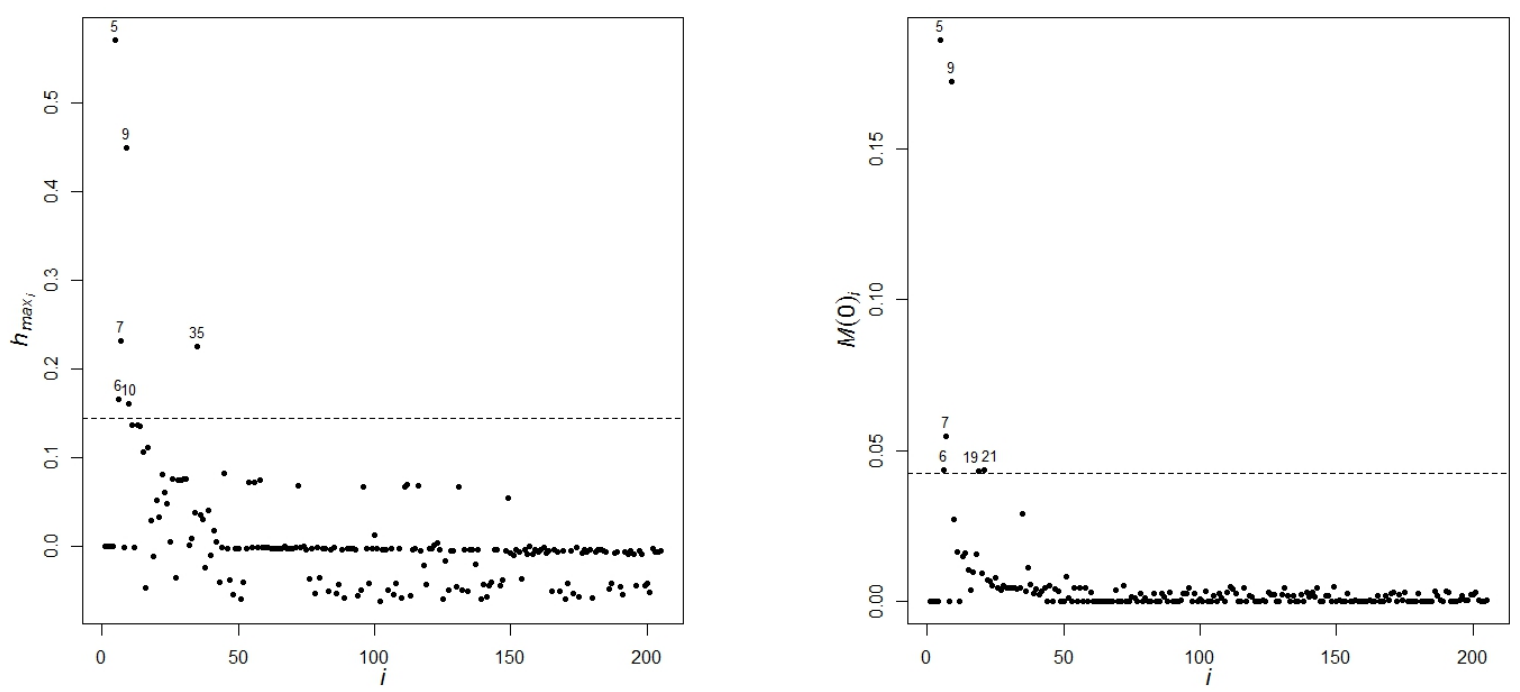

Figura 4.7: Gráficos de índices das medidas de influência local segundo o esquema de perturbação para a co-variável grossura.Quadro esquerdo: Direção da máxima curvatura, $\boldsymbol{h}_{\max }$. Quadro direito: $M(0)_{i}$.

Perturbação da covariável idade. Nesta caso resulta um valor pequeno para a máxima curvatura $C_{\max }=1,0305$. Os casos $5,6,7,9,10,11,13,14,15$ e 17 seriam os mais influentes, como é mostrado nos gráficos da Figura 4.8 .
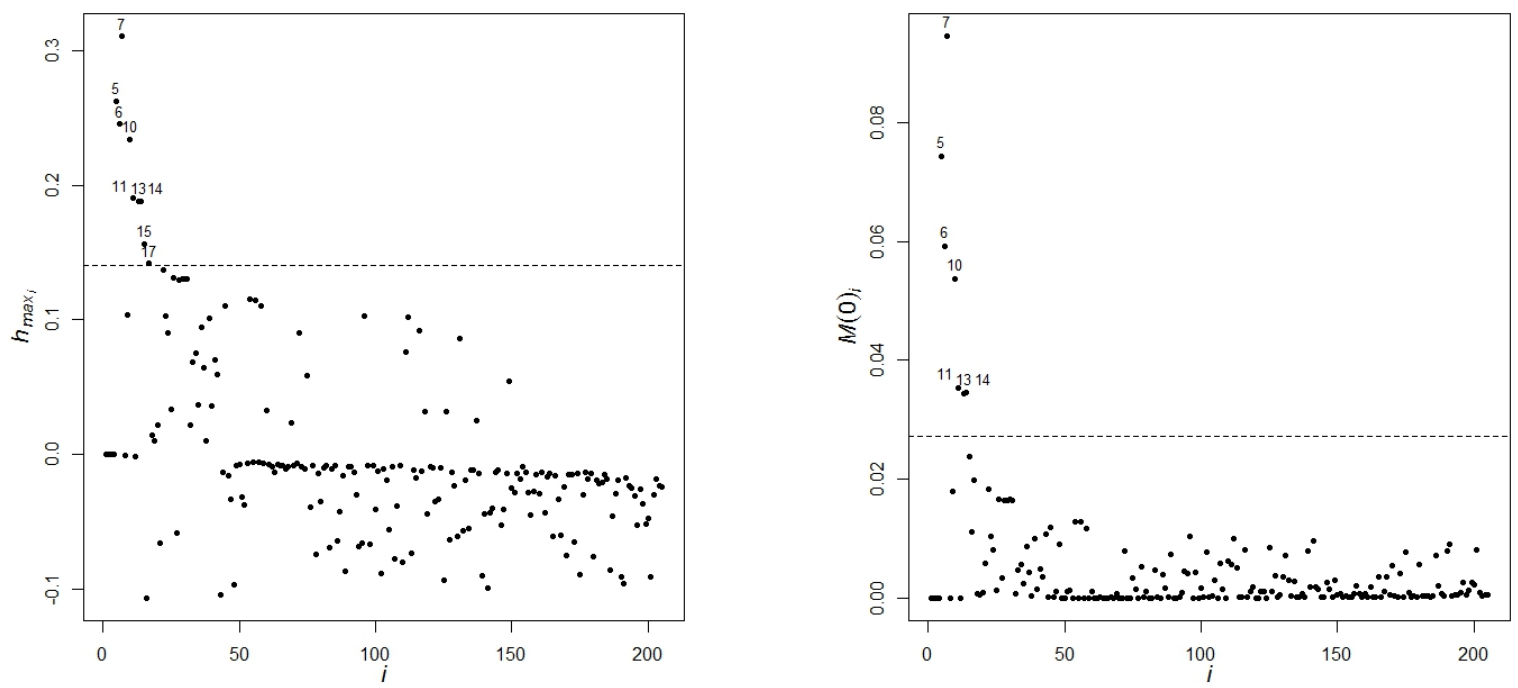

Figura 4.8: Gráficos de índices das medidas de influência local segundo o esquema de perturbação ponderada. Quadro esquerdo: Direção da máxima curvatura, $\boldsymbol{h}_{\max }$. Quadro direito: $M(0)_{i}$.

En resumo, segundo o análises de sensibilidade levado a cabo, o conjunto de casos potencialmente influentes é

$$
I=\{5,6,7,9,10,11,13,14,15,17,19,21,26,29,35,43,72,111,149\}
$$




\subsubsection{Impacto das observações influentes}

Para avaliar o impacto das observações em $I$, influentes, cada uma destas é retirada e o modelo proposto é ajustado novamente. Para medir o impacto da retirada sobre a estimativa do parâmetro $\psi_{i}$ é considerada a taxa de cambio relativa (rce):

$$
\operatorname{rce}\left(\psi_{i}\right)=100\left(\hat{\psi}_{i}-\hat{\psi}_{(i)}\right) / \hat{\psi}_{i}
$$

e o erro padrao estimado (rcse):

$$
\operatorname{rcse}\left(\psi_{i}\right)=100\left(\hat{s} e_{\psi_{i}}-\hat{s} e_{\psi_{(i)}}\right) / \hat{s} e_{\psi_{i}}
$$

Estas quantidades são mostradas na Tabela 4.5. Taxas de cambio consideráveis somente ocorrem na estimava do coeficiente de regressão $\beta_{4}$, associado com a covariável idade. Desde que esta covariável é retirada do modelo, porque resulta não significativa, o modelo proposto não parece ser sensitivo às observações influentes.

Tabela 4.5: Câmbios relativos nas estimativas (rce) e nos erros padrões estimados (rcse) dos parâmetros

\begin{tabular}{c|ccccccccccccccc} 
& \multicolumn{3}{|c}{$\alpha$} & \multicolumn{1}{c}{$\lambda$} & \multicolumn{3}{c}{$\beta_{1}$} & \multicolumn{3}{c}{$\beta_{2}$} & \multicolumn{3}{c}{$\beta_{3}$} & \multicolumn{3}{c}{$\beta_{4}$} & $\beta_{5}$ \\
Case & rce & rcse & rce & rcse & rce & rcse & rce & rcse & rce & rcse & rce & rcse & rce & rcse \\
\hline 5 & 0,5 & 1,1 & 0,5 & 2,1 & 1 & 0,3 & 0,7 & 0,6 & 10,8 & 4,5 & 27,4 & 1,7 & 4,6 & 0,5 \\
6 & 7,9 & 12,2 & 6,5 & 1,1 & 10,3 & 1,3 & 1,1 & 0,1 & 5,3 & 1,7 & 76,6 & 0,4 & 4,1 & 1,9 \\
7 & 8,2 & 13,4 & 9,6 & 1,4 & 6,1 & 4,3 & 0,9 & 0,5 & 9,1 & 0,6 & 77,8 & 1,6 & 6,2 & 0,8 \\
9 & 2,8 & 2,1 & 4,6 & 1,8 & 1,7 & 3,8 & 0,9 & 0,1 & 6 & 10,3 & 13,7 & 2,4 & 2,7 & 0,4 \\
10 & 5,8 & 9,3 & 3,5 & 0,4 & 0,8 & 1,0 & 1,0 & 0,1 & 1,0 & 1,5 & 26,3 & 0,9 & 16,8 & 0,6 \\
11 & 6,3 & 9,3 & 3,2 & 0,5 & 2,8 & 0,0 & 1,4 & 0,1 & 2,2 & 1,0 & 5,7 & 0,6 & 13,6 & 0,2 \\
13 & 3,7 & 5,8 & 0,8 & 0,3 & 1,8 & 0,4 & 2,0 & 0,1 & 2,1 & 0,8 & 25,6 & 0,6 & 11,4 & 0,1 \\
14 & 0,3 & 1,5 & 1,3 & 0,5 & 3,6 & 0,6 & 2,3 & 0,0 & 2,5 & 1,8 & 18,7 & 1,2 & 6,8 & 0,4 \\
15 & 1,8 & 2,7 & 2,6 & 0,3 & 8,0 & 3,6 & 2,8 & 0,4 & 0,1 & 0,0 & 73,7 & 1,5 & 9,2 & 0,9 \\
17 & 2,3 & 1,6 & 4,1 & 0,4 & 0,8 & 2,5 & 2,2 & 0,1 & 0,9 & 1,4 & 15,6 & 0,5 & 5,4 & 0,4 \\
19 & 2,6 & 1,6 & 3,8 & 1,7 & 5,5 & 5,5 & 1,4 & 0,3 & 0,3 & 2,5 & 30 & 11,7 & 3,8 & 0,8 \\
21 & 2,8 & 1,7 & 4,9 & 2,2 & 0,6 & 5,6 & 0,8 & 0,1 & 0,8 & 6,5 & 18,3 & 4,5 & 6,2 & 5,2 \\
26 & 0,5 & 0,6 & 2,9 & 0,1 & 0,0 & 3,0 & 4,3 & 1,9 & 0,7 & 0,2 & 21,1 & 0,3 & 4,5 & 0,7 \\
29 & 0,8 & 1,2 & 5,3 & 0,7 & 7,6 & 6,7 & 4,2 & 1,8 & 4,3 & 0,3 & 57,3 & 0,9 & 5,6 & 0,8 \\
35 & 3,3 & 1,9 & 5,0 & 3,2 & 2,2 & 3,6 & 2,0 & 6,5 & 5,4 & 8,3 & 4,5 & 0,5 & 2,7 & 0,1 \\
43 & 0,4 & 1,7 & 2,2 & 1,9 & 1,4 & 0,1 & 0,2 & 0,0 & 9,5 & 4,3 & 1,4 & 2,1 & 10,2 & 1,8 \\
72 & 1,2 & 0,3 & 0,4 & 2,0 & 1,4 & 0,0 & 3,5 & 2,4 & 2,4 & 1,6 & 37,0 & 2,5 & 5,8 & 1,0 \\
111 & 0,4 & 0,8 & 3,3 & 2,0 & 1,8 & 1,3 & 3,1 & 2,5 & 2,5 & 1,4 & 20,0 & 2,3 & 4,1 & 1,3 \\
149 & 2,1 & 3,7 & 9,8 & 2,3 & 4,4 & 4,0 & 3,0 & 2,8 & 4,8 & 1,4 & 23,8 & 4,1 & 1,9 & 2,0 \\
\hline
\end{tabular}




\subsubsection{Modelo final e bondade de ajuste}

Na Tabela 4.6 são listadas as estimativas dos parâmetros do modelo com fração de cura GELF, junto com estimativas dos seus erros padrões de estimação e intervalos de confiança do nivel 95\%. As covariáveis ulceration status, $\boldsymbol{X}_{2}$, espessura do tumor, $\boldsymbol{X}_{3}$, e sexo, $\boldsymbol{X}_{5}$, têm efeito significativo sobre a fração de cura. A proporção de pacientes curados é menor para pacientes con ulceração que para aqueles sem ela. A covariável grossura do tumor tem un efeito significativo na redução da proporção de curados. A proporção de mulheres curadas é maior que a correspondente aos homens.

Tabela 4.6: Estimativas de máxima verossimilhança dos parâmetros do modelo GELF.

\begin{tabular}{cccc}
\hline Parâmetro & Estimativa (est) & Erro padrão (se) & IC do $95 \%$ \\
\hline$\alpha$ & 2,9844 & 0,6133 & $(1,7824 ; 4,1864)$ \\
$\lambda$ & 0,2262 & 0,0851 & $(0,0595 ; 0,3930)$ \\
$\beta_{1}$ & 1,6351 & 0,4849 & $(0,6847 ; 2,5855)$ \\
$\beta_{2}$ & $-1,3947$ & 0,3152 & $(-2,0125 ;-0,7770)$ \\
$\beta_{3}$ & $-0,1174$ & 0,0337 & $(-0,1835 ;-0,0514)$ \\
$\beta_{5}$ & $-0,6014$ & 0,2725 & $(-1,1356 ;-0,0673)$ \\
\hline
\end{tabular}

A estimativa da proporção de curados para o $i$-ésimo individuo é dada por

$$
\hat{p}_{0 i}=\frac{\exp \left(1,6351-1,3947 x_{i 2}-0,1174 x_{i 3}-0,6014 x_{i 5}\right)}{1+\exp \left(1,6351-1,3947 x_{i 2}-0,1174 x_{i 3}-0,6014 x_{i 5}\right)},
$$

onde $x_{i 1}, x_{i 2}$ e $x_{i 5}$ são os valores correspondentes as covariáveis ulceração, grossura do tumor e sexo, respectivamente. Uma estimativa para a proporção de curados na população completa de indivíduos é dada por $\hat{p}_{0}=\frac{1}{205} \sum_{i=1}^{205} \hat{p}_{0 i}=0,5948$. A Figura 4.9 mostra a função de sobrevivência ajustada sobreposta à função de sobrevivencia empírica.

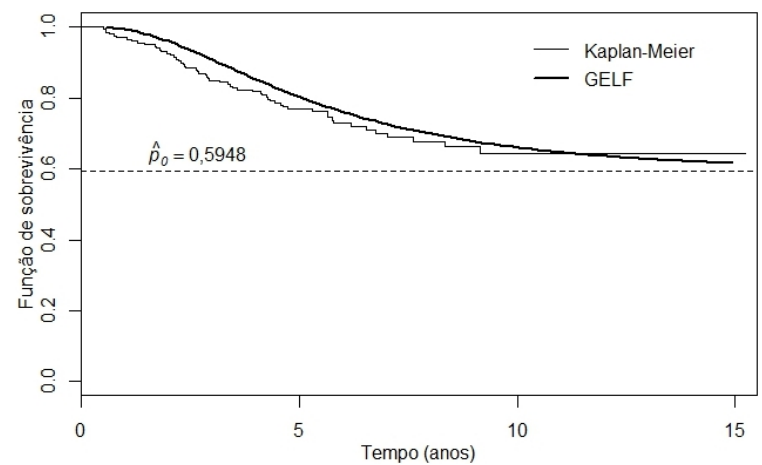

Figura 4.9: Estimativa de Kaplan-Meier da função de sobrevivência. 


\section{Capítulo 5}

\section{Conclusões e pesquisas futuras}

\subsection{Conclusões}

Neste estudo foi descrita a teoria dos modelos com fatores de risco latentes segundo esquemas de ativação, proposta por Cooner et al. (2007), e foram apresentados novos resultados a respeito. Considerando esta teoria foram deduzidos duas novas famílias de modelos de sobrevivência: a familia de distribuições exponencial-series de potências complementar, ESPC, e a família exponencial generalizada-series de potências com co-variáveis, EGSP. Para o modelo ESPC foi realizado um estudo de simulação considerando amostras de diferentes tamanhos para o caso particular exponencialbinomial, mostrando que as estimativas de máxima verossimilhança dos parâmetros e seus erros padrões de estimação, calculadas a partir da informação de Fisher observada, para amostras pequenas o viés resultou significativamente grande. Daí o uso da informacao de Fisher observada parece ser inapropriada, devido a isso foi considerado a metodologia do bootstrap para estimativa dos erros padrões das estimativas de máxima verossimilhança dos parâmetros do modelo ESPC. Além disso, o modelo proposto foi ajustado a um conjunto de dados de dados de sobrevivência. A família EGSP, que serve para modelar dados de sobrevivência com fração de cura, foi ajustada a um conjunto de dados de câncer. Além disso, para o modelo EGSP com covariáveis foram derivadas as curvaturas normais para se obterem as medidas de diagnósticos, baseado em vários esquemas de pertubação, de influência local e de influência global, bem como foram dadas medidas de diagnóstico baseados em deleção de casos (influência global). Um outro aspecto que foi abordado para o modelo EGSP foi a análise de resíduos sendo utilizado os quantis normalizados aleatorizados. 


\subsection{Pesquisas futuras}

Considerando os modelos para dados de sobrevivência com fração de cura, ou sem ela, dentro da teoria unificadora apresentada no Capítulo 2, as pesquisas propostas a ser desenvolvidas são.

1. Estudar o caso em que o tempo de vida seja discreto (Zhao \& Zhou, 2008).

2. Deduzir novas propriedades para o modelo proposto em Kim et al. (2011), pois, como foi mencionado no Capítulo 2 este modelo é similar ao modelo de Cooner et al. (2007).

3. Estudar a generalização dos modelos de Cooner et al. (2007) e Kim et al. (2011) para o caso de dois ou o mais tempos de vida.

4. Incorporar nos modelos estudados neste estudo outros tipos de censura.

5. Estudar propostas de modelos de sobrevivência de longa duração em que o tempo de vida nao seja uma variável aleatória estendida, ou seja, que não assuma o valor infinito.

6. Estudar a aplicação de outros tipos de curvaturas (vide Peng et al. (2007)) para realizar a análise de sensibilidade.

7. Considerar outras distribuições para os tempos de ativação, como a de Weibull exponenciada. 


\section{Referências Bibliográficas}

Aarset, M. V. (1985). The null distribution for a test of constant versus "bathtub" failure rate. Scandinavian Journal of Statistics, 12(1), 55-68.

Adamidis, K. \& Loukas, S. (1998). A lifetime distribution with decreasing failure rate. Statistics and Probability Letters, 39(1), 35-42.

Adamidis, K., Dimitrakopoulou, T. \& Loukas, S. (2005). On an extension of the exponential-geometric distribution. Statistics and Probability Letters, 73(3), 259 269.

Ash, R. B. (1972). Real analysis and probability. New York, Academic Press.

Balakrishnan, N. (2008). Over/under-dispersed Poisson distributions and processes. Conference presented at the 18th SINAPE - São Pedro, SP, Brasil.

Barlow, R. E., Marshall, A. W. \& Proschan, F. (1963). Properties of probability distributions with monotone hazard rate. The Annals of Mathematical Statistics, $\mathbf{3 4}(2), 375-389$.

Beckman, R. J., Nachtsheim, C. J. \& Cook, R. D. (1987). Diagnostics for mixed-model analysis of variance. Technometrics, 29(4), pp. 413-426.

Berkson, J. \& Gage, R. P. (1952). Survival cure for cancer patients following treatment. Journal of the American Statistical Association, 47(259), 501-515.

Boag, J. W. (1949). Maximum likelihood estimates of the proportion of patients cured by cancer therapy. Journal of the Royal Statistical Society, 11(1), 15-53.

Cancho, V. G. \& Bolfarine, H. (2001). Modeling the presence of immunes by using the exponentiated-Weibull model. Journal of Applied Statistics, 28(6), 659-671.

Cancho, V. G., Louzada-Neto, F. \& Barriga, G. D. C. (2011a). The Poisson-exponential lifetime distribution. Computational Statistics and Data Analysis, 55, 677-686. 
Cancho, V. G., Rodrigues, J. \& de Castro, M. (2011b). A flexible model for survival data with a cure rate: a Bayesian approach. Journal of Applied Statistics, 38, 57-70.

Cancho, V. G., de Castro, M. \& Dey, D. K. (2013). Long-term survival models with latent activation under a flexible family of distributions. Brazilian Journal of Probability and Statistics, 27(4), 585-600.

Chahkandi, M. \& Ganjali, M. (2009). On some lifetime distributions with decreasing failure rate. Computational Statistics and Data Analysis, 53(12), 4433-4440.

Chen, M.-H., Ibrahim, J. G. \& Sinha, D. (1999). A new Bayesian model for survival data with a surviving fraction. Journal of the American Statistical Association, 94(447), 909-919.

Chi, Y. Y. \& Ibrahim, J. G. (2007). Bayesian approaches to joint longitudinal and survival models accommodating both zero and nonzero cure fractions. Statistica Sinica, 17(2), 445-462.

Cook, R. D. (1977). Detection of infuential observations in linear regression. Technometrics, 19, 15-18.

Cook, R. D. (1986). Assessment of local influence. Journal of the Royal Statistical Society, Series B, 48, 133-169.

Cook, R. D. \& Weisberg, S. (1982). Residuals and Influence in Regression. Chapman \& Hall/CRC, Boca Raton, FL.

Cooner, F., Banerjee, S., Carlin, B. P. \& Sinha, D. (2007). Flexible cure rate modeling under latent activation schemes. Journal of the American Statistical Association, $\mathbf{1 0 2}(478), 560-572$.

Corless, R. M., Gonnet, G. H., Hare, D. E. G., Jeffrey, D. J. \& Knuth, D. E. (1996). On the lambert W function. Advances in Computational Mathematics, 5(1), 329-359.

Cox, D. R. \& Hinkley, D. V. (1974). Theoretical statistics. Chapman and Hall, London. de Castro, M., Cancho, V. G. \& Rodrigues, J. (2010). A note on a unified approach for cure rate models. Brazilian Journal of Probability and Statistics, 24(1), 100-103.

Dunn, P. K. \& Smyth, G. K. (1996). Randomized quantile residuals. Journal of Computational and Graphical Statistics, 5(3), pp. 236-244. 
Efron, B. \& Hinkley, D. V. (1978). Assessing the accuracy of the maximum likelihood estimator: Observed versus expected fisher information. Biometrika, 65(3), pp. $457-482$.

Efron, B. \& Tibshirani, R. (1986). Bootstrap methods for standard errors, confidence intervals, and other measures of statistical accuracy. Statistical Science, 1(1), 54-75.

Escobar, L. A. \& Meeker, William Q., J. (1992). Assessing influence in regression analysis with censored data. Biometrics, 48(2), pp. 507-528.

Farewell, V. T. (1977). A model for a binary variable with time-censored observations. Biometrika, 64(1), pp. 43-46.

Flores Delgado, J., Cancho, V. G. \& Chavez-Fuentes, R. (2012). The generalized exponential-power series cure rate model with covariates. Technical Report 385, Instituto de Ciências Matemáticas e de Computação. Universidade de São Paulo.

Flores Delgado, J., Borges, P., Cancho, V. G. \& Louzada-Neto, F. (2013). The complementary exponential power series distribution. Brazilian Journal of Probability and Statistics, 27(4), 401-617.

Gupta, R. D. \& Kundu, D. (1999). Theory and methods: Generalized exponential distributions. Australian and New Zealand Journal of Statistics, 41(2), 173-188.

Gupta, R. D. \& Kundu, D. (2003). Discriminating between weibull and generalized exponential distributions. Computational Statistics and Data Analysis, 43(2), 179 196.

Hoggart, C. J. \& Griffin, J. E. (2001). A Bayesian partition model for customer attrition. In E. I. George, editor, Bayesian Methods with Applications to Science, Policy, and Official Statistics (Selected Papers from ISBA 2000 - Creta, Greece), pages 61-70. International Society for Bayesian Analysis.

Ibrahim, J. G., Chen, M.-H. \& Sinha, D. (2001). Bayesian Survival Analysis. Springer, New York, NY.

James, B. R. (1981). Probabilidade: um curso em nível intermediário. Projeto Euclides.

Johnson, L. N., Kemp, A. W. \& Kotz, S. (2005). Univariate Discrete Distributions. Wiley, New York, NY, third edition.

Kannan, N., Kundu, D., Nair, P. \& Tripathi, R. C. (2010). The generalized exponential cure rate model with covariates. Journal of Applied Statistics, 37(10), 1625-1636. 
Kaplan, E. L. \& Meier, P. (1958). Nonparametric estimation from incomplete observations. Journal of the American Statistical Association, 53(282), pp. 457481.

Kim, S., Chen, M.-H. \& Dey, D. (2011). A new threshold regression model for survival data with a cure fraction. Lifetime Data Analysis, 17, 101-122. 10.1007/s10985010-9166-9.

Kus, C. (2007). A new lifetime distribution. Computational Statistics and Data Analysis, 51(9), 4497-4509.

Lawless, J. F. (2003a). Statistical Models and Methods for Lifetime Data. Wiley, New York, NY, first edition.

Lawless, J. F. (2003b). Statistical Models and Methods for Lifetime Data. Wiley, New York, NY, second edition.

Li, A.-P., Chen, Z.-X. \& Xie, F.-C. (2012). Diagnostic analysis for heterogeneous log-Birnbaum-Saunders regression models. Statistics and Probability Letters, 82(9), 1690-1698.

Li, C.-S., Taylor, J. M. \& Sy, J. P. (2001). Identifiability of cure models. Statistics and Probability Letters, 54, 389-395.

Maller, R. A. \& Zhou, S. (1992). Estimating the proportion of immunes in a censored sample. Biometrika, 79(4), pp. 731-739.

Maller, R. A. \& Zhou, X. (1996). Survival Analysis with Long-Term Survivors. Wiley, New York, NY.

Marsaglia, G., Tsang, W. W. \& Wang, J. (2003). Evaluating kolmogorov's distribution. Journal of Statistical Software, 8(18), 1-4.

Mendenhall, W. \& Sincich, T. (1995). Probabilidad y Estadística para ingeniería y ciencias. Prentice-Hall Hispanoamericana, S.A., México, fourth edition.

Morais, A. L. \& Barreto-Souza, W. (2011). A compound class of weibull and power series distributions. Computational Statistics and Data Analysis, 55(3), 1410-1425.

Peng, L., Huafeir, S. \& Lin, J. (2007). The geometric structure of the pareto distribution. Boletín de la Asociación Matemática Venezolana, XIV(1-2), 5-13.

Pike, M. C. (1966). A method of analysis of a certain class of experiments in carcinogenesis. Biometrics, 22(1), pp. 142-161. 
Poon, W.-Y. \& Poon, Y. S. (1999). Conformal normal curvature and assessment of local influence. Journal of the Royal Statistical Society: Series B (Statistical Methodology), 61(4), 51-61. 10.1111/1467-9868.00162.

R Development Core Team (2011). R: A Language and Environment for Statistical Computing. R Foundation for Statistical Computing, Vienna, Austria. ISBN 3900051-07-0.

Rigby, R. A. \& Stasinopoulos, D. M. (2005). Generalized additive models for location, scale and shape. Journal of the Royal Statistical Society. Series C (Applied Statistics), 54(3), pp. 507-554.

Rodrigues, J., Cancho, G. V., de Castro M. \& Louzada-Neto, F. (2009a). On the unification of the long-term survival models. Statistics and Probability Letters, 79, $753-759$.

Rodrigues, J., de Castro, M., Cancho, V. G. \& Balakrishnan, N. (2009b). Com-poisson cure rate survival models and an applications to a cutaneous melanoma data. Journal of Statistical Planning and Inference, 139, 3605-3611.

Rodrigues, J., de Castro, M., Balakrishnan, N. \& Cancho, V. G. (2010). Destructive weighted poisson cure rate models. Lifetime Data Analysis, 17(3), 333-346.

Rudin, W. (1976). Principles of mathematical analysis. McGraw-Hill, Inc., third edition.

Scheike, T. (2009). timereg package. With contributions from Torben Martinussen and Jeremy Silver. R package version 1.1-6.

Tahmasbi, R. \& Rezaei, S. (2008). A two-parameter lifetime distribution with decreasing failure rate. Computational Statistics and Data Analysis, 52, 3889-3901.

Tsodikov, A. (1998). A proportional hazards model taking account of long-term survivors. Biometrics, 54(1), 1508-1516.

Tsodikov, A. D., Ibrahim, J. G. \& Yakovlev, A. Y. (2003). Estimating cure rates from survival data: an alternative to two-component mixture models. Journal of the American Statistical Association, 98(464), 1063-1078.

Yakovlev, A., Yu, A. B., Bardou, V.-J., Fourquet, A., Hoang, T., Rochefodiere, A. \& Tsodikov, A. D. (1993). A simple stochastics model of tumor recurrence an its aplications to data on premenopausal breast cancer. In S. F. de Biométrie, editor, Biometrie et Analyse de Donnes Spatio-Temporelles No 12, B, pages 33-82, France. 
Yakovlev, A. Y. \& Tsodikov, A. D. (1993). A stochastic-model of hormesis. Mathematical Biosciences, 116(2), 197-219.

Zhao, X. \& Zhou, X. (2008). Discrete-time survival models with long-term survivors. Statistics in Medicine, 27(8), 1261-1281.

Zhu, H.-T. \& Lee, S.-Y. (2001). Local influence for incomplete-data models. Journal of the Royal Statistical Society. Series B (Statistical Methodology), 63(1), pp. 111-126. 